\title{
Sistema de caracterização elétrica de dispositivos emissores de campo
}

Dissertação apresentada à Escola Politécnica da Universidade de São Paulo para obtenção do Título de Mestre em Engenharia Elétrica.

Área de Concentração:

Sistemas Eletrônicos

Orientador:

Prof. Dr. Francisco Javier

Ramirez Fernandes 
AUTORIZO A DIVULGAÇÃO E REPRODUÇÃO TOTAL OU PARCIAL DESTE TRABALHO, POR QUALQUER MEIO CONVENCIONAL OU ELETRONICO, PARA FINS DE ESTUDO E PESQUISA, DESDE QUE CITADA A FONTE

FICHA CATALOGRÁFICA

Kopelvski, Maycon Max

Sistema de caracterização elétrica de dispositivos emissores de campo / M.M. Kopelvski. -- São Paulo, 2007.

$98 \mathrm{p}$.

Dissertação (Mestrado) - Escola Politécnica da Universidade de São Paulo. Departamento de Engenharia de Sistemas Eletrônicos.

1.Dispositivos emissores de campo elétrico 2.Espectrometria de massa 3.Instrumentação virtual I.Universidade de São Paulo. Escola Politécnica. Departamento de Engenharia de Sistemas Eletrônicos II.t. 
KOPELVSKI, MAYCON M. Sistema de caracterização elétrica de dispositivos emissores de campo. 2007. 98 f. Dissertação (Mestrado) - Escola Politécnica - Universidade de São Paulo - São Paulo, 2007.

\section{ERRATA}

$\begin{array}{llll}\text { Folha } & \text { Linha } & \text { Onde se lê } & \text { Leia-se } \\ 14 & 6 & \text { FE } & \text { FED } \\ 29 & 19 & \text { Assembler } & \text { Assembly } \\ 33 & 8 & \text { a través } & \text { através } \\ 36 & 6 & \text { Assembler } & \text { Assembly } \\ 37 & 13 & \text { Tensão } & \text { tensão } \\ 39 & 1 & \text { FOTO AC. } & \text { Foto acoplador } \\ 39 & 15 & \text { display } & \\ 40 & 2 & \text { Fig.4.7 } & \text { Fig.4.6 } \\ 40 & 9 & \text { calibração } & \text { calibração (Fig. 4.7) } \\ 41 & 3 & \text { seguir } & \text { seguir (Fig.4.8) } \\ 43 & 8 & \text { a fonte } & \text { o hardware } \\ 49 & 2 & \text { medido... } & \text { medido. } \\ 49 & 29 & \text { amostrados } & \text { amostrados e } \\ & & \text { armazenados } & \text { armazenados } \\ 52 & 8,12,14 & \text { casa } & \text { casas } \\ 55 & 10 & \text { GSIM } & \text { GSIM (Grupo de Sensores } \\ & & & \text { Integráveis e Microssistemas) } \\ 55 & 29 & \text { Figura } 4.11 & \text { Figura 4.10 } \\ 56 & 12 & \text { resistência } & \text { resistência, equivalente a } \\ & & \text { equivalente } & \\ 56 & 13 & \text { Figura 4.12 } & \text { Figura 4.11 } \\ 57 & 9 & \text { Figura 4.14 } & \text { Figura 4.12 } \\ 59 & 14 & \text { Figura 4.25 } & \text { Figura 4.23 } \\ 62 & 2 & \text { Figura 4.17 } & \text { Figura 4.16 } \\ 67 & 8 & \text { Fig.4.31 } & \text { Fig.4.29 } \\ & & & \end{array}$




\section{FOLHA DE APROVAÇÃO}

Maycon Max Kopelvski

Sistema de caracterização elétrica de dispositivos emissores de campo

Dissertação apresentada à Escola Politécnica da Universidade de São Paulo para obtenção do Título de Mestre em Engenharia Elétrica.

Área de Concentração:

Sistemas Eletrônicos

Aprovado em: l /

Banca Examinadora:

Prof. Dr.:

Instituição: Assinatura:

Prof. Dr.:

Instituição: Assinatura:

Prof. Dr.:

Instituição: Assinatura: 


\section{DEDICATÓRIA}

Aos pilares que nunca me deixaram cair - Thais, minha esposa, Marllon e Magnon, meus irmãos e Marco Antonio e Maria Jose, meus pais - com amor, admiração e gratidão por sua compreensão, carinho, presença e incansável apoio e torcida ao longo do período de elaboração deste trabalho. 


\section{AGRADECIMENTOS}

Agradeço a Deus por mais essa graça concedida.

Ao Prof. Dr. Francisco Javier Ramirez Fernandes, que nos anos de orientação e convivência constante, muito me ensinou, contribuindo para meu crescimento cientifico e intelectual.

Aos integrantes do Grupo de Sensores Integráveis e Microssistemas em especial - ao Henrique E. M. Peres, pelo fundamental apoio, abraçando a causa e empenhando um esforço muitas vezes além do necessário para que obtivéssemos sucesso no desenvolvimento desse projeto. A Elizabete Galeazzo, pelas sugestões ao revisar os textos da dissertação e congressos, além de que, juntamente com o Michel O. S. Dantas, exerceram papel importantíssimo durante as etapas de especificação do sistema, e ensaios, sempre transmitindo inúmeros conhecimentos relacionados aos FED's. E ao Maurício O. P. Lisboa pelo apoio técnico relacionado ao desenvolvimento do hardware desse trabalho.

Ao Mauro Braga, M. D. Pilar H. Falla, Thiago H. D. Faria, Wesley Becari, que sempre ao nos encontrar no Laboratório de Microeletrônica, sempre tinham alguma experiência e conhecimento para compartilhar.

À Escola Politécnica da Universidade de São Paulo, pela oportunidade de realização do curso de mestrado.

Ao senhor Salvador Pinillos Gimenez pela confiança depositada antes mesmo de me tornar um aluno de pós-graduação.

Ao professor Aderbal de Arruda Penteado Jr., que da sua maneira e sempre com uma presteza admirável, me apoiou e incentivou para que pudesse me desenvolver cada vez mais meus conhecimentos acadêmicos.

Aos professores Mário César G. Ramos e Antonio José do Couto Pitta pelo apoio incondicional e por acreditarem na superação dos obstáculos e na conquista desse objetivo.

Aos meus amigos de graduação, onde, sem exceção, me apoiaram e confiaram que estariam sendo bem representados e que não seria medido esforços para que essa conquista fosse alcançada.

Enfim, a todos os amigos, alunos e professores, que de alguma forma contribuíram para o desenvolvimento desse trabalho. 
"Nunca ande pelo caminho traçado, pois ele conduz somente até onde os outros foram..." Alexandre Graham Bell 


\section{RESUMO}

KOPELVSKI, MAYCON M. Sistema de caracterização elétrica de dispositivos emissores de campo. 2007. 98 f. Dissertação (Mestrado) - Escola Politécnica - Universidade de São Paulo - São Paulo, 2007.

Neste trabalho é apresentado o desenvolvimento de um sistema de ensaios elétricos de dispositivos de emissão de campo para que, a partir desses ensaios, possam ser extraídas as características elétricas desses dispositivos. O sistema é composto por hardware e software dedicados e pode ser controlado local ou remotamente. O hardware inclui uma fonte de alta tensão gerenciada por um sistema microcontrolado. Para programação do microcontrolador, foi utilizado um ambiente de programação disponibilizado pelo próprio fabricante do microcontrolador. Nesse desenvolvimento foram empregados periféricos de entrada e saída do microcontrolador, tais como: leitura de teclado, manipulação de USART, ajuste do nível de saída da fonte e conversores analógicos digitais. No microcontrolador foram implantadas rotinas de configuração, personalização e varredura do display, além de envio e recebimento de informações com um computador. Para o computador foi elaborado um programa dedicado para a manipulação do sistema de ensaio utilizando o conceito de instrumentação virtual, que permite escolher o tipo de ensaio elétrico, armazenar as leituras dos ensaios e a visualização "on-line" do andamento do ensaio através de diversos gráficos disponíveis no programa, inclusive o gráfico de Fowler-Nordheim, adequado para o estudo de dispositivos de emissão de campo.

Palavras Chaves: emissão de campo, caracterização elétrica, micropontas de silício, espectrometria de massa, fonte de alta tensão, traçador de curvas, instrumentação virtual, analisador de parâmetros. 


\begin{abstract}
KOPELVSKI, MAYCON M. Field emission devices electrical characteristics trial system. 2007. 98 f. Dissertation (Masters) - Escola Politécnica - Universidade de São Paulo - São Paulo, 2007.

At this work is presented the development of a field emission devices trial system to render possible to obtain the electrical characteristics of the field emission devices. Here are shown some results taken from some trials. During the development of the trial system, it was used at the programming microcontroller stage, the environment of programming supplied by the manufacturer of the microcontroller. At this development, peripheral of input and output from the microcontroller, like, keyboard reading, USART manipulation, SPI manipulation and analogic to digital converters were used. At the microcontroller were implanted routines of configuration, customing and display sweeping, besides the transmission and the receiving of instructions came from the computer. For computer, a program was elaborated dedicated for manipulation of the trials system applying the virtual instrumentation concept, storing readings of the trials as well as the visualization "on-line" of the course of the trial through available graphs in the program. As an important result of this work has the establishment of a system for trial of field emission devices controlled on place or remotely, system that is composed by hardware and software in which were made several trials with acquisition and data manipulation and the presentation of received information.
\end{abstract}

Keywords: field emission, electrical characterization, silicon microtips, mass spectrometry, high voltage source, curve tracer, virtual instrumentation, parameter analyzer. 


\section{LISTA DE FIGURAS}

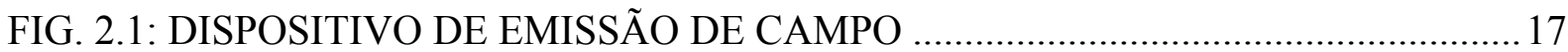

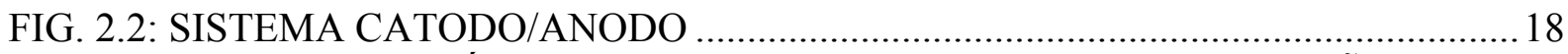

FIG. 2.3: EXEMPLO GENÉRICO DE FERRAMENTA DE INSTRUMENTAÇÃO

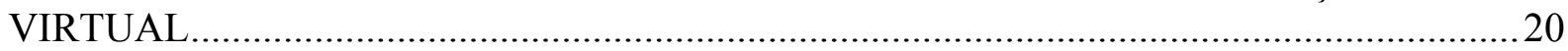

FIG. 2.4: ESQUEMA ILUSTRATIVO DE UM DISPOSITIVO DE EMISSÃO

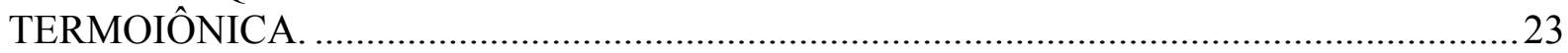

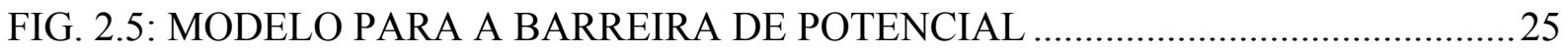

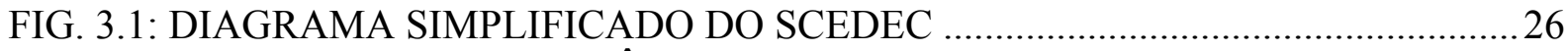

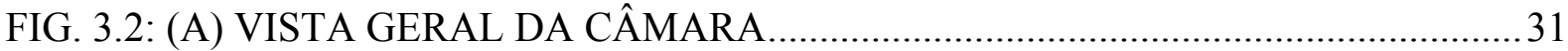

FIG. 3.3: ETAPAS A SEREM DESENVOLVIDAS PARA A REALIZAÇÃO DESTE

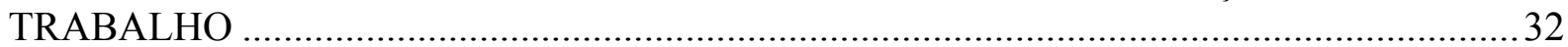

FIG. 3.4: SISTEMA DE CARACTERIZAÇÃO DOS DISPOSITIVOS FE ............................ 34

FIG. 3.5: METODOLOGIA DE DESENVOLVIMENTO ADOTADA PARA O PROJETO 34

FIG. 4.1: DIAGRAMA EM BLOCOS DO HARDWARE DESENVOLVIDO........................36

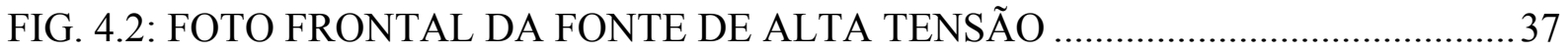

FIG. 4.3: FOTO SUPERIOR DA FONTE DE ALTA TENSÃO …...................................... 38

FIG. 4.4: REFERÊNCIAS DOS BLOCOS DE TENSÃO PRIMÁRIA ................................. 39

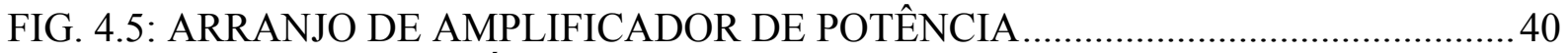

FIG. 4.6: CIRCUITO DO MÓDULO DE ALTA TENSÃO ...................................................... 40

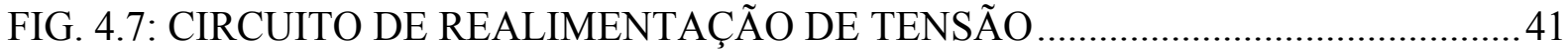

FIG. 4.8: CIRCUITO DE REALIMENTAÇÃO DE CORRENTE ......................................... 41

FIG. 4.9: "SPLASH SCREEN" DO PROGRAMA HIGHVOLT CONTROLLER ...................... 43

FIG. 4.10: ESQUEMA DE LIGAÇÃO DO CIRCUITO DE CALIBRAÇÃO .........................56

FIG. 4.11: ESQUEMA DE LIGAÇÃO DA RESISTÊNCIA DE $1 \mathrm{G} \Omega$....................................56

FIG. 4.12: ESQUEMA DE LIGAÇÃO DA RESISTÊNCIA DE 200M $\Omega$................................57

FIG. 4.13: PONTOS DE MEDIÇÃO DA RESISTÊNCIA DE 20M $\Omega$...................................... 58

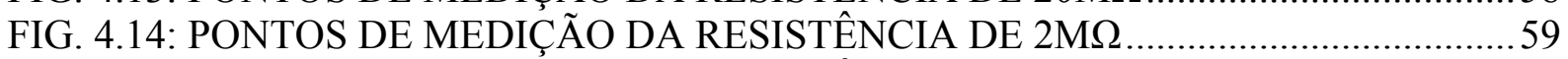

FIG. 4.15: PONTOS DE MEDIÇÃO DA RESISTÊNCIA DE 200K $\Omega$....................................59

FIG. 4.16: GRÁFICO DE CORRENTE X TENSÃO (500NA - RESISTOR DE 2G $\Omega$ ) ........60

FIG. 4.17: GRÁFICO DE CORRENTE X TENSÃO (5MA - RESISTOR DE 200M 2 ) .......60

FIG. 4.18: GRÁFICO DE CORRENTE X TENSÃO (50MA - RESISTOR DE 20M $\Omega$ ) .......61

FIG. 4.19: GRÁFICO DE CORRENTE X TENSÃO (500MA - RESISTOR DE 2M $\Omega$ ) ......6 61

FIG. 4.20: GRÁFICO DE CORRENTE X TENSÃO (5MA - RESISTOR DE 200K $\Omega$ )........6 61

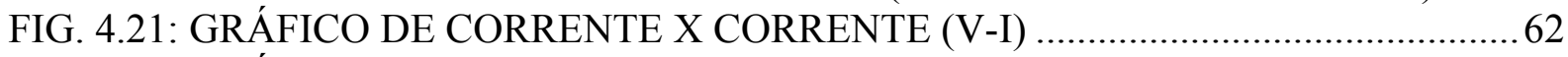

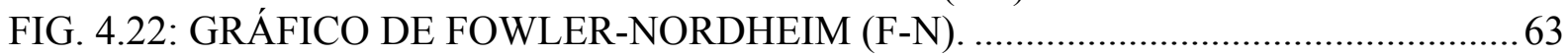

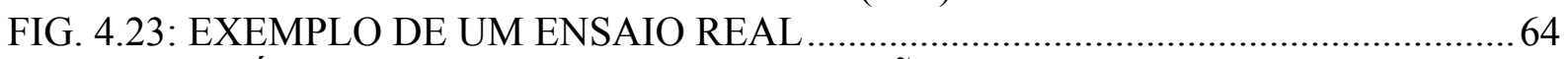

FIG. 4.24: GRÁFICO COMPARATIVO DE MEDIÇÕES (ESCALA DE 500NA)................65

FIG. 4.25: GRÁFICO COMPARATIVO DE MEDIÇÕES (ESCALA DE $5 \mu \mathrm{A})$. ....................6 65

FIG. 4.26: GRÁFICO COMPARATIVO DE MEDIÇÕES (ESCALA DE 50 $\mu$ A) ................66

FIG. 4.27: GRÁFICO COMPARATIVO DE MEDIÇÕES (ESCALA DE 500 $\mu$ A) ...............66

FIG. 4.28: GRÁFICO COMPARATIVO DE MEDIÇÕES (ESCALA DE 5MA)..................67

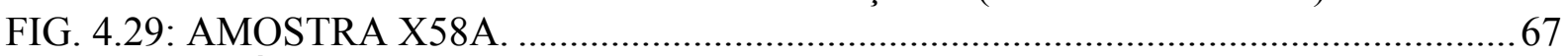

FIG. 4.30: GRÁFICO DOS ENSAIOS COM O EQUIPAMENTO KEITHLEY....................6 68

FIG. 4.31: GRÁFICO DOS ENSAIOS COM O SCEDEC......................................................6

FIG. 4.32: GRÁFICO COMPARATIVO DE MEDIÇÕES KEITHLEY VERSUS SCEDEC 69

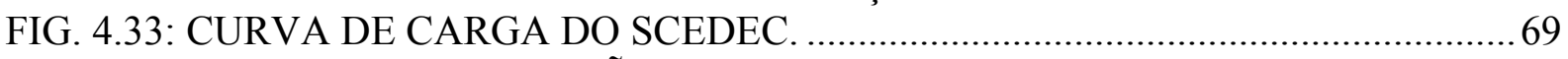

FIG. 4.34: CURVA DE REGULAÇÃO DO SCEDEC ........................................................ 70 


\section{LISTA DE TABELAS}

TABELA 3.1 COMPONENTES UTILIZADOS NO TRABALHO 28

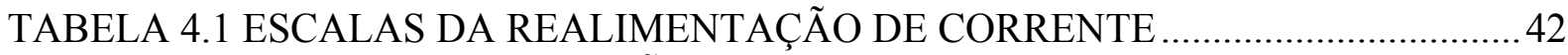

TABELA 4.2 ESCALAS DE MEDIÇÃO DE CORRENTE .............................................. 49

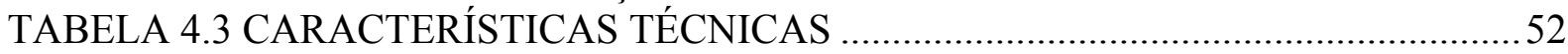




\section{LISTA DE ABREVIATURAS E SIGLAS}

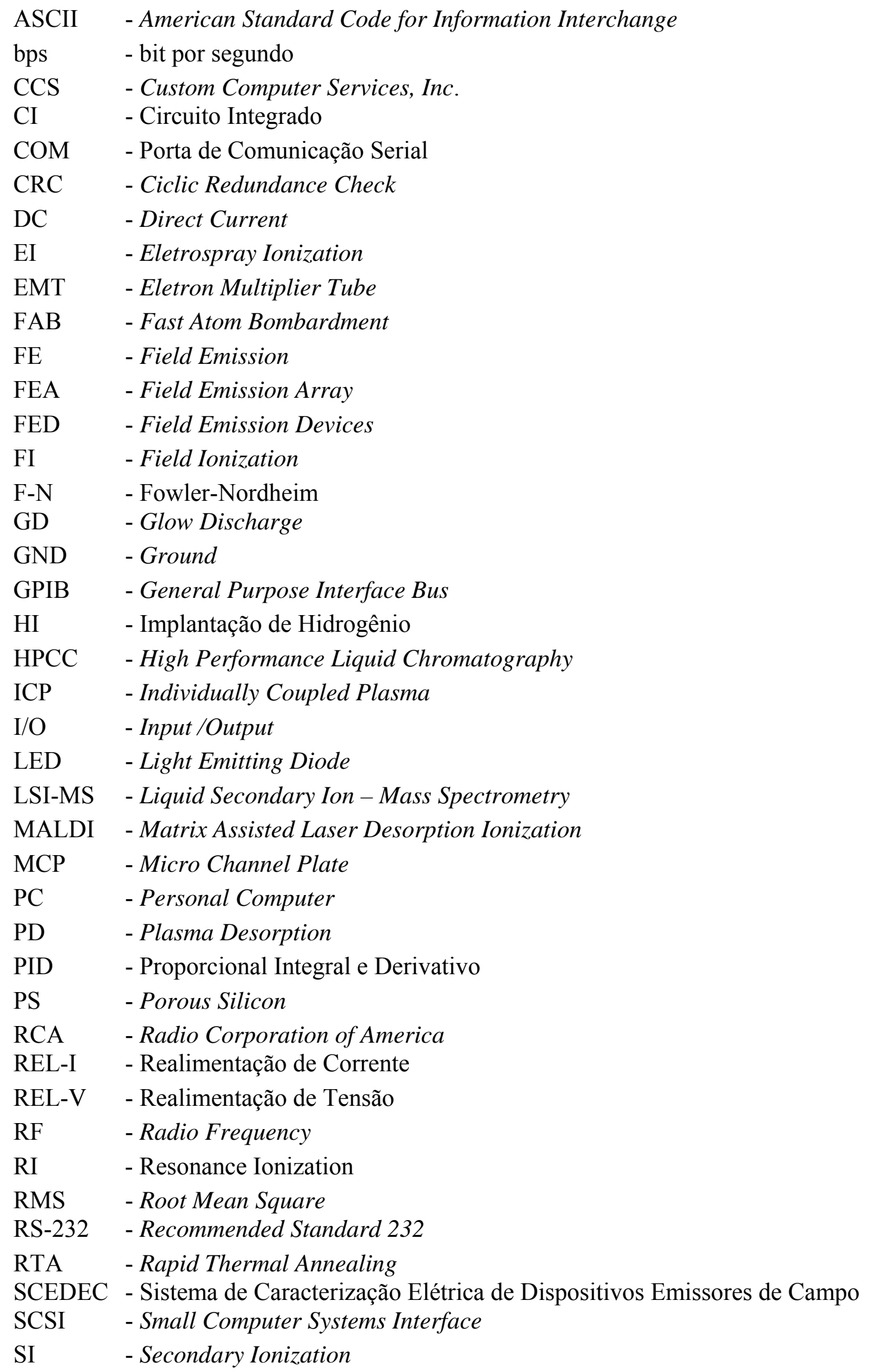




$\begin{array}{ll}\text { SPI } & \text { - Serial Peripheral Interface } \\ \text { SS } & \text { - Spark Source } \\ \text { TI } & \text { - Thermal Ionization } \\ \text { TOF } & \text { - Time Of Flight } \\ \text { USART } & \text { - Universal Synchronous Asynchronous Receiver Transmitter } \\ \text { USB } & \text { - Universal Serial Bus } \\ \text { VME } & \text { - Vacuum Micro Electronics }\end{array}$




\section{LISTA DE SÍMBOLOS}

$$
\begin{array}{ll}
\Phi & \text { - função trabalho } \\
\omega & \text { - freqüência angular } \\
\mu \mathrm{C} & \text { - microcontrolador } \\
\mathrm{m} / \mathrm{z} & \text { - relação massa / carga de íon } \\
\mathrm{r} & \text { - raio da microponta }
\end{array}
$$


RESUMO

\section{SUMÁRIO}

ABSTRACT

LISTA DE FIGURAS

LISTA DE TABELAS

LISTA DE ABREVIATURAS E SIGLAS

LISTA DE SÍMBOLOS

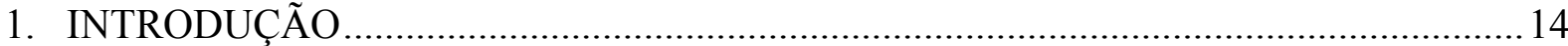

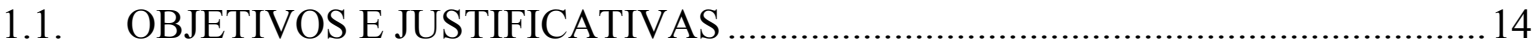

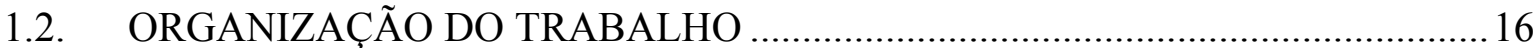

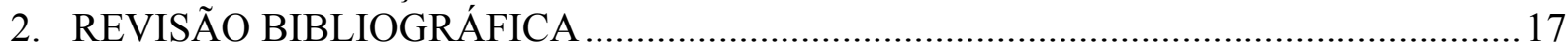

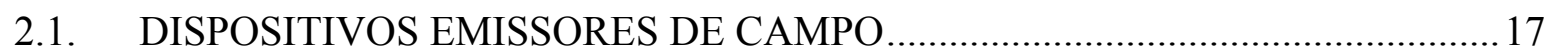

2.2. INSTRUMENTAÇÃO VIRTUAL .................................................................... 19

2.2.1 PARÂMETROS QUE DESCREVEM A INSTRUMENTAÇÃO ...................21

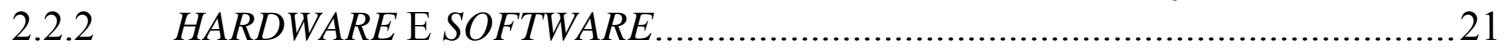

2.2.3 DESENVOLVIMENTO DE UMA INSTRUMENTAÇÃO DEDICADA

PARA CARACTERIZAÇÃO DE DISPOSITIVOS EMISSORES DE CAMPO ............22

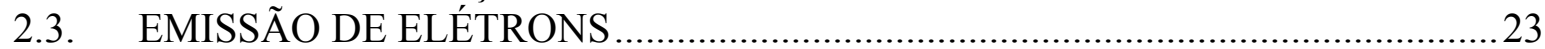

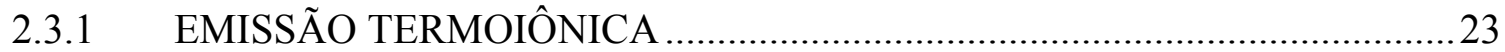

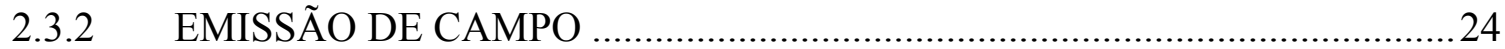

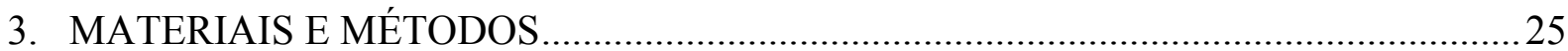

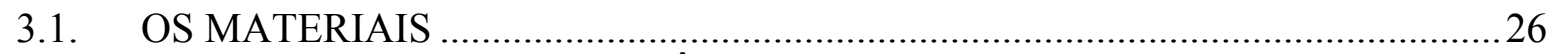

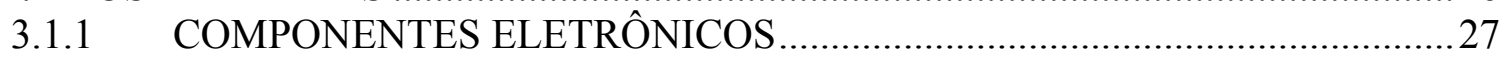

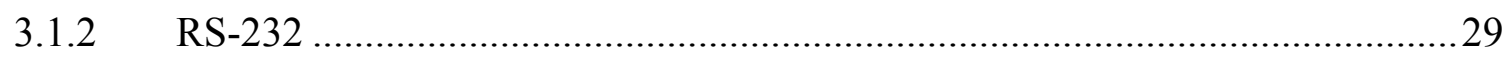

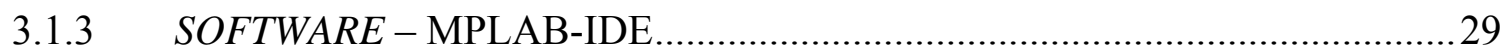

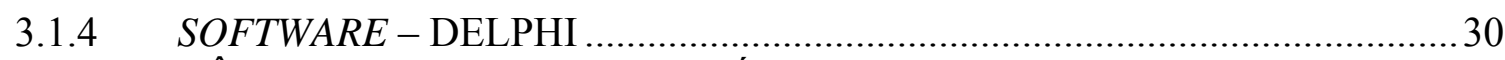

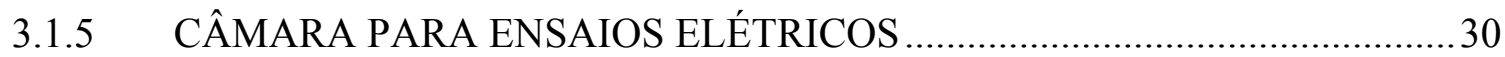

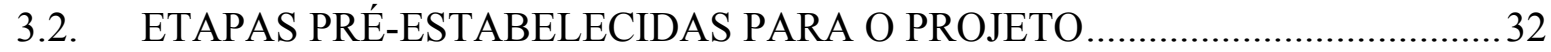

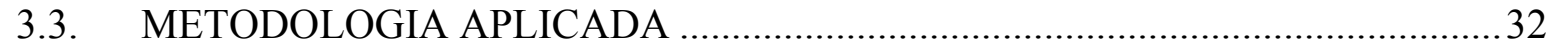

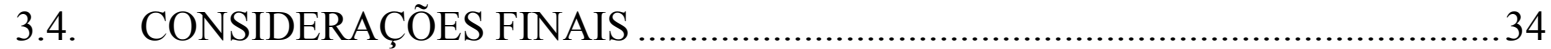

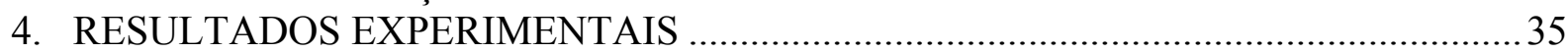

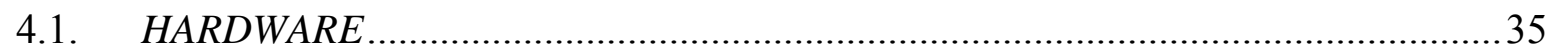

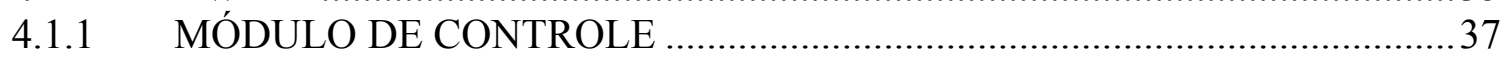

4.1.2 SOFTWARE - HIGHVOLT CONTROLLER …............................................. 42

4.1.3 PROTOCOLO DE COMUNICAÇÃO PC - FONTE ........................................ 44

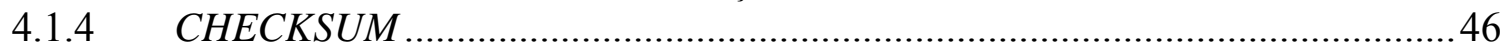

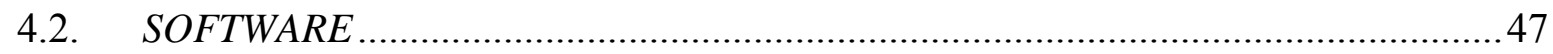

4.2.1 ESTRUTURA DO HIGHVOLT CONTROLLER …......................................47

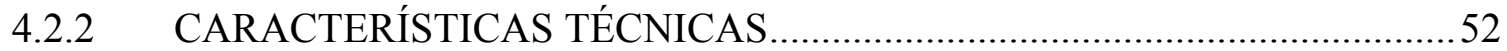

4.2.3 PROTOCOLO DE COMUNICAÇÃO FONTE - PC ........................................52

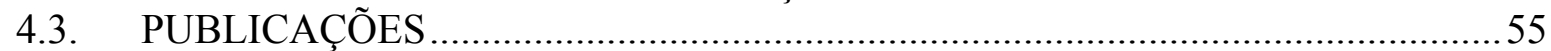

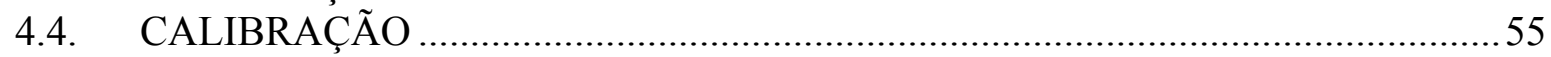

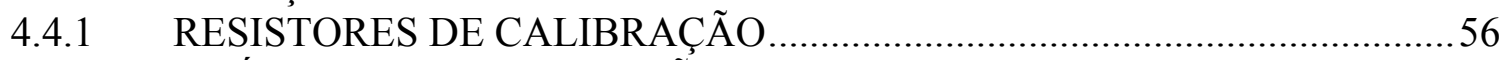

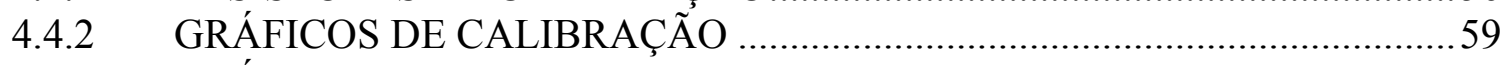

4.4.3 GRÁFICOS OBTIDOS EM ENSAIOS COM DISPOSITIVOS FE .................62

4.5. COMPARAÇÃO DE INSTRUMENTOS VERSUS SCEDEC COM CARGA RESISTIVA 


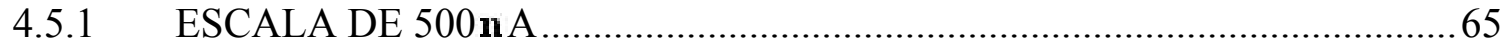

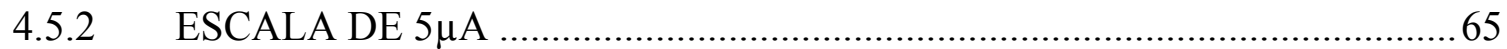

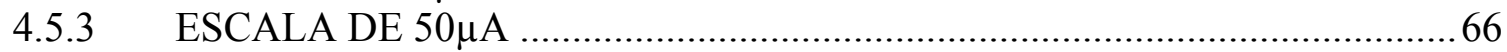

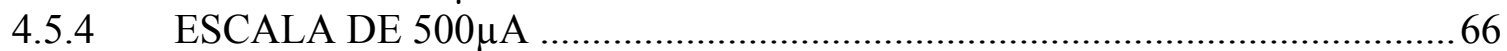

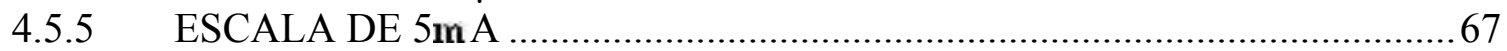

4.6. COMPARAÇÃO DE INSTRUMENTOS VERSUS SCEDEC COM FED'S NA

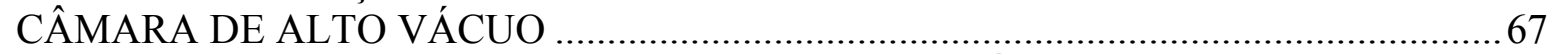

4.6.1 ENSAIO COM O VOLTIMETRO E AMPERÍMETRO KEITHLEY .............68

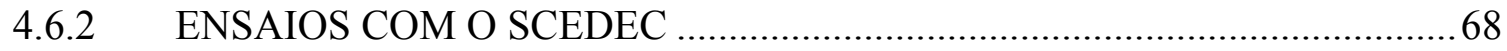

4.6.3 COMPARATIVO DAS MEDIÇÕES KEITHLEY VERSUS SCEDEC .........69

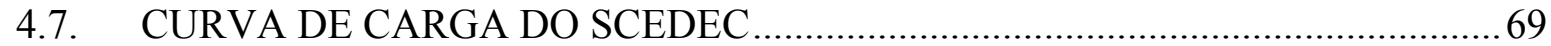

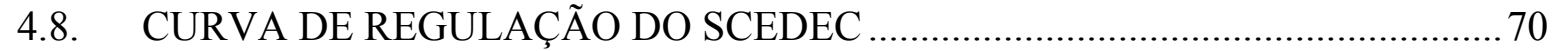

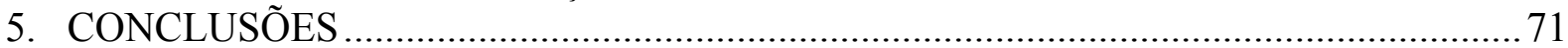

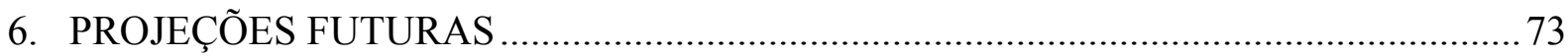

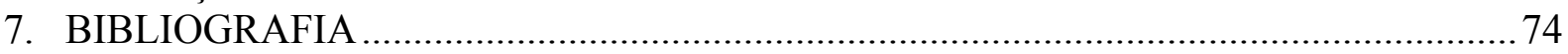

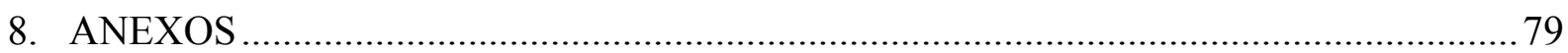

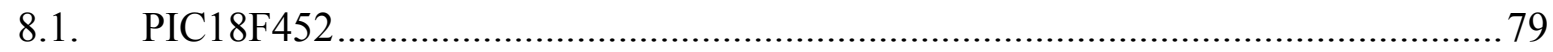

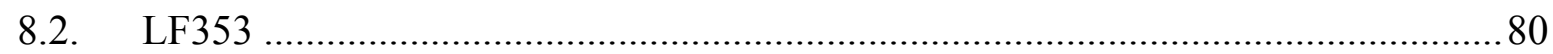

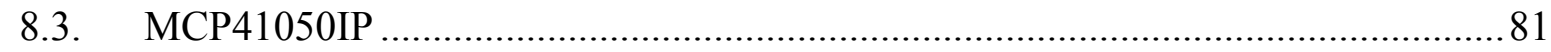

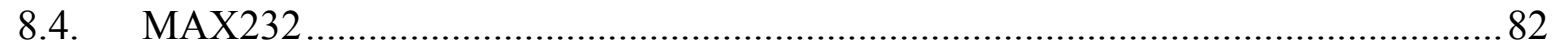

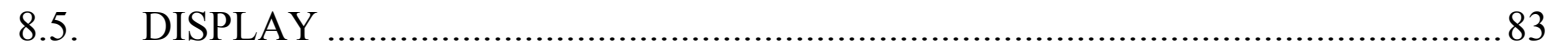

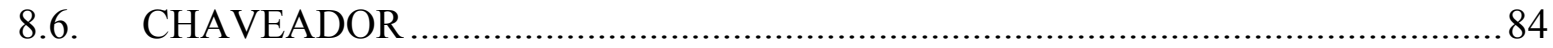

8.7. RAIZ DO PROGRAMA DO MICROCONTROLADOR …....................................85

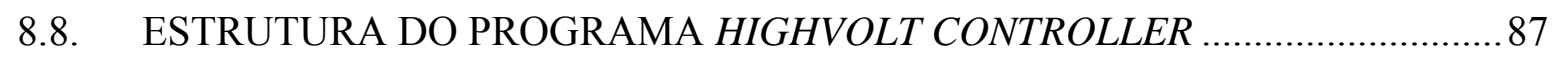

8.9. CALCULO DO CHECKSUM DO PACOTE DE DADOS ..................................... 89

8.10. RAIZ DO PROGRAMA HIGHVOLT CONTROLLER ................................. 90 


\section{INTRODUÇÃO}

\subsection{OBJETIVOS E JUSTIFICATIVAS}

Este trabalho apresenta o desenvolvimento de um sistema de baixo custo, com alta confiabilidade e precisão, que apresenta integração entre software e hardware num contexto de instrumentação virtual, focado para aplicação no processo de caracterização elétrica de dispositivos de emissão de campo (FE - "field emission devices"). Esta caracterização concentra-se essencialmente em extrair as características I-V dos dispositivos em diversas condições de operação.

FE são dispositivos constituídos por dois eletrodos que podem ser integrados ou não. Um eletrodo é denominado catodo e o outro anodo com o objetivo de ser produzido elevado campo elétrico com potenciais elétricos relativamente baixos

Dispositivos emissores de campo são aplicados em diversas áreas, como:

- Displays planos de baixa voltagem com alta resolução e brilho (FURSEY,2003) (XU,2005) (MAMMANA,2000) (TALIN,2001) (DEBSKI,2001) (CHUNG,2004) (LEE,2000) (SILVA,2004);

- Sistemas optoeletrônicos de elevada resolução (FURSEY,2003) (ZUUK,2004) (HIRAYAMA,2002) (GÖLZHÄUSER,1999) (BALE,2002) (MITA,2005);

- Emprego em dispositivos de microondas (FURSEY,2003) (JENSEN,2001) (LI,2003);

- Desenvolvimento de sensores de pressão e de detecção de gases (SUN,1998) (KLEPS,2001) (GHODSIAN,1998) (SALANC,2003) (BADI,2004).

Nas aplicações de FED's em dispositivos integrados, microestruturas de Si, devido à sua compatibilidade com a tecnologia microeletrônica, têm desempenhado um papel importante em diversas áreas, como por exemplo: na microscopia de força atômica (AFM - “atomic force microscopy") (RESNIK,2003) (JÄNCHEN,2002), em microeletrodos para análise química (THIEBAUD,2000) e em medidas de biopotencial (GRISS, 2001) ou estimulação celular (HEUSCHKEL,2002).

Desta forma, várias pesquisas estão direcionadas para a obtenção de micropontas de Si visando a aplicação em emissores dos dispositivos FE, e sugerem diversas técnicas para a sua fabricação (FURSEY,2003) (MADOU,1997) (FUNG,2001) (GALEAZZO,2001) (DANTAS,2006B). Nessas pesquisas, busca-se a possibilidade de integração dos dispositivos (catodo e anodo em um mesmo substrato, por exemplo) além da eficiência, 
durabilidade, estabilidade das suas características elétricas. Aliado a essa busca na melhoria da obtenção e do desempenho das micropontas, surge também a necessidade de caracterizar eletricamente os referidos dispositivos, por meio de uma instrumentação dedicada, que possibilite polarizar os eletrodos em diferentes faixas de tensão, da ordem de centenas a alguns milhares de volts, e que viabilize a extração de correntes elétricas que variem em várias ordens de grandeza (principalmente entre nanoampères a microampères). Além disso, esta instrumentação deve apresentar um grau de liberdade para a polarização dos dispositivos de diferentes formas: tensão contínua ou rampa variável de tensão, e com intervalos de polarização e de aquisição diferenciados. Ademais, por meio da visualização gráfica dos resultados obtidos durante os experimentos, possibilitar alterar os parâmetros de polarização e, ao final dos ensaios elétricos, permitir o armazenamento dos dados coletados.

No GSIM (Grupo de Sensores Integráveis e Microssistemas) da EPUSP (Escola Politécnica da Universidade de São Paulo) está sendo desenvolvido um trabalho relacionado à fabricação de micropontas de Si a partir da técnica HI-PS (Hidrogen Implantation - Porous Silicon) (DANTAS,2006B) para serem aplicadas em sensores que operem através do princípio de emissão de campo. Por esta razão surgiu a necessidade de utilizar-se um sistema dedicado, que fosse capaz de polarizar os dispositivos em uma ampla faixa de tensão e extrair suas características elétricas.

Partindo-se de tal necessidade, propôs-se o desenvolvimento de uma instrumentação dedicada, composta por hardware e software, e projetada sob o conceito de instrumentação virtual, com o objetivo de atender as seguintes características:

- Faixa de tensão para polarização entre 0 a 4 kV;

- Opção de escolha de escalas de corrente diferenciadas (compromisso entre nível de corrente e resolução);

- Seleção de escala de corrente automática ou manual;

- Possibilidade de alterar os parâmetros dos ensaios "on-line", sem a necessidade de interromper o experimento;

- Apresentar, durante os ensaios, gráficos simultâneos ou não na tela do computador, como por exemplo, I-t; V-t; V-I;

- Armazenamento dos dados para posterior análise;

- Escolha de três modos de operação: Controle Manual, Controle de Tensão constante e Controle de Corrente constante;

- Facilidade de operação pelos usuários, e;

- Baixo custo. 
Nesse contexto, foram estabelecidos os seguintes objetivos específicos para este trabalho:

- Desenvolver o hardware da fonte de alta tensão e seu sistema microcontrolado de gerenciamento;

- Implementar um software de IHM (Interface Homem-Máquina) através de um computador usando linguagem de alto nível direcionada a objetos com capacidade de se comunicar com o hardware através da porta Serial RS-232.

- Apresentar graficamente cinco tipos de gráficos "on-line": I-t (Corrente versus tempo), V-t (Tensão versus tempo), V-I (Tensão versus Corrente), I-t e V-t simultâneos e o gráfico de Fowler-Nordheim. E armazenar os dados adquiridos durante os ensaios dos dispositivos emissores de campo.

\subsection{ORGANIZAÇÃO DO TRABALHO}

No capítulo dois são apresentados aspectos gerais relacionados aos FED's e à instrumentação virtual.

A descrição dos materiais utilizados, metodologia aplicada no desenvolvimento deste trabalho e as instruções de operação do equipamento serão apresentadas no terceiro capítulo.

No quarto capítulo são apresentados e discutidos alguns resultados alcançados, e comparações de desempenho entre o sistema desenvolvido e equipamentos comerciais encontrados no mercado.

A seguir, no quinto e sexto capítulos, são destacada a conclusão e as projeções futuras deste projeto, respectivamente. 


\section{REVISÃO BIBLIOGRÁFICA}

\subsection{DISPOSITIVOS EMISSORES DE CAMPO}

FED's são dispositivos cujo funcionamento baseia-se no fenômeno mecânico-quântico de emissão de elétrons que passam, através da barreira de potencial (por efeito de tunelamento), do material (sólido ou líquido) para o vácuo devido à aplicação de elevados campos elétricos próximo à superfície do material (XU,2005) (Fig. 2.1).

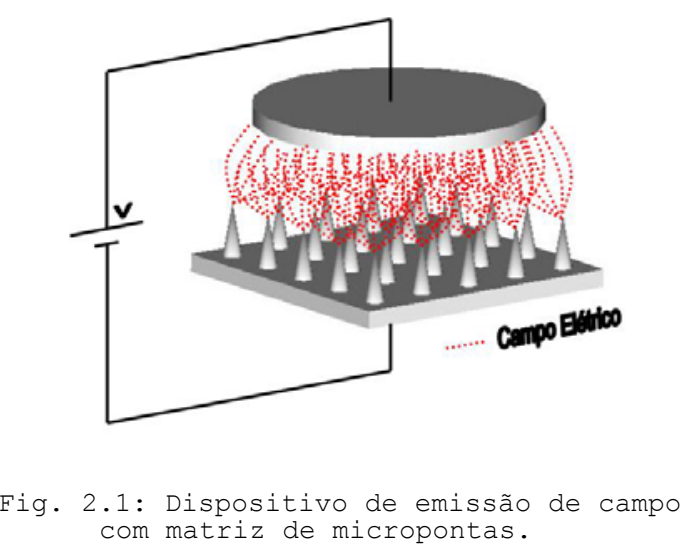

A densidade de corrente de emissão de elétrons a partir de uma microponta obedece a relação proposta por Fowler-Nordheim (FURSEY,2003), (FURSEY,2005), descrita a seguir:

$$
J=\frac{c_{1 .} E^{2}}{t^{2}(y) \cdot \phi} \cdot \exp \left(-c_{2} \cdot \frac{\phi^{\frac{3}{2}}}{E} \vartheta(y)\right)
$$

onde:

$J$ é a densidade de corrente de emissão $\left(\mathrm{em} \mathrm{A} / \mathrm{cm}^{2}\right)$,

$\phi$ é a função trabalho do material da microponta (em eV).

$c_{1}$ e $c_{2}$ são termos expressos por relações de constantes universais dados por $\mathrm{e}^{3} / 8 \pi h e$ $8 \pi 2^{1 / 2} \mathrm{~m} / 3 \mathrm{e} h$, respectivamente. Seus valores são: $\mathrm{c}_{1}=1,541 \times 10^{-6} \mathrm{AeV}\left(\mathrm{V}^{-2}\right)$ e $\mathrm{c}_{2}=$ $6,831 \times 10^{-9} \mathrm{eV}^{-3 / 2} \mathrm{Vm}^{-1}$.

$t(y)$ é um fator pré-exponencial cujo valor é próximo de 1 (FURSEY,2005) e $\vartheta(y)$

(função de Nordheim) é uma função elíptica de $y$ (DOLAN,1953), onde $y=3,79 \cdot 10^{-4} \cdot \sqrt{E} / \phi$. 
Os valores de $\vartheta(y)$ e $y$ são introduzidos na equação de F-N para considerar o efeito da carga-imagem (WISITSORAT,2002). Estes valores são tabelados e podem ser encontrados na literatura (BURGESS,1953).

$E$ é o campo elétrico entre os dois eletrodos planos (neste caso considerado semelhante a um capacitor de placas paralelas), sendo descrito por $E=V / d$, onde $V$ é o potencial elétrico aplicado (em volts) e $d$ é a distância de separação entre os eletrodos (em cm) (WISITSORAT,2002).

Todavia, em superfícies com irregularidades (com a presença de micropontas, por exemplo), o campo elétrico é variado, possuindo maior intensidade na extremidade ou muito próximo à superfície do emissor (microponta) (WISITSORAT,2002). Neste caso, para validar a equação de Fowler-Nordheim, é considerado um fator adimensional de aumento do campo elétrico em uma superfície com micropontas em relação a uma superfície plana, conhecido como fator de enriquecimento $(\gamma)$ (DANTAS,2006B).

Um dispositivo FE pode ser fabricado com os seus eletrodos integrados ou não. A Figura 2.2(a), ilustra um exemplo de dispositivo integrado, constituído por uma matriz de microagulhas ou micropontas (elementos denominados emissores ou catodos), cada uma localizada em uma cavidade circundada por um eletrodo de "porta" (elemento denominado "gate electrode" ou anodo) (TALIN,2001) (DEBSKI,2001) (JENKINS,1993).

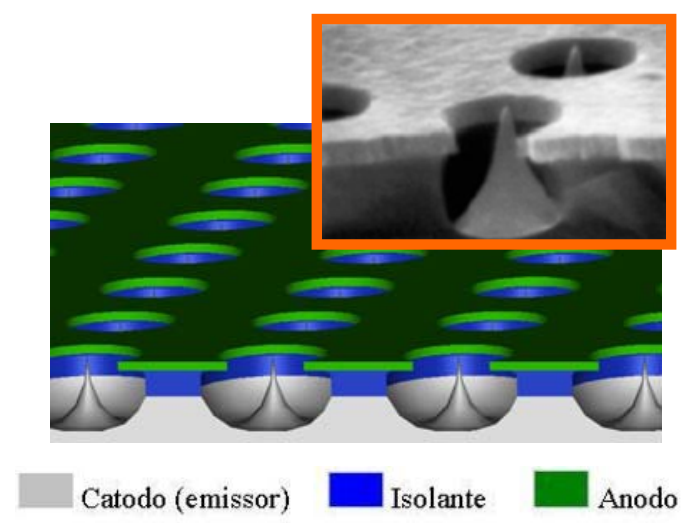

(a)

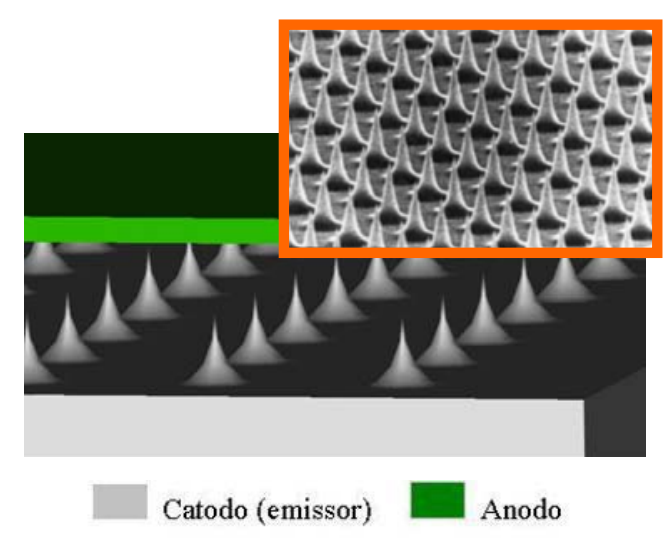

(b)

\footnotetext{
Fig. 2.2: Sistema Catodo/Anodo integrado (a) e Sistema não integrado (b).
}

Exemplos de FED's não integrados são apresentados na Fig. 2.2(b). Neste exemplo, o eletrodo externo (anodo) deve ser posicionado o mais próximo possível ao emissor para que o fenômeno de emissão ocorra com menor potencial de polarização (HUQ,2000).

Para produzir elevados campos elétricos com potenciais elétricos relativamente baixos, os FED's são fabricados com materiais específicos (preferencialmente com baixa função 
trabalho) e têm a geometria do elemento emissor otimizada, geralmente apresentando-se na forma de microagulhas, cujo raio de curvatura varia de dezenas de angstrons a alguns micrometros. Desta forma, os processos utilizados na fabricação dos dispositivos FE integrados devem proporcionar tanto a obtenção de micropontas uniformes com diâmetros extremamente reduzidos, como pequenas distâncias entre o emissor e o eletrodo de porta (DEBSKI,2001) (no caso de um sistema catodo/anodo integrado).

Apesar de diversos materiais (entre eles destacam-se o molibdênio, a platina, etc, e recentemente nanotubos de carbono) e técnicas (usualmente processos em soluções químicas e por plasma) serem utilizados na fabricação de dispositivos FE, muitos dos processos sugeridos tornam-se inviáveis de serem implementados industrialmente, devido a problemas como complexidade, alto custo e principalmente a baixa compatibilidade com as tecnologias de Microeletrônica, o que dificulta o emprego de tais dispositivos em circuitos integrados. Desta forma, uma maneira de promover tal compatibilidade consiste em explorar-se a fabricação das "micropontas" em $\mathrm{Si}$, pois por meio de processos de microusinagem, é possível fabricar micropontas ou microagulhas com elevada razão de aspecto compatíveis com outros circuitos de condicionamento do sinal que facilitam sua integração.

\subsection{INSTRUMENTAÇÃO VIRTUAL}

Com o desenvolvimento da tecnologia, em especial no que se diz respeito às áreas relacionadas às engenharias elétrica e eletrônica, tornou-se altamente necessário e de fundamental importância mensurar e controlar grandezas elétricas em incontáveis aplicações nos mais diversos segmentos, dentro e fora da própria engenharia (GOLDBERG, 2000), assim como caracterizar eletricamente dispositivos e circuitos eletrônicos disponíveis comercialmente ou em desenvolvimento, no âmbito ou não da pesquisa científica. Seja para a pesquisa básica e aplicada ou para projetos de desenvolvimento, exige-se cada vez mais que a instrumentação seja modernizada (disponibilizando, por exemplo, maior capacidade de aquisição de dados e de processamento, armazenamento de dados, etc), e que disponibilize várias ferramentas para auxiliar na caracterização e extração dos parâmetros elétricos. Neste caso, para o âmbito da pesquisa, devem-se acrescentar os fatores tempo de desenvolvimento e custo da implementação da instrumentação dedicada.

A partir do desenvolvimento de redes de computadores tornou-se possível a constituição de sistemas de medição computador-instrumentação, separadamente. 
Usando-se, portanto qualquer um dos diversos formatos de comunicação: RS-232 à GPIB, USB, Ethernet, SCSI. Mais recentemente, com a introdução de alguns novos protocolos de comunicação de rádio freqüência, como o "Bluetooth", Wi-Fi ou ZigBee, o computador não deve necessariamente estar conectado fisicamente com o instrumento. Atualmente, usando protocolo TCP/IP de Internet de alta velocidade, é possível fazer com que a informação seja transportada pela rede, e então processada, mostrada, controlada e ou distribuída em qualquer lugar no mundo (GOLDBERG, 2000).

Associado às tendências da instrumentação atual e aliado à necessidade do desenvolvimento de um sistema dedicado para caracterização elétrica em experimentos efetuados com dispositivos de emissão de campo, é que foi concebido o presente trabalho, no qual o principal enfoque do estudo está no desenvolvimento de um sistema composto por uma fonte de alta tensão constituída de módulos de geração de tensões primárias e processamento de informações, aliada a um ambiente visual, cujo programa instalado em um microcomputador padrão IBM-PC possibilite toda a manipulação da fonte e registro dos dados de ensaio.

Em princípio a instrumentação virtual, cujo conceito está representado na Figura 2.3 (BORGES,2002), é uma proposta para controle de processos, envolvendo equipamentos de medida, software e computador, tornando possível a realização de controle do fluxo de informações entre dispositivos através de interfaces padronizadas para comunicação entre homem-máquina. O nome "instrumento virtual" deriva dos aspectos realistas de operação que dizem respeito aos instrumentos clássicos (CRISTALDI, 1999) como, por exemplo, voltímetros, osciloscópios entre outros, e foi originalmente concebido como uma ferramenta de desenvolvimento que automatiza procedimentos laboratoriais e industriais (MARINO,2000).

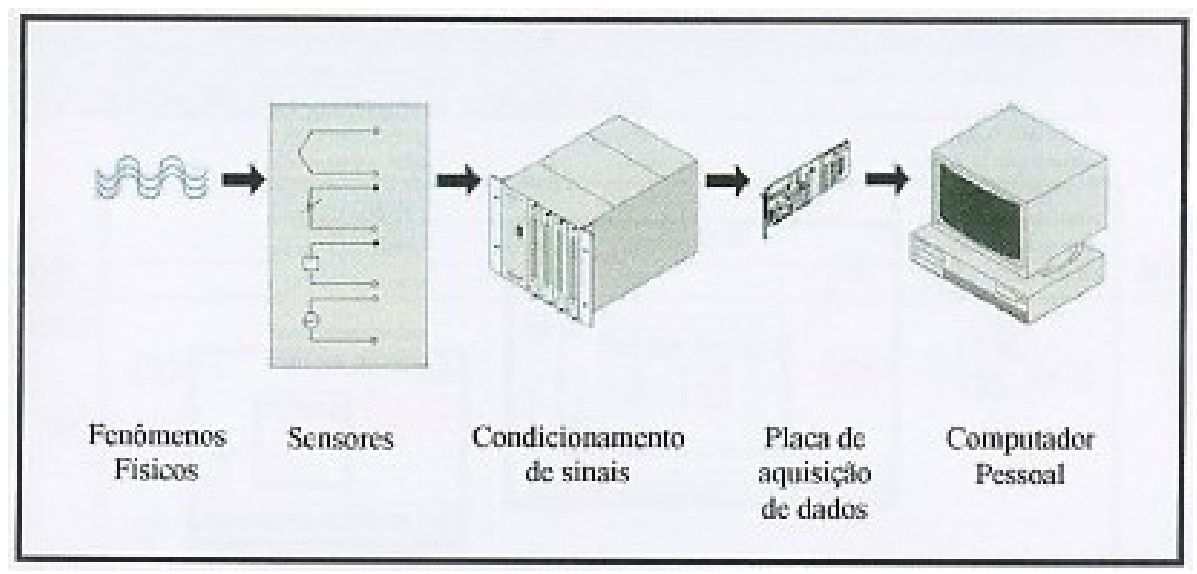

Fig. 2.3: Exemplo genérico de ferramenta de instrumentação virtual (Placas de aquisição, computadores, software), representando a instrumentação virtual. 
O uso da instrumentação virtual tem se destacado também no contexto de controle e monitoração de processos no âmbito industrial, na pesquisa e no desenvolvimento de eletrônica embarcada (BECK, 2001)

A instrumentação virtual é aplicada em diversas áreas, como:

- Gerador de Sinal Digital Programável (AGUIRRE,2006);

- Análise de Sistemas eletrônicos de Potência (SPANIK,2006);

- Monitoramento de máquinas de Indução (EREN,1998);

- Monitoramento de condições de Rolamentos Integrados (WANG,2000);

- Avaliação clínica de funções cardiovasculares e autonômicas (BENITEZ,2000);

- Medições de altas correntes pulsadas de fontes de alta potência de lasers de alta tensão (LI,2005);

- Medição de emissão de correntes harmônicas (GEORGE,2003);

\subsubsection{PARÂMETROS QUE DESCREVEM A INSTRUMENTAÇÃO}

O sistema de instrumentação virtual pode ser descrito por quatro parâmetros: modelo de programação, estrutura de controle, latência e dispositivos específicos (SPOELDER,1999). O primeiro parâmetro está relacionado ao modelo de programação - a programação é gráfica, ou seja, não é necessário digitar linhas de códigos para a execução de determinada atividade. O segundo parâmetro que descreve a instrumentação virtual, é a estrutura de controle - diagramas de blocos que executam seqüências lógicas são dispostos de forma a executar o controle de um processo. A latência, citada como terceiro parâmetro, está relacionada ao tempo de resposta entre a mensagem do software e os instrumentos, ou seja, o tempo em que um instrumento leva para responder a um comando vindo do software. E para completar, os dispositivos específicos - como, por exemplo, osciloscópio e fonte de tensão formam o conjunto descrito como o quarto parâmetro de um sistema instrumentação virtual.

\subsubsection{HARDWARE E SOFTWARE}

Os microprocessadores e técnicas de software têm tornado a instrumentação virtual uma forma sofisticada de controle (BORGES,2002), e muitos padrões de comunicação entre hardware têm sido estabelecidos (WANG,2000), (TANER,1997) para oferecer suporte aos dispositivos e interface para instrumentação.

A eficiência do projeto da instrumentação virtual está relacionada ao próprio processo de programação, onde o usuário não precisa ser especialista para 
implementar o controle de um instrumento, para que alguma atividade seja executada (BORGES,2002). As interfaces assemelham-se aos displays dos instrumentos reais, ou seja, para que os indivíduos acostumados a trabalhar com instrumentação convencional também possam se adaptar a um novo tipo de ambiente (CRISTALDI,1999) onde é possível realizar controles e medições de diversos parâmetros simultaneamente, o que propicia a transportabilidade da instrumentação convencional.

O software que representa a utilização da instrumentação virtual e que se mostra como importante ferramenta para o desenvolvimento desse projeto é o programa nomeado de "HighVolt Controller". O HighVolt Controller é um programa que possibilita a integração entre hardware e software e foi desenvolvido no ambiente de programação visual Delphi versão 7.0 produzido pela empresa Borland.

De acordo com Wang (WANG,2000), as interfaces elaboradas para controle via instrumentação virtual, são responsáveis por:

- Manter contato com a unidade central de processamento e executar diferentes testes e funções para medição de sinais;

- Executar o controle de fluxo de dispositivos periféricos (como sensor e circuitos de processamento de sinais) através de interfaces padrões;

- Adquirir mais de um tipo de dado e mostrar o resultado desta aquisição;

- Acompanhar o fluxo de informação do controle de dispositivos periféricos (como sensor e circuitos de processamento de sinal) de forma a permitir futuras análises;

- Possibilitar a exibição e análise de dados adquiridos.

\subsubsection{DESENVOLVIMENTO DE UMA INSTRUMENTAÇÃO DEDICADA PARA CARACTERIZAÇÃO DE DISPOSITIVOS EMISSORES DE CAMPO}

Associado às tendências da instrumentação atual aliado a necessidade de se desenvolver um sistema dedicado para caracterização elétrica de dispositivos emissores de campo elétrico, foi concebido o presente trabalho, onde o principal enfoque de estudo está no desenvolvimento de um sistema formado por uma fonte de alta tensão composta por módulos de geração de tensões primárias e de processamento dos dados, interligada a um ambiente gráfico aplicando-se o conceito de instrumentação virtual, cujo programa computacional, executado na plataforma PC(x86) com sistema operacional Windows XP, possibilite o controle da fonte de alta 
tensão e o armazenamento dos dados do ensaio, conseqüentemente facilitando o estudo dos dispositivos FE.

\subsection{EMISSÃO DE ELÉTRONS}

Existem diversos tipos de fenômenos de emissão de elétrons, dentre os quais destacam-se a emissão termoiônica e a emissão de campo, que é o foco no que diz respeito a dispositivos FE (DANTAS,2006B).

\subsubsection{EMISSÃO TERMOIÔNICA}

A emissão termoiônica ocorre quando um material, geralmente um metal, é aquecido com a passagem de uma corrente elétrica a elevadas temperaturas ( $>$ $1000{ }^{\circ} \mathrm{C}$ ), fornecendo aos elétrons energia suficiente para "vencerem" a funçãotrabalho do material e serem emitidos do mesmo (MAMMANA,2000), (JENSEN,2001B).

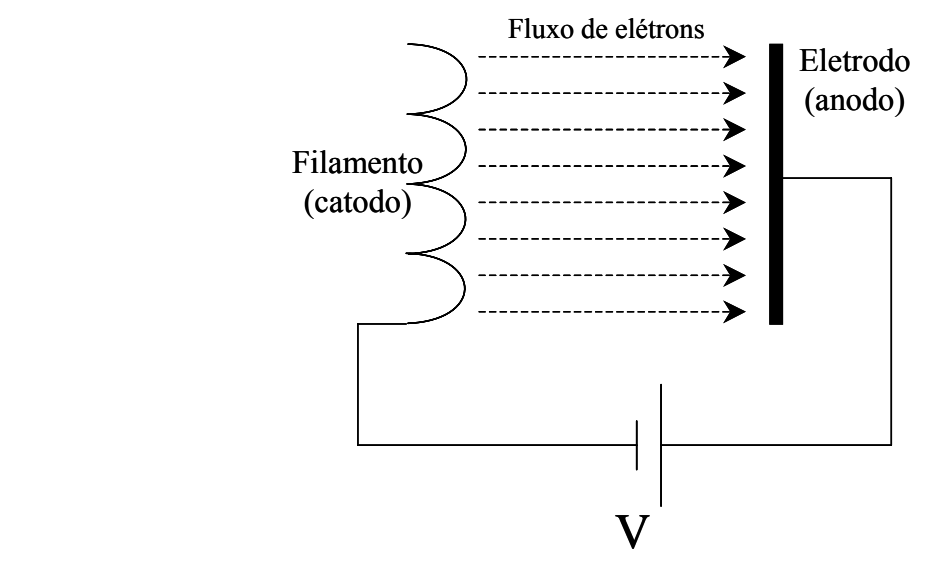

Fig. 2.4: Esquema ilustrativo de um dispositivo de emissão termoiônica.

Para "capturar", direcionar ou acelerar os elétrons emitidos do material aquecido, em geral um filamento (catodo quente), um eletrodo (anodo) é mantido em um potencial elevado em relação ao catodo (da ordem de dezenas de quilovolts), como ilustra a Figura 2.4.

A corrente elétrica que pode ser emitida por unidade de superfície do catodo depende fundamentalmente da temperatura de operação e da função de trabalho do material empregado na sua construção. Ao atingir o valor mínimo de energia térmica aplicada ao catodo (valor determinado pela função de trabalho do material), a emissão termoiônica se inicia e aumenta rapidamente com a elevação da temperatura.

A emissão termoiônica, apesar de ser uma tecnologia consolidada e do tempo de vida relativamente elevado dos dispositivos, apresenta características indesejáveis, 
como por exemplo: elevada temperatura de operação $\left(>1000{ }^{\circ} \mathrm{C}\right)(\mathrm{ZHU}, 2001)$, alto consumo de potência devido à baixa eficiência deste tipo de emissão (SUN,1998), necessidade de um período prévio de aquecimento do catodo para iniciar o processo de emissão (SUN,1998), robustez e fragilidade dos dispositivos, limitando-os a determinados tipos de aplicações (XU,2005), (ZHU,2001).

\subsubsection{EMISSÃO DE CAMPO}

O princípio do efeito de emissão de campo baseia-se na aplicação de um campo elétrico elevado para extrair elétrons do material (FURSEY,2003), (XU,2005). A eficiência desta emissão é milhões de vezes superior a outros processos conhecidos de emissão de elétrons (FURSEY,2003).

Este fenômeno foi descoberto por R.W. Wood em 1897, porém o autor não conseguiu uma explicação teórica plausível para justificá-lo devido a limitações tecnológicas da época. Posteriormente vários pesquisadores estudaram o fenômeno de emissão de campo, destacando-se entre eles Schottky, Millikan, Eyring, Gossling e Lauritsen (MAMMANA,2000), (FURSEY,2005). Schottky previu teoricamente (em 1923) que a barreira de potencial na superfície do material era inferior ao campo elétrico aplicado para emissão dos elétrons, fato posteriormente comprovado experimentalmente (FURSEY,2005). Em 1926 Millikan, Eyring e Gossling descobriram que a corrente de emissão não era afetada por temperaturas até $1500^{\circ} \mathrm{K} \mathrm{e}$, posteriormente, Millikan e Lauritsen descreveram que a mesma variava exponencialmente com o potencial elétrico aplicado (FURSEY,2005).

Mas foi em 1928 que Fowler e Nordheim explicaram com êxito a emissão de campo como sendo um fenômeno mecânico-quântico de elétrons que passam (por efeito de tunelamento) através da barreira de potencial do material (sólido ou líquido) para o vácuo (XU,2005), (FURSEY,2005). Esta teoria descreve a dependência da corrente de emissão de elétrons em relação ao campo elétrico aplicado e a função trabalho do material (FURSEY,2005).

Para que este fenômeno ocorra, é necessário que o campo elétrico próximo à barreira de potencial na interface metal-vácuo (emissor-vácuo) seja elevado o suficiente (da ordem de $10^{7}-10^{9} \mathrm{~V} / \mathrm{cm}$ ) afim de que os elétrons possuam uma significativa probabilidade de tunelamento do sólido para o vácuo (FURSEY,2003), (MAMMANA,2000), (SUN,1998). A Figura $2.5 \quad$ (FURSEY,2003), 
(MAMMANA,2000), (SUN,1998) ilustra um modelo para a barreira de potencial com e sem aplicação de um campo elétrico externo.

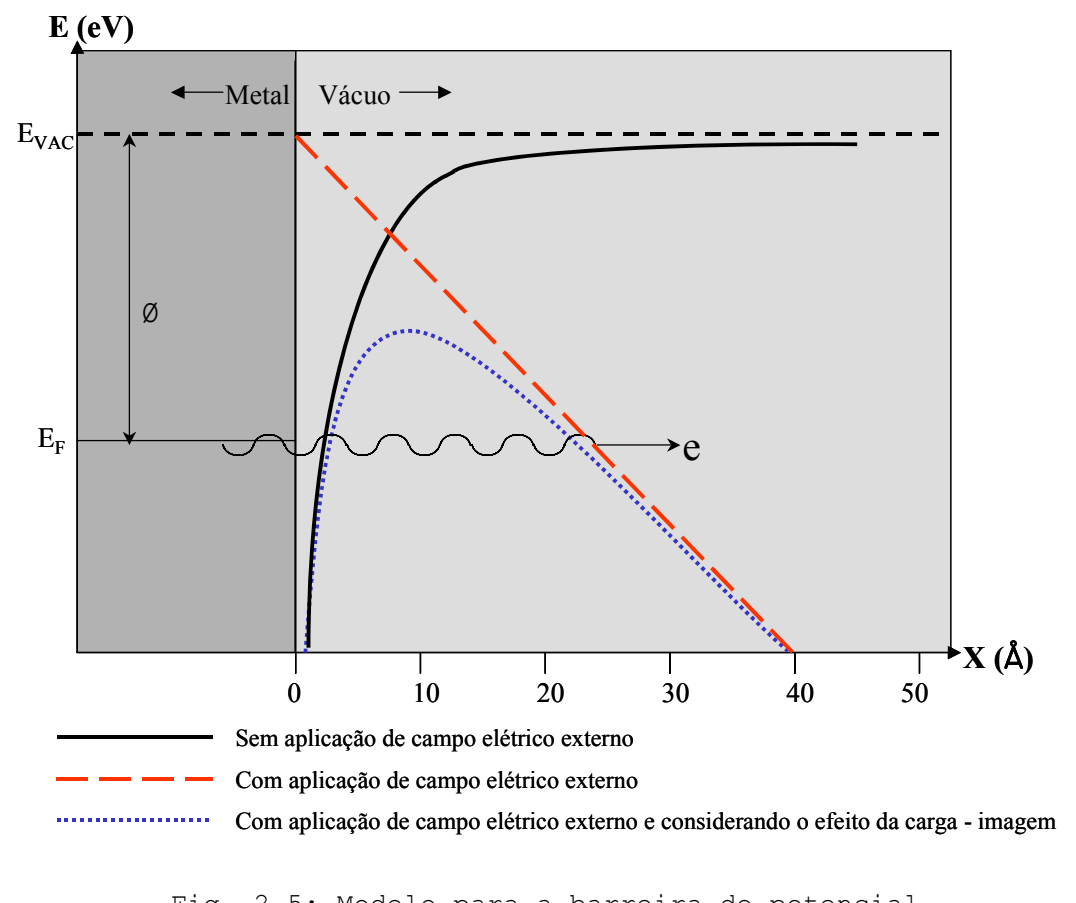

em função da distância em relação à superfície do metal (FURSEY, 2003), (MAMMANA, 2000),
(SUN, 1998), onde $E=$ energia potencial, X = distância da superfície do material, Evac =
nível de vácuo, $E_{F}=$ nível de Fermi, $\phi=$ função trabalho do metal.

A curva pontilhada da Figura 2.5 representa a modificação da barreira de potencial considerando-se o efeito da carga-imagem devido à interação entre os elétrons no vácuo e o catodo de metal, sendo descrita por $V=\phi-E x-\frac{q^{2}}{16 \pi x}$, para $x>x_{0}$ (WISITSORAT,2002).

\section{MATERIAIS E MÉTODOS}

Este capítulo tem por objetivo apresentar os aspectos relevantes do projeto, os materiais e a descrição da metodologia utilizada para a implementação do Sistema de Caracterização Elétrica de Dispositivos Emissores de Campo (SCEDEC).

O SCEDEC consiste em um sistema de baixo custo, com alta confiabilidade e precisão, que apresenta integração entre software e hardware num contexto de instrumentação virtual, direcionado para aplicações em processos de caracterização elétrica de dispositivos de emissão de campo.

A Figura 3.1 mostra um diagrama de blocos das partes fundamentais do sistema desenvolvido: 


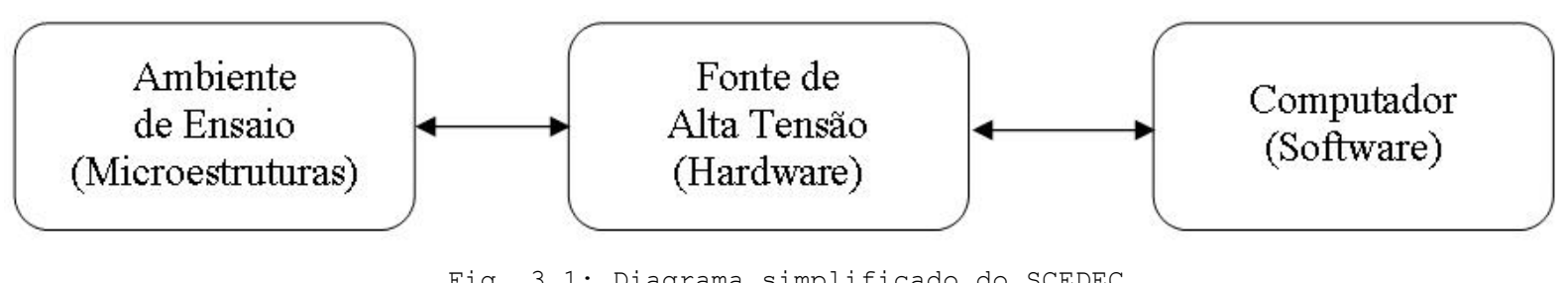

\subsection{OS MATERIAIS}

Os materiais utilizados no desenvolvimento desse trabalho estão relacionados ao hardware, ao software e aos mecanismos que possibilitam a integração hardwaresoftware através da comunicação serial. A seguir, são detalhados os principais materiais utilizados: 
3.1.1 COMPONENTES ELETRÔNICOS

\begin{tabular}{|c|c|c|c|c|}
\hline DESCRIÇÃO & MODELO & FABRICANTE & PRINCIPAIS CARACTERÍSTICAS & FUNÇÃO \\
\hline$\mu \mathrm{C}$ & $\mathrm{PIC} 18 \mathrm{~F} 485 \mathrm{I} / \mathrm{P}$ & MICROCHIP & $\begin{array}{l}\text { Três interrupções externas; } \\
\text { o Um timer/contador de } 8 \text { bits; } \\
\text { o Dois timers de } 16 \text { bits; } \\
\text { o Portas de comunicação I²C, SPI, } \\
\text { USART (RS-232/RS-485), Paralela; } \\
\text { o Velocidade de processamento acima } \\
\text { de 10MIPS, entre outras. }\end{array}$ & $\begin{array}{l}\text { O microcontrolador é responsável pelo } \\
\text { gerenciamento de todo o hardware. É um } \\
\text { componente que possui uma memória, onde é } \\
\text { gravado um arquivo hexadecimal. Esse } \\
\text { arquivo é oriundo de um compilador que } \\
\text { converte o programa feito em "linguagem C", } \\
\text { programa esse que determina quais funções } \\
\text { que serão executadas pelo componente. A } \\
\text { página inicial da especificação técnica desse } \\
\text { microcontrolador, fornecida pelo fabricante, } \\
\text { encontra-se no anexo 8.1. }\end{array}$ \\
\hline $\begin{array}{l}\text { AMPLIFICADOR } \\
\text { OPERACIONAL }\end{array}$ & LF353 & FARCHILD & $\begin{array}{l}\text { ○ Ganho em ampla largura de banda - } \\
4 \mathrm{MHz} \text {; } \\
\circ \text { Alta taxa de rampa de tensão - } \\
13 \mathrm{~V} / \mu \mathrm{s} \text {; } \\
\circ \text { Alta impedância de entrada }-10^{12} \Omega \text {. }\end{array}$ & $\begin{array}{l}\text { Esse amplificador operacional foi usado } \\
\text { no circuito responsável pela geração do sinal } \\
\text { senoidal de } 49 \mathrm{kHz} \text {, pois esse componente } \\
\text { apresenta um bom funcionamento nessa faixa } \\
\text { de freqüência. A página inicial da } \\
\text { especificação técnica desse amplificador } \\
\text { operacional, fornecidas pelo fabricante, } \\
\text { encontra-se no anexo 8.2. }\end{array}$ \\
\hline
\end{tabular}




\begin{tabular}{|c|c|c|c|c|}
\hline DESCRIÇÃO & MODELO & FABRICANTE & CARACTERÍSTICAS & FUNÇÃO \\
\hline $\begin{array}{l}\text { DRIVER / } \\
\text { RECEIVER }\end{array}$ & MAX232 & MAXIM & $\begin{array}{l}\text { ○ Tensão de alimentação de } 5 \mathrm{~V} \text {; } \\
\text { ○ Múltiplos drivers/receivers; } \\
\text { ○ Saídas drivers/reveicers tri-estados. }\end{array}$ & $\begin{array}{l}\text { Esse componente foi usado para } \\
\text { converter os níveis de tensão entre a porta } \\
\text { RS-232 e o microcontrolador para que a } \\
\text { comunicação do hardware com o software do } \\
\text { SCEDEC fosse possível. A página inicial da } \\
\text { especificação técnica desse componente, } \\
\text { fornecidas pelo fabricante, encontra-se no } \\
\text { anexo 8.4. }\end{array}$ \\
\hline
\end{tabular}

Tabela 3.1 Componentes utilizados no trabalho 


\subsubsection{RS-232}

Foi utilizado como interface de comunicação entre o computador e a fonte, a porta de comunicação serial do computador sob o padrão RS-232. As maiores dificuldades encontradas pelos usuários na utilização da interface RS-232 incluem pelo menos um dos seguintes fatores:

$\checkmark$ A ausência ou conexão errada de sinais de controle resulta em estouro do buffer ("overflow") ou travamento da comunicação.

$\checkmark$ Função incorreta de comunicação para o cabo em uso resulta em inversão das linhas de Transmissão e Recepção, bem como a inversão de uma ou mais linhas de controle ("handshaking”).

Felizmente, os drivers utilizados são bastante tolerantes aos abusos cometidos, e os circuitos integrados normalmente não são danificados.

\subsubsection{SOFTWARE - MPLAB-IDE}

Para o desenvolvimento do programa do microcontrolador foi aplicado o programa MPLAB-IDE que é desenvolvido pela empresa Microchip. O MPLAB-IDE é um programa gratuito disponibilizado no website do desenvolvedor (www.microchip.com), cujo ambiente de programação oferece todas as ferramentas necessárias para o desenvolvimento de programas de microcontroladores em um único ambiente. O MPLAB-IDE tem como base de programação a linguagem Assembler, porém para o desenvolvimento desse trabalho, foi utilizado um compilador que tem como base de programação a linguagem "C". Esse compilador foi desenvolvido pela empresa CCS e é compatível com o MPLAB-IDE. No MPLAB-IDE é possível, além de desenvolver os programas para microcontroladores, desenvolver aplicações e simulá-las, e quando possuir os emuladores, é possível também emular as aplicações em tempo real "on-line". 


\subsubsection{SOFTWARE - DELPHI}

Para o desenvolvimento do software que compõe o SCEDEC foi utilizado um programa para programação de linguagem em alto nível que é denominado Delphi versão 7.0 e foi instalado no sistema operacional Windows XP. O Programa Delphi utiliza como base de programação a linguagem "Pascal" e durante o desenvolvimento desse trabalho, utilizou-se recursos para desenvolvimento de menus, acionadores, displays, comunicação serial, banco de dados, visualizações gráficas, entre outras.

\subsubsection{CÂMARA PARA ENSAIOS ELÉTRICOS}

A Figura 3.2a ilustra a câmara de vácuo confeccionada no laboratório de Microeletrônica para ser utilizada nos ensaios elétricos dos dispositivos FE.

A função do posicionador, acoplado ao pino de suporte do catodo (Figura 3.2b), é alterar a distância entre as estruturas do anodo e catodo (Figura 3.2c) e avaliar a alteração da corrente de emissão em função de tal distância. A estrutura do anodo (Figura 3.2d), feita do mesmo material da câmara (aço inox), é parafusada ao pino de suporte, e possui extremidades arredondadas para desfavorecer descarga nesta região.

Como a alta tensão será aplicada no anodo, ele necessita ser isolado eletricamente das demais partes do sistema, o que foi realizado utilizando-se materiais cerâmicos (Figura 3.2e). Por fim, o substrato de Si contendo a matriz de micropontas dever ser fixado ao porta amostras através de isolantes de material plástico (Figura $3.2 f)$.

Com relação ao sistema de vácuo, a câmara não apresentou vazamentos que comprometessem seu funcionamento, possibilitando atingir pressões da ordem de $10^{-6}$ Torr. 


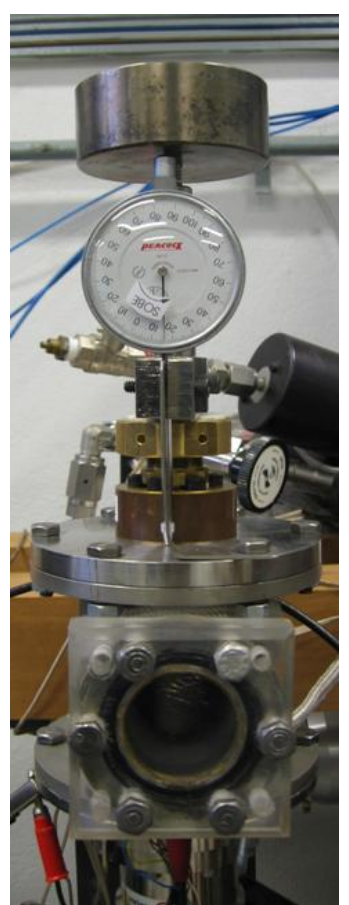

(a)

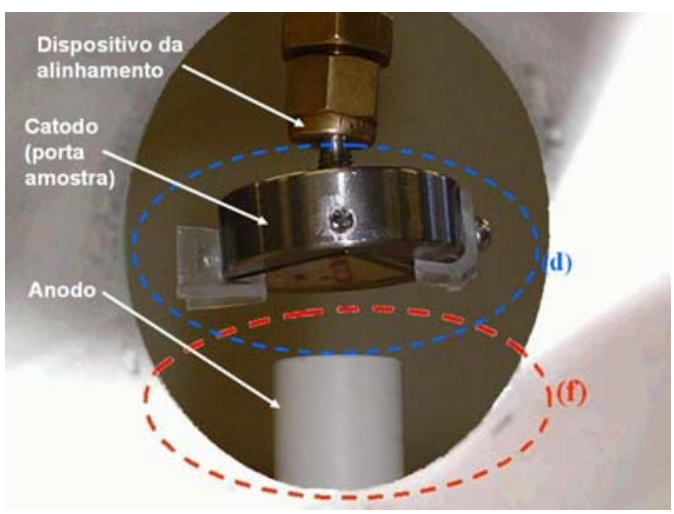

(c)

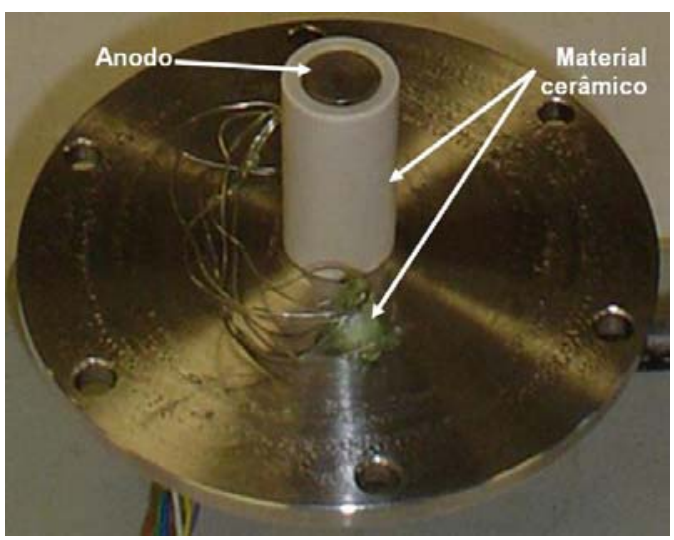

(e)

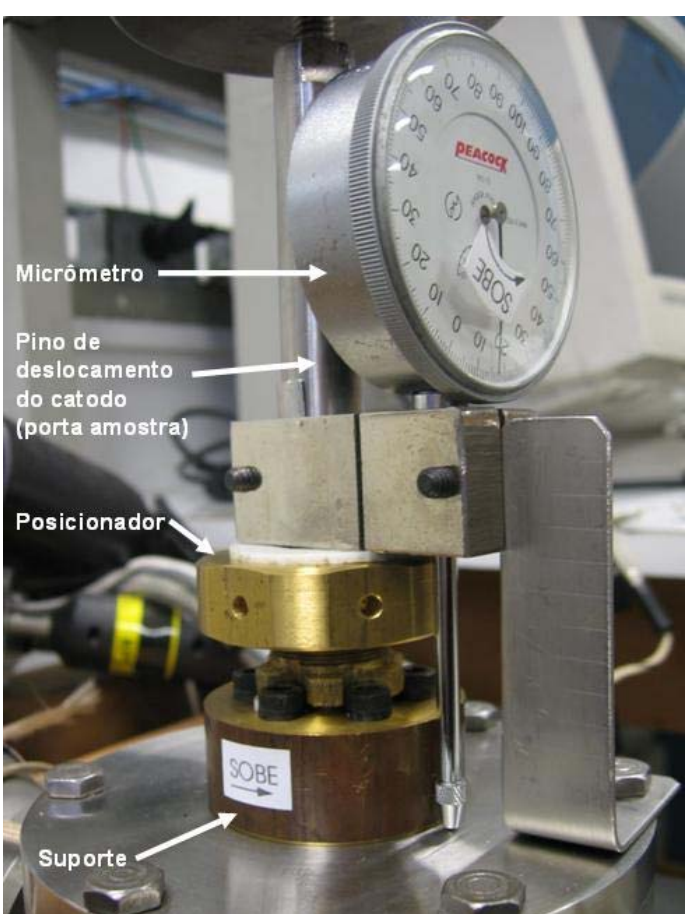

(b)

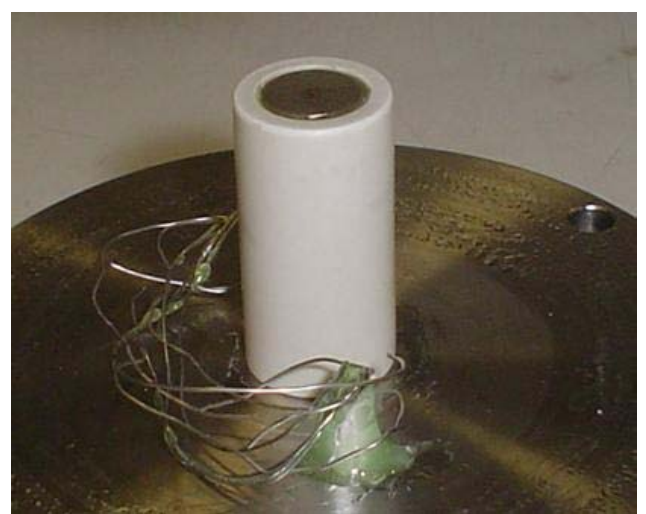

(d)

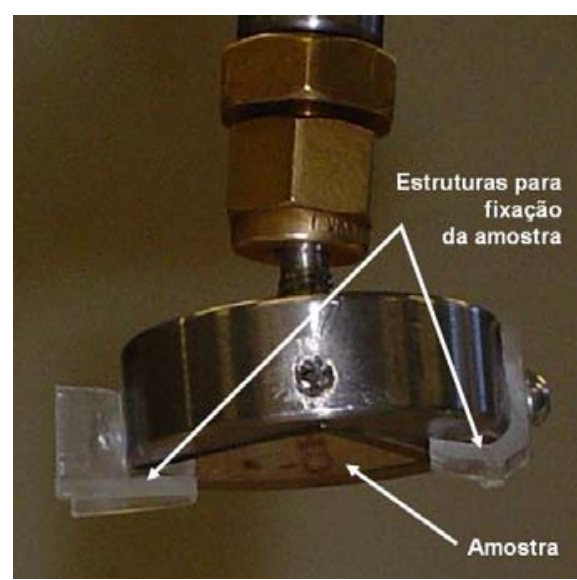

(f)

Fig. 3.2: (a) vista geral da câmara

(b) parte superior da câmara ilustrando o posicionador e micrômetro acoplados ao pino de suporte do catodo; (c) vista interna da câmara ilustrando o catodo e o anodo; (d) anodo; (e) flange inferior, que contém o anodo, isolado eletricamente do sistema por um material cerâmico; (f) detalhe do porta amostra. 


\subsection{ETAPAS PRÉ-ESTABELECIDAS PARA O PROJETO}

O desenvolvimento do trabalho foi escalonado em etapas (Fig. 3.3), sendo que a primeira etapa consiste no levantamento das principais características desejáveis para que o sistema pudesse atender as necessidades do processo de caracterização dos dispositivos emissores de campo em instrumentação virtual.

A segunda etapa foi desenvolvida através da elaboração do hardware da Fonte de Alta tensão, incluindo os circuitos das fontes de alimentação, programação do microcontrolador, estabelecimento do protocolo de comunicação além da estimulação do módulo de potência através de um circuito gerador de onda senoidal de $49 \mathrm{kHz}$.

Para a implantação da terceira etapa, que consiste em desenvolver um programa visual que possibilita o controle do experimento via computador, é utilizado o programa Delphi 7.0 para Windows, onde foi desenvolvido o instrumento virtual responsável pelo controle, manipulação e aquisição dos dados dos ensaios.

A quarta etapa envolve todo o processo de calibração e o processo de levantamento das características do sistema, bem como os acertos necessários para corrigir eventuais problemas detectados durante essa etapa.

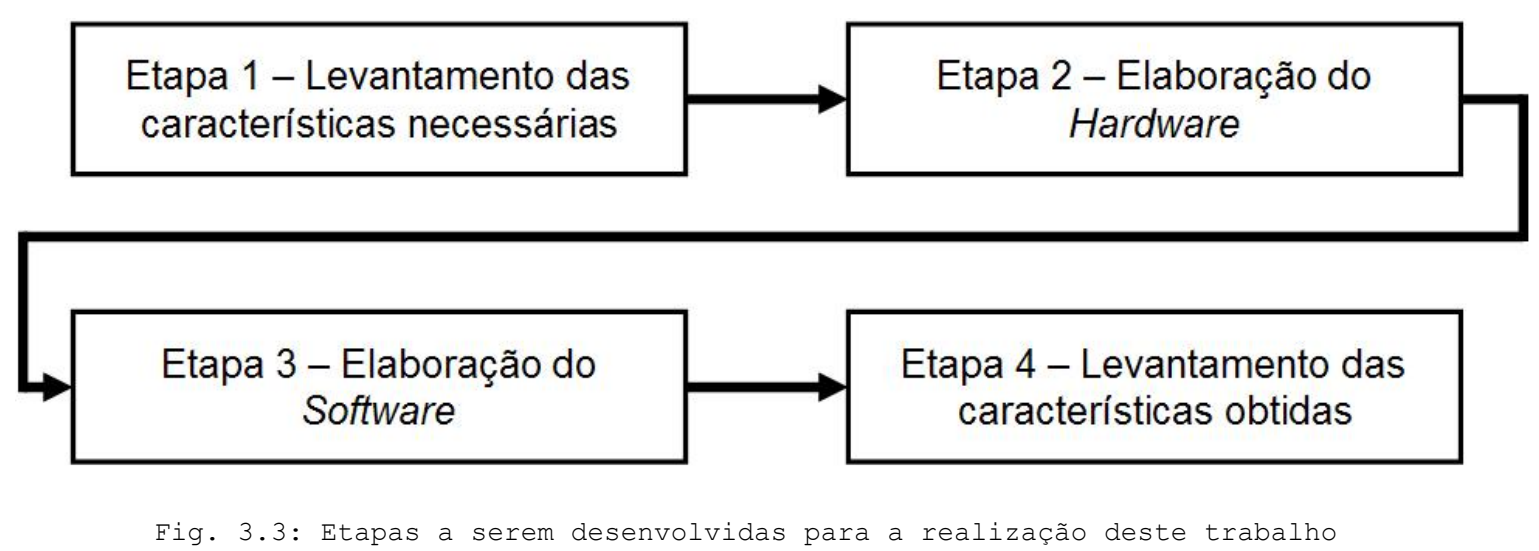

\subsection{METODOLOGIA APLICADA}

Uma das etapas fundamentais que permitiram a concepção do SCEDEC foi a etapa de desenvolvimento do hardware (Fonte de Alta Tesão) do Sistema. Durante essa etapa, foram elaborados levantamentos de especificações, referente à qual fonte deveria ser adotada como fonte de alta tensão. Foram consideradas várias alternativas entre as quais, a de aplicação de um Fly-back e aplicação de uma Bobina de centelhamento automotivo, porém a que proporcionou uma melhor qualidade de tensão de saída, foi o módulo de 
potência. O módulo de potência é uma fonte de alta tensão contínua alimentado com uma tensão de $110 \mathrm{Vac}$ e calibrado para sempre manter uma tensão constante de $1,5 \mathrm{kVdc}$ nos terminais de saída. Esse módulo foi retirado de um equipamento sem uso e foram feitas alterações relacionadas principalmente ao circuito de atuação para que ele pudesse atender as especificações do presente trabalho.

Após ser estipulada a fonte de alta tensão que seria utilizada no Sistema, foram levantas as características do módulo de potência e verificou-se que o melhor desempenho era a través da excitação do módulo, aplicando-se uma tensão senoidal com freqüência de $49 \mathrm{kHz}$ e que a variação da tensão de saída fosse implementada através da variação da amplitude dessa tensão.

Para a transferência de dados entre o computador (software) e o hardware ocorrer, há a necessidade de um protocolo de comunicação. Para tal, foram desenvolvidos dois protocolos de transferência. Um relacionado à transferência de dados PC-FONTE (mais detalhado no capítulo 4.1.3) e outro relacionado à transferência de dados FONTE-PC (mais detalhado no capítulo 4.2.3).

Neste projeto foi utilizada comunicação serial no padrão RS-232, para a transferência dos dados. A metodologia de detecção de falhas na comunicação, foi implementada através da aplicação do Checksum.

Para interação do usuário com o Sistema, foi implementado um instrumento virtual através do desenvolvimento de um software utilizando-se o programa Delphi 7.0. O software desenvolvido foi denominado de "HighVolt Controller" e nele é possível, entre outras coisas:

- Analisar a comunicação no processo de Debug;

- Selecionar três modos de operação da fonte: Controle Manual, Controle de Corrente e Controle de Tensão;

- Visualizar as leituras durante o funcionamento;

- Selecionar a velocidade de atualização do gráfico e dos displays indicadores.

O sistema proposto é mostrado na Figura 3.4, onde é possível identificar o hardware (Fonte de Alta Tensão) e o microcomputador além do ambiente de ensaio desenvolvido: 


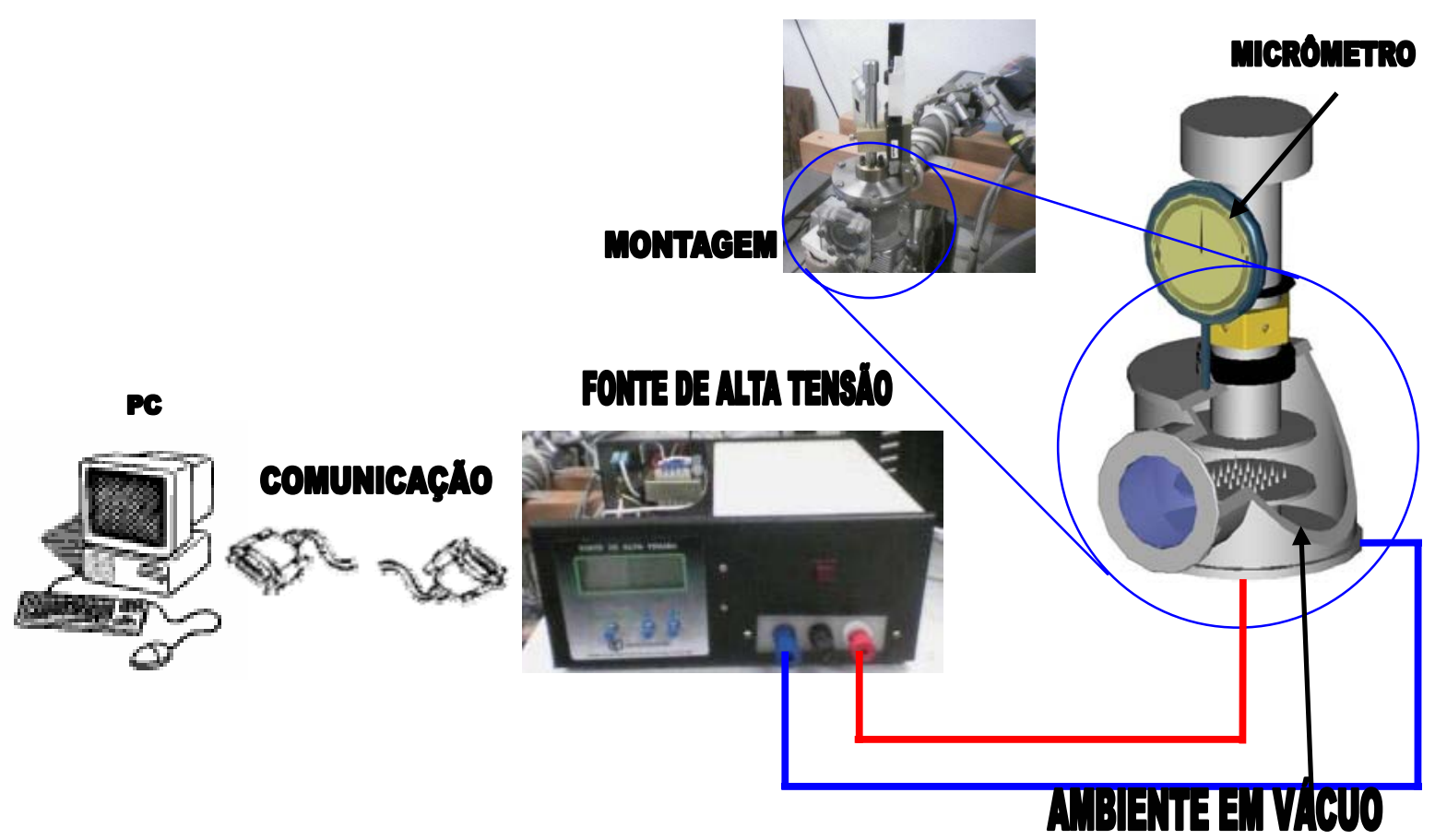

Fig. 3.4: Sistema de caracterização dos dispositivos FE

A Figura 3.5 mostra a metodologia de desenvolvimento utilizada para este projeto apresentando de uma forma geral, como as etapas descritas nesse capítulo foram desenvolvidas.

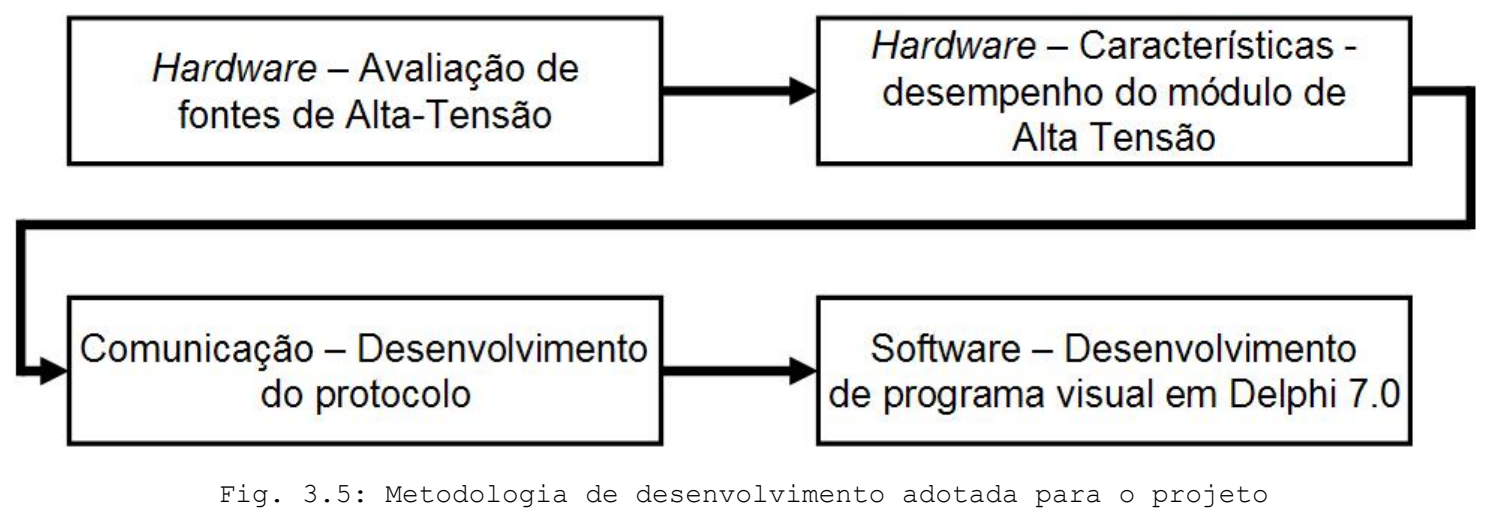

\subsection{CONSIDERAÇÕES FINAIS}

Este capítulo descreveu os principais materiais e a metodologia utilizada para o desenvolvimento deste trabalho, mostrando a importância e justificando as escolhas das tecnologias. Ao final encontra-se o diagrama de blocos ilustrando as etapas do projeto e uma breve descrição das atividades realizadas em cada etapa.

O próximo capítulo apresentará os resultados preliminares experimentais obtidos das atividades realizadas em cada etapa. 


\section{RESULTADOS EXPERIMENTAIS}

Adiante são discutidos o hardware e o software desenvolvidos nesse trabalho, onde o hardware é constituído pelo conjunto de componentes físicos do sistema (material eletrônico, placas, display etc.) e software, constituído por programas, rotina ou conjunto de instruções que controlam o funcionamento do SCEDEC, ou seja, são os programas desenvolvidos para o computador e para o microcontrolador da fonte de alta tensão.

\subsection{HARDWARE}

Na fonte de Alta Tensão, desenvolvida de acordo com o diagrama da Figura 4.1, foram utilizadas quatro fontes de tensão contínua, três referenciadas ao comum digital com valores de tensão de $+15 \mathrm{Vdc},-15 \mathrm{Vdc},+5 \mathrm{Vdc}$ e duas referenciadas ao Terra físico da rede elétrica com valor de tensão de $5 \mathrm{Vdc}$. Todo o controle de atuação, leitura e manipulação dos dados de leitura, controle das teclas e do display e comunicação com o computador é feito por um microcontrolador PIC18F452 fabricado pela empresa Microchip ${ }^{\circledR}$. Para possibilitar a comunicação com o computador através do padrão RS-232, utilizou-se o componente MAX-232 que faz a interface dos níveis de tensão disponibilizada pelo microcontrolador para os níveis de tensão da porta serial do computador. Foi desenvolvido um circuito analógico com o objetivo de estimular o módulo de alta tensão do sistema com uma tensão senoidal em uma freqüência de $49 \mathrm{kHz}$ e a interface entre o circuito analógico e o controle digital do sistema é responsabilidade de um potenciômetro digital MCP41050IP, cujo valor de resistência total é de $50 \mathrm{k} \Omega$, também fabricado pela Microchip ${ }^{\circledR}$. O circuito de realimentação de tensão é constituído de uma rede resistiva de $100 \mathrm{M} \Omega$ e a realimentação de corrente possui cinco diferentes resistores de Shunt que são selecionados separadamente de acordo com a escala de corrente selecionada. 


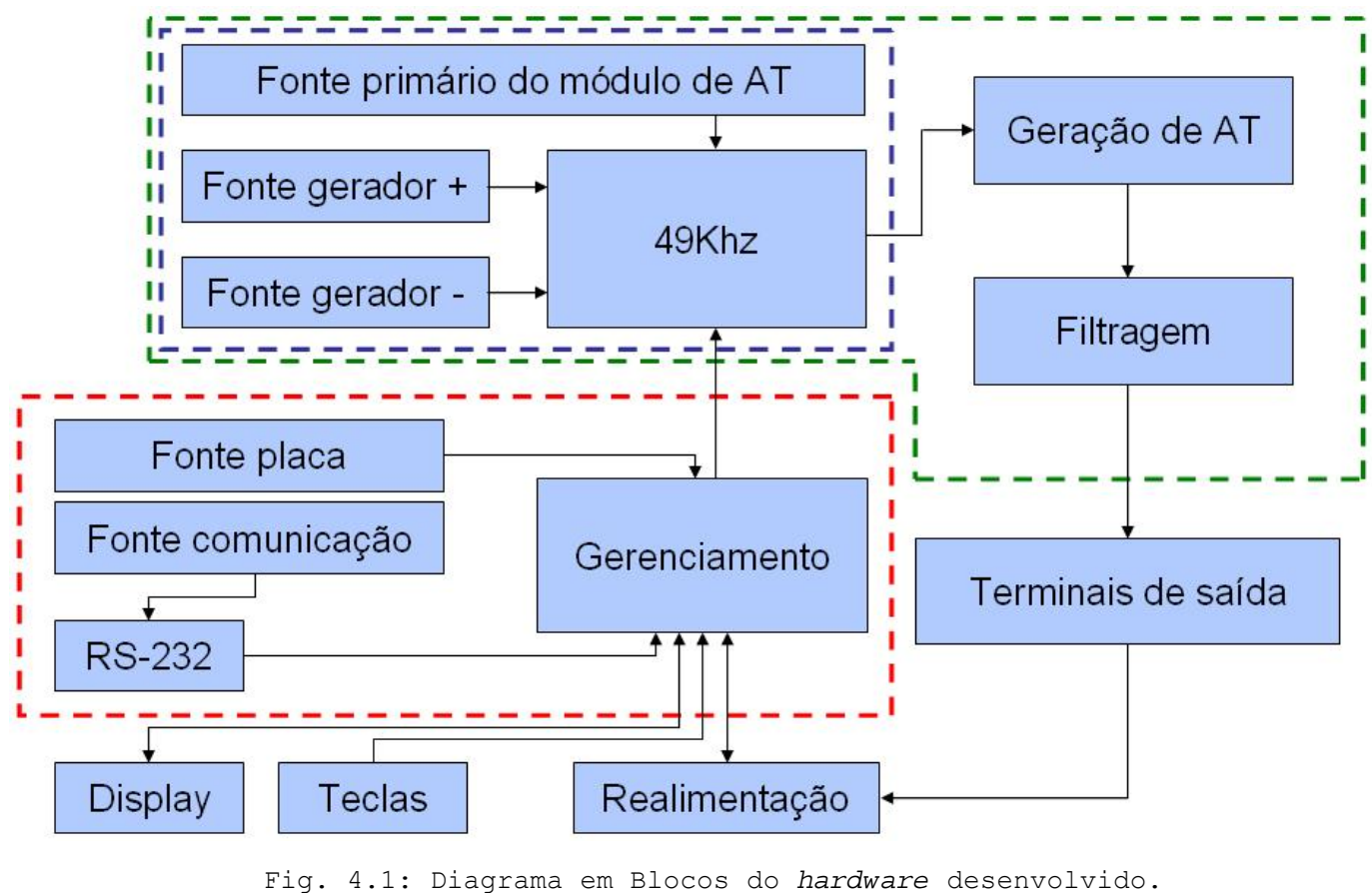

O microcontrolador possui uma programação que foi desenvolvida em um ambiente de programação disponibilizada pelo próprio fabricante do microcontrolador, a Microchip, para download gratuito em sua página na Internet. Esse programa é denominado MPLAB-IDE e a versão que foi utilizada é a versão 7.60. Nele, são elaborados programas para os microcontroladores da linha disponibilizada pela Microchip e utiliza como linguagem padrão o Assembler. No desenvolvimento do software para o sistema objeto desse trabalho, foi instalado um compilador de linguagem " $C$ " e o programa foi elaborado seguindo essa linguagem. Foram utilizadas na programação do microcontrolador, configurações de pinos de I/O, interrupções, além de periféricos como o de comunicação serial USART, conversor analógico digital, comunicação serial SPI, entre outros.

Durante o desenvolvimento, tinha-se como parâmetro fundamental que houvesse a possibilidade de a Fonte de Alta Tensão poder ser operada independentemente da conexão com o computador. Para tal, foi adicionado à Fonte de Alta Tensão, um módulo de Teclas no qual é possível a inserção de todos os parâmetros necessários para o seu funcionamento. A partir daí, os resultados dos ensaios podem ser acompanhados e anotados através das informações contidas no display da Fonte de Alta Tensão. Isso também permite que as condições de emissão de micropontas durante um ensaio não sejam alteradas mesmo que ocorra algum problema de comunicação com o computador. 


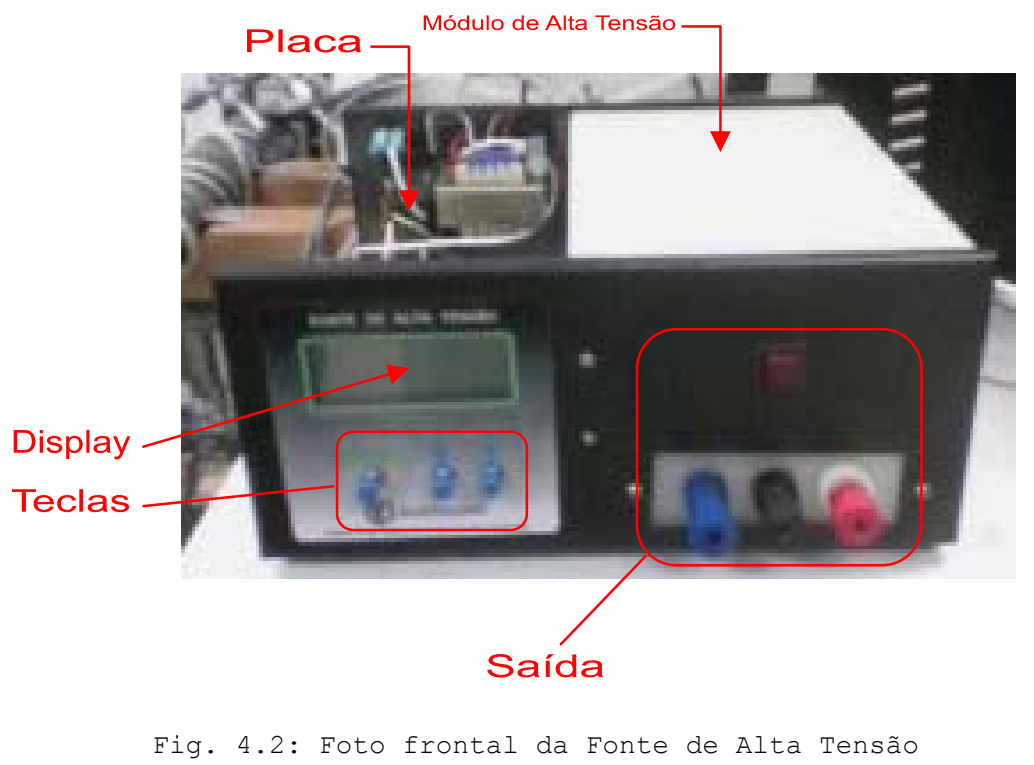

\subsubsection{MÓDULO DE CONTROLE}

Módulo onde se localiza todo o circuito de controle e monitoramento do funcionamento da Fonte de Alta Tensão. Este módulo possui a característica que ter como centro de processamento das informações e da atuação, o microcontrolador fabricado pela microchip modelo PIC18F452. O módulo de controle possui blocos que são responsáveis pela geração de tensão primária, bloco responsável pelo envio e recebimento de dados na comunicação FONTE-PC, bloco responsável por atuar diretamente no circuito primário do módulo de alta tensão e blocos responsáveis por informar o microcontrolador sobre o funcionamento da fonte, através de circuitos de realimentação, além de um módulo de alta tensão onde, ao se aplicar uma tensão no primário, ele impõe uma alta tensão correspondente na saída. Para que o controle seja possível, é necessário atuar diretamente na parte de baixa tensão para que não ocorram problemas na manipulação de altos valores de Tensão. O módulo de controle foi subdividido de acordo com a Figura 4.4: 


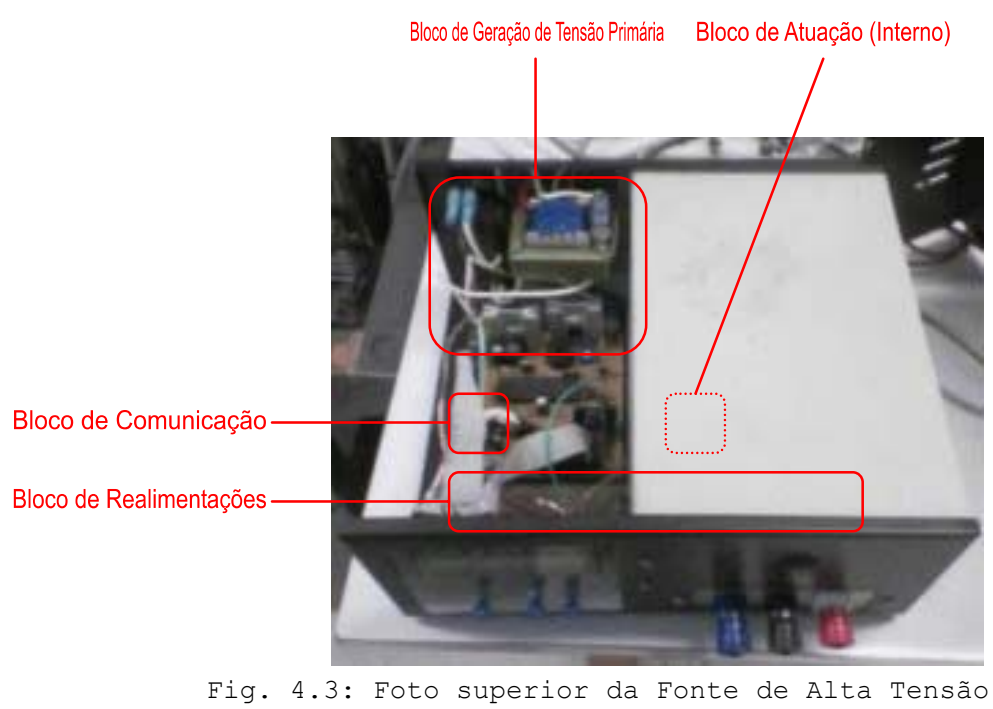

\subsubsection{BLOCOS DE GERAÇÃO DE TENSÃO PRIMÁRIA}

A Fonte de Alta Tensão possui quatro fontes internas para o seu funcionamento, onde as referências são detalhadas na Fig. 4.5. Fonte 1: Fonte responsável por alimentar o circuito do módulo de controle. Essa fonte alimenta com $5 \mathrm{Vdc}$ o circuito que engloba principalmente o microcontrolador. Essa fonte não tem a sua referência "gnd" no mesmo potencial da massa, ou seja, não está em curto com a carcaça. Fonte 2: Fonte responsável por alimentar o circuito de comunicação da placa com o PC. Essa fonte teve que ser feita separadamente para que a referência "gnd" possa ser a massa física da fonte, pois na maioria dos casos os cabos de comunicação serial possuem essa conexão da referência do cabo "gnd" conectado junto à massa "carcaça". Fonte 3 e 4: Fontes responsáveis por impor a tensão de excitação do primário do módulo de alta tensão. Nela, o nível de tensão é controlado através da atuação de um potenciômetro digital que está conectado ao circuito de realimentação do regulador de tensão chaveado "LM2575-ADJ" e que é controlado pelo microcontrolador, essas fontes alimentam também o circuito analógico responsável por gerar a uma onda senoidal na freqüência de $49 \mathrm{kHz}$ e pelos estágios de amplificação. Essa fonte não tem a referência "gnd" no mesmo potencial da massa, ou seja, não está em curto circuito com a carcaça. 


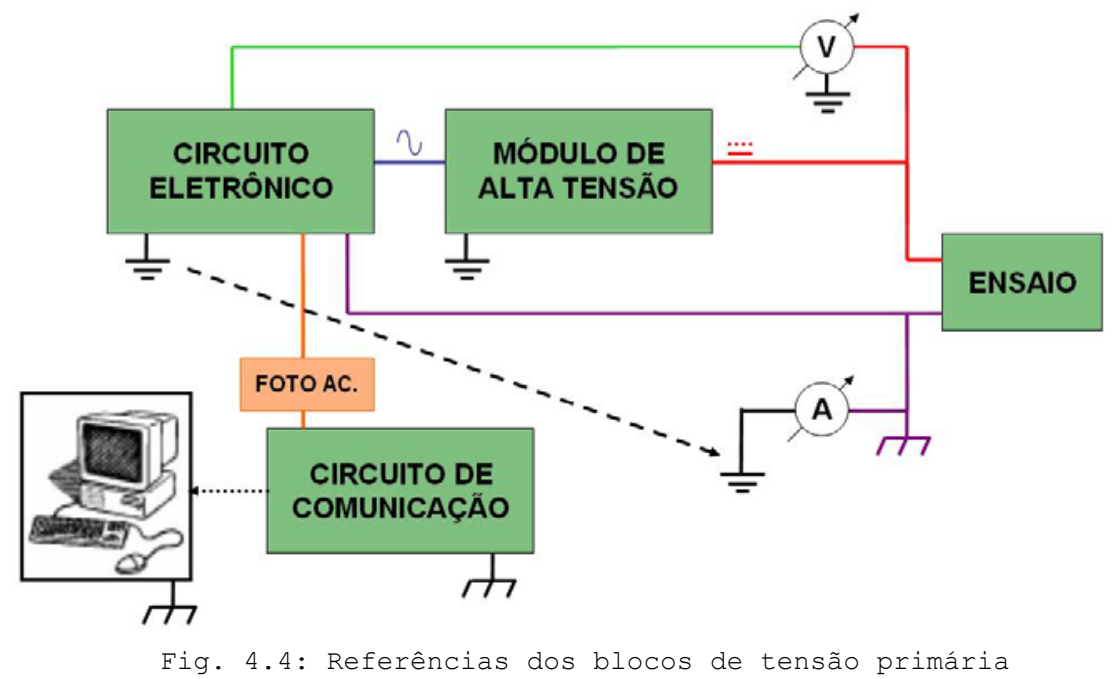

\subsubsection{BLOCO DE COMUNICAÇÃO}

A Fonte de Alta Tensão possui um bloco responsável pelo envio e recebimento de informações da comunicação serial. Esse circuito é alimentado através de uma fonte exclusiva, pois ela possui a referência "gnd" no mesmo potencial da massa, ou seja, está conectada à carcaça da fonte (Fig. 4.5). A partir daí, o sinal chega ao microcontrolador através de um isolamento óptico (fotoacoplador PS2501-1) da mesma forma que o sinal do microcontrolador chega ao MAX-232 também isolado opticamente. Essa isolação é necessária, pois a tensão de alimentação do microcontrolador não está referenciada à carcaça da Fonte de Alta Tensão. Um ponto negativo desse artifício é que limita um pouco a banda (em torno de 9600kbps), porém suficiente para essa aplicação.

\subsubsection{BLOCO DE ATUAÇÃO}

A Fonte de Alta Tensão possui um bloco responsável pela atuação do circuito primário do módulo de Alta Tensão. Esse circuito excita o primário do módulo de Alta Tensão através de um transistor NPN display que recebe um sinal senoidal na freqüência de $49 \mathrm{kHz}$ que foi gerado e amplificado anteriormente. A interação com o módulo de potência, foi feita através de amplificador operacional num arranjo de amplificador de potência (Figura 4.5): 


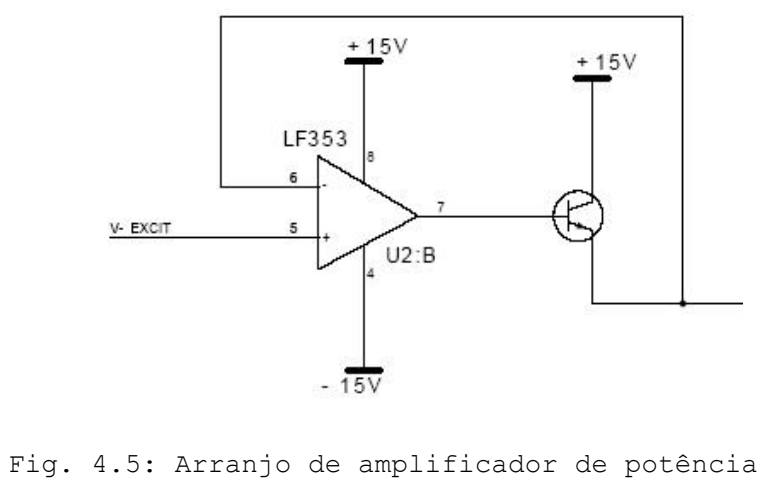

Circuito do Módulo de Alta Tensão: É constituído basicamente de um resistor em série com o módulo (Fig. 4.7), cuja função se caracteriza por limitar a corrente no circuito primário de excitação do módulo que conseqüentemente irá circular pelo transistor de atuação:

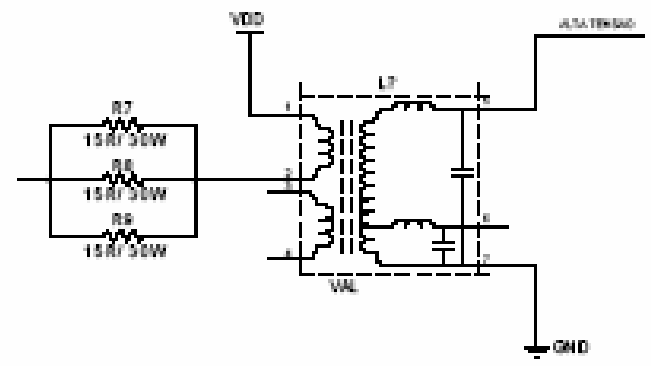

Fig. 4.6: Circuito do Módulo de Alta Tensão.

\subsubsection{BLOCO DE REALIMENTAÇÕES}

A Fonte de Alta Tensão possui dois circuitos de realimentação, um para realimentação de tensão e outro para realimentação de corrente. Circuito de Realimentação de Tensão: É uma associação série de 10 resistores de $10 \mathrm{M} \Omega$ além de um trimpot para calibração. Esse circuito tem a função de dispor no terminal central do trimpot uma tensão proporcional ao valor da tensão de saída. Foi dimensionado para que tenha o valor de 5Vdc no terminal central do potenciômetro quando a tensão de saída for de $10 \mathrm{kVdc}$. Na Figura 4.8, é mostrado o circuito de realimentação de tensão, cada resistência representando, porém dois resistores na montagem real. 


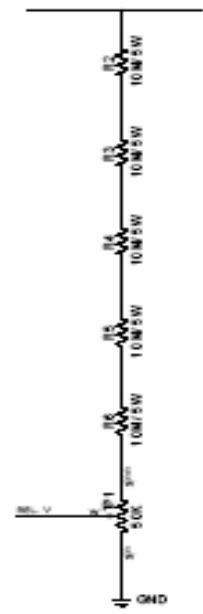

Fig. 4.7: Circuito de Realimentação de Tensão

Circuito de Realimentação de Corrente: É composto de cinco escalas acionadas através de transistores FET de baixa potência como mostra a figura a seguir:

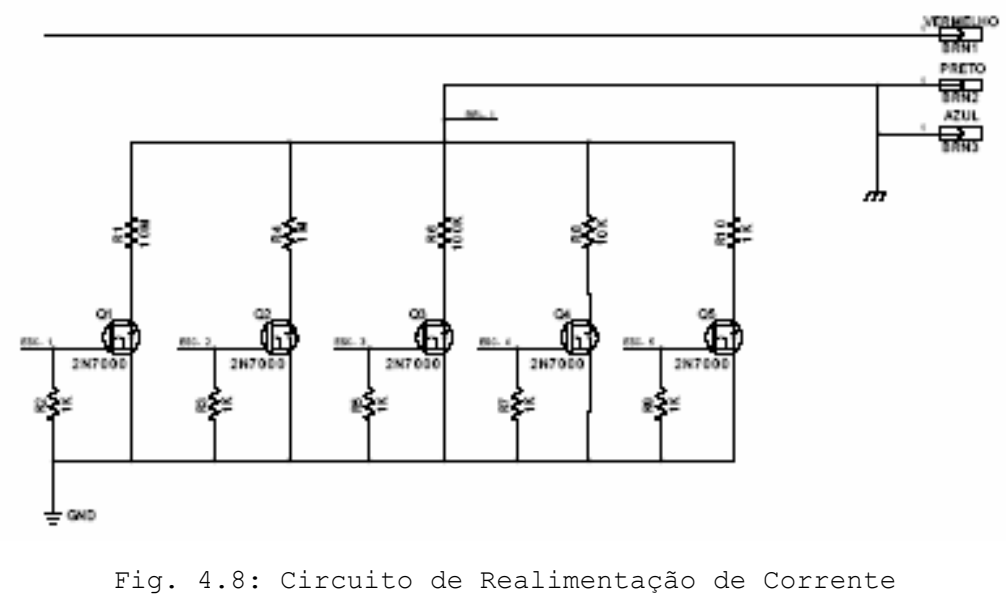

Note que o terminal negativo da fonte de alta tensão está referenciado à "massa" (carcaça da fonte) e o circuito de realimentação de corrente está referenciado ao "gnd" da fonte que é diferente da "massa". A saída da fonte ser referenciada à massa foi uma exigência para a aplicação e caracterização dos dispositivos emissores de campo, porém como pode-se verificar no circuito da Figura 4.9, a saída de alta tensão do módulo está referenciada ao "gnd" da fonte e não à "massa", com isso a circulação de corrente passa através do circuito de realimentação e, conseqüentemente, faz com que ao circular uma corrente através 
do circuito de realimentação de corrente, tenha-se um nível de tensão no ponto "REL-I" do esquema proporcional ao valor da corrente que está circulando.

Foram usados nesse circuito, cinco resistores nos valores de $1 \mathrm{k} \Omega, 10 \mathrm{k} \Omega$, $100 \mathrm{k} \Omega 1 \mathrm{M} \Omega$ e $10 \mathrm{M} \Omega$. Esses resistores determinam uma faixa para cada uma das escalas, como segue a Tabela 4.1:

\begin{tabular}{|c|c|}
\hline \multicolumn{2}{|c|}{ TABELA DE REALIMENTAÇÃO DE CORRENTE } \\
\hline \multirow{2}{*}{ RESISTÊNCIA } & FAIXA DE CORRENTE \\
& \\
\hline $10 \mathrm{M} \Omega$ & $0 \sim 500 \mathrm{nA}$ \\
\hline $1 \mathrm{M} \Omega$ & $0 \sim 5 \mu \mathrm{A}$ \\
\hline $100 \mathrm{k} \Omega$ & $0 \sim 50 \mu \mathrm{A}$ \\
\hline $10 \mathrm{k} \Omega$ & $0 \sim 500 \mu \mathrm{A}$ \\
\hline $1 \mathrm{k} \Omega$ & $0 \sim 5 \mathrm{~mA}$ \\
\hline
\end{tabular}

Tabela 4.1 Escalas da Realimentação de Corrente

\subsubsection{SOFTWARE - HIGHVOLT CONTROLLER}

O software desenvolvido para o computador que incorpora o sistema foi denominado de "HighVolt Controller" (Fig. 4.9) e foi elaborado no ambiente de programação visual orientada a objeto Delphi 7.0 produzido pela empresa Borland. O HighVolt Controller é responsável por controlar a fonte de alta tensão e retirar da fonte todas os parâmetros necessários para apresentar no computador as informações durante a operação. O software desenvolvido apresenta os seguintes recursos: 


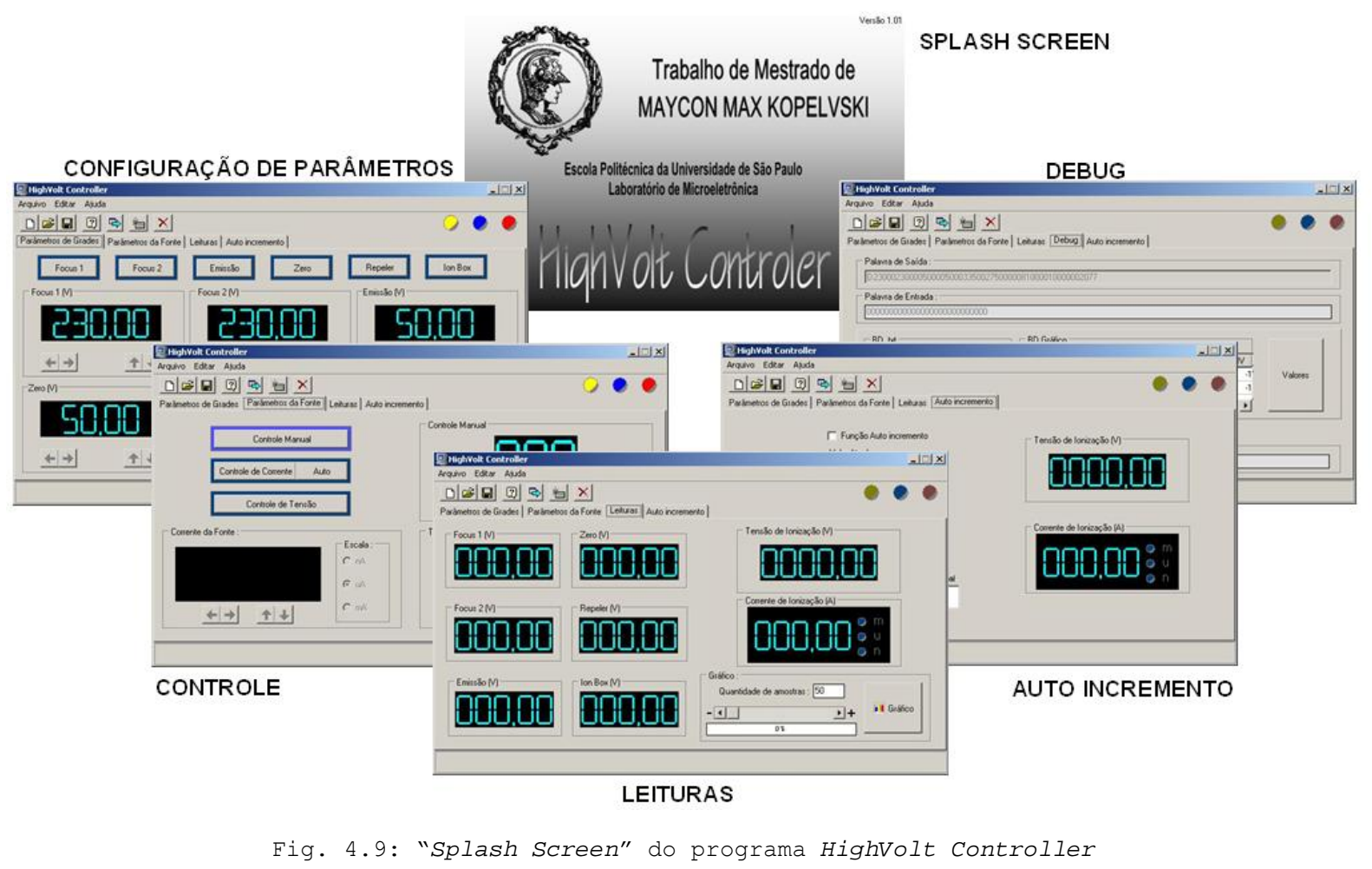

○ Configurar os parâmetros da fonte;

- Configurar os parâmetros das grades de um analisador;

○ Transferir os parâmetros configurados para a fonte, antes ou durante o funcionamento;

○ Gravar os parâmetros configurados;

○ Recuperar os parâmetros que foram gravados anteriormente;

- Acionar e desacionar a porta de comunicação com a fonte;

- Ligar e desligar a fonte sem entrar em contato com a fonte;

- Selecionar qual a porta de comunicação será utilizada;

- Analisar a comunicação no processo de Debug

- Selecionar três modos de operação da fonte: Controle Manual, Controle de Corrente e Controle de Tensão;

- Visualizar as leituras durante o funcionamento;

- Selecionar a velocidade de atualização do gráfico e dos displays indicadores;

○ Visualizar cinco tipos de gráficos: Corrente - Tempo, Tensão - Tempo, Tensão - Corrente, Tensão - Tempo e Corrente - Tempo simultaneamente e Gráfico Fowler-Nordheim; 
- Selecionar a quantidade de amostras para visualização dos Gráficos;

- Exportar os dados armazenados durante todo o ensaio;

\subsubsection{PROTOCOLO DE COMUNICAÇÃO PC - FONTE}

Para a comunicação entre o PC e a Fonte foi desenvolvido um protocolo de transmissão para que a fonte pudesse interpretar o pacote de dados transmitido. Com isso, os pacotes transmitidos foram padronizados de duas formas:

$\begin{array}{ll}\checkmark & \text { Pacote de dados; } \\ \checkmark & \text { Pacote de instrução. }\end{array}$

No pacote de instrução, a informação é composta de cinco caracteres que são transmitidos de acordo com o padrão ASCII. O pacote de instrução a ser transmitido é construído com o seguinte critério:

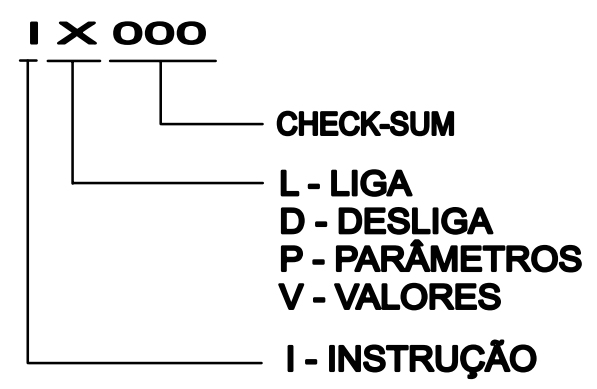

Os pacotes de instrução são apresentados a seguir:

IL149 - Instrução liga. Essa instrução é transmitida para a fonte sempre que é solicitado através do software que a fonte ligue.

ID141 - Instrução desliga. Essa instrução é transmitida para a fonte sempre que é solicitado através do software que a fonte desligue.

IP153 - Instrução parâmetro. Essa instrução é transmitida para a fonte sempre que é solicitado que seja transferido para a fonte os parâmetros configurados no software.

IV159 - Instrução valores. Essa instrução é transmitida para a fonte sempre que o software atualizar os gráficos e os dados do experimento. Essa instrução é transmitida somente quando a fonte está ligada e a repetição dessa instrução varia de acordo com o parâmetro de velocidade de varredura configurada no software. 
No pacote de dados, a informação é composta de 51 caracteres que são transmitidos de acordo com o padrão ASCII. O pacote de dados a ser transmitido é construído seguindo o seguinte critério:

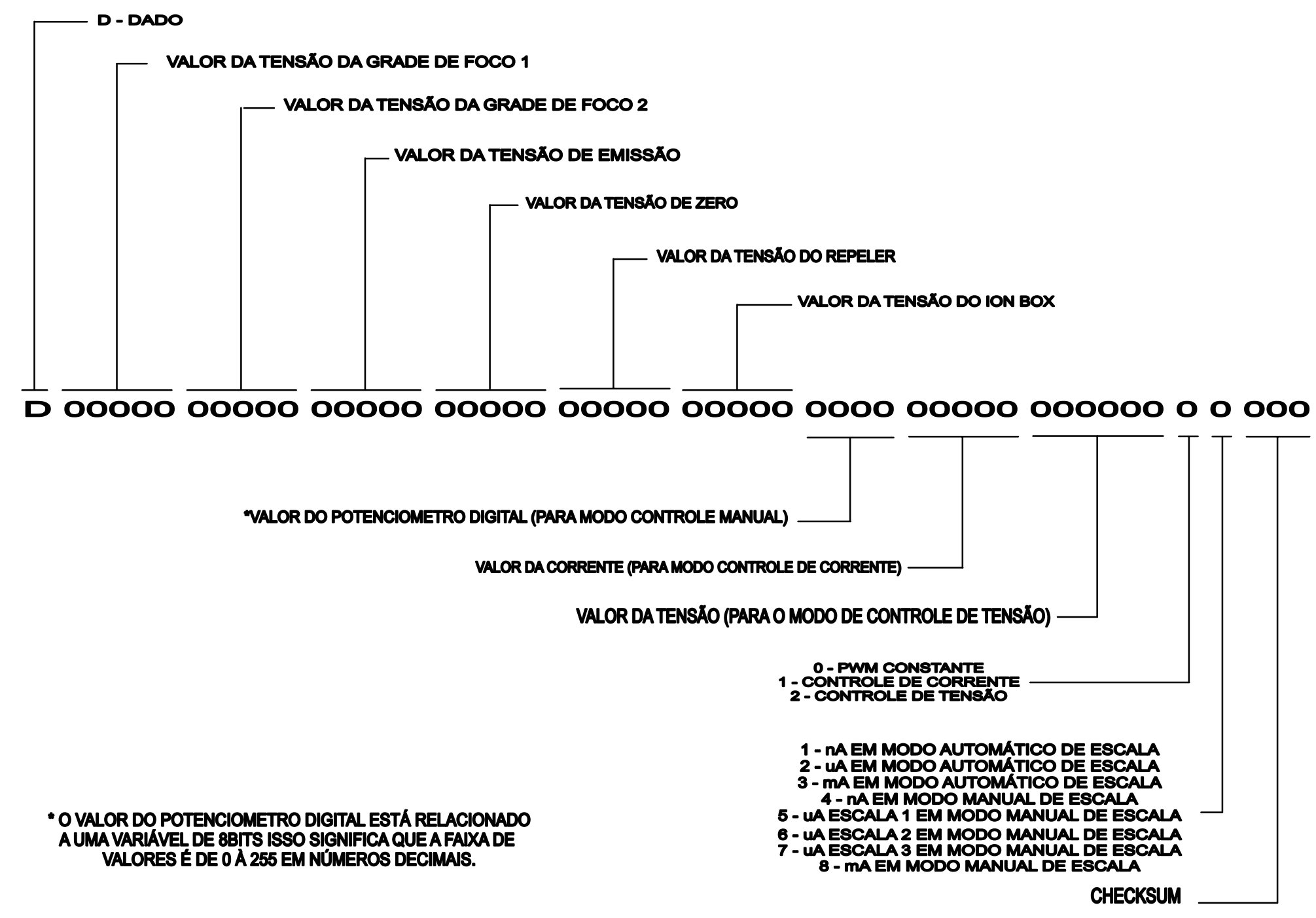




\subsubsection{CHECKSUM}

Com o objetivo de aceitar somente as mensagens que não contenham erros, foi aplicada uma forma de detecção de erros baseada no conceito de checksum. Um código é gerado a partir do tratamento da palavra a ser transmitida, calculando byte a byte e sem aplicar nenhuma equação relacionada a CRC (Ciclic Redundance Check). A metodologia aplicada para esse cálculo é a de somar todos os bytes da palavra a ser transmitida sem considerar o carry, o resultado nunca ultrapassa 8 bits:

exemplo 1:

- INSTRUÇÃO LIGA:

CARACTER: I $\quad($ CÓDIGO ASCII $=73 \mathrm{~d})$

+ L $($ CÓDIGO ASCII = 76d $)$

149 (Checksum)

PALAVRA: IL149

exemplo 2:

- INSTRUÇÃO VALORES:

CARACTER: I $\quad($ CÓDIGO ASCII = 73d $) \quad 01001001 \mathrm{~b}$

$\underline{+\mathrm{V}}($ CÓDIGO ASCII = 86d $) \quad \underline{+01010110 \mathrm{~b}}$

159 (Checksum) $10011111 \mathrm{~b}$

PALAVRA: IV159

exemplo 3:

- DADOS:

Um exemplo do cálculo do pacote de dados foi colocado como anexo (Anexo 8.9).

Foi escolhida essa forma de cálculo de Checksum, pois como em alguns momentos quem calcula o Checksum é o microcontrolador, não se desejava sobrecarregá-lo com instruções complexas e aumentar muito a quantidade de memória usada para esse cálculo. 
Neste projeto, não se visava contabilizar toda palavra recebida, porém simplesmente ignorar em alguns casos ou enviar uma palavra de erro para que o computador pudesse reenviar a palavra.

Existe, porém a probabilidade de em casos raros, serem contabilizadas palavras erradas, mas com Checksum corretos, pois palavras diferentes podem ter o mesmo Checksum. Isso se deve ao equacionamento de natureza limitada do Checksum.

\subsection{SOFTWARE}

\subsubsection{ESTRUTURA DO HIGHVOLT CONTROLLER}

O programa HighVolt Controller foi desenvolvido de acordo com a estrutura apresentada no Anexo 8.8. Dessa estrutura, os seguintes itens destacam-se:

\subsubsection{JANELA PRINCIPAL - PASTAS - PARÂMETROS DE USO GERAL}

Nessa pasta, é possível configurar alguns parâmetros de uso geral, essa pasta é destinada para futuras aplicações do sistema, onde for necessário o controle - através da atuação e leitura - de outros fatores envolvidos nos ensaios. As informações configuradas nos parâmetros de uso geral são previstas no protocolo de comunicação PC-FONTE e FONTE-PC desenvolvido.

A extração do valor configurado dos parâmetros de uso geral, no pacote de dados PC-FONTE, permite a atuação em circuitos externos que venham a ser desenvolvidos no futuro. E a adição dos valores medidos no pacote de dados FONTE-PC, faz com que esses valores sejam indicados e acompanhados através dos displays da pasta Leituras.

Para que essa função seja corretamente utilizada, é necessário que a implementação siga os critérios determinados no protocolo, onde cada informação tem a sua posição correta para a extração bem como para a inserção dos dados.

\subsubsection{JANELA PRINCIPAL - PASTAS - PARÂMETROS DA FONTE}

Nessa pasta, é possível configurar os parâmetros da Fonte de Alta Tensão.

Botão Controle Manual: Ao selecionar-se essa opção, o Bloco de Configuração de Controle Manual é ativado, dessa forma, pode-se através dos botões direcionais direita e esquerda, selecionar o dígito que se deseja alterar e através dos botões direcionais para 
cima e para baixo, incrementar ou decrementar o dígito selecionado anteriormente. Nesse modo de funcionamento, a Fonte de Alta Tensão não fará nenhum tipo de controle (Controle de Tensão ou Controle de Corrente), com isso o valor medido de tensão poderá cair, caso o consumo de corrente aumente. Nesses casos, o usuário deverá interferir manualmente através da transmissão de um novo parâmetro ou interferindo através dos botões localizados no painel da Fonte. Nota: Os parâmetros são alterados livremente, desde que dentro da faixa de valores aceitável.

Botão Controle de Corrente: Ao selecionar-se essa opção, o Bloco de Controle de Corrente é ativado, dessa forma, pode-se através dos botões direcionais direita e esquerda, selecionar o dígito que se deseja alterar e através dos botões direcionais para cima e para baixo, incrementar ou decrementar o dígito selecionado anteriormente, além de possibilitar, selecionar através da caixa combo qual a escala de corrente desejada. Nesse modo de funcionamento, a Fonte de Alta Tensão manterá constantemente o valor mais próximo do valor configurado de Corrente no software. Nota: Os parâmetros são alterados livremente, desde que dentro da faixa de valores aceitável.

Botão Escala de Medição: Ao selecionar-se essa opção, se escolhe qual a escala do medidor de corrente da Fonte de Alta Tensão. Conforme é pressionado, o texto do botão é alterado e passa a ter o valor da escala selecionada, conforme a Tabela 4.2:

Botão Controle de Tensão: Ao selecionar-se essa opção, o Bloco de Controle de Tensão é ativado, dessa forma, pode-se através dos botões direcionais direita e esquerda, selecionar o dígito que se deseja alterar e através dos botões direcionais para cima e para baixo, incrementar ou decrementar o dígito selecionado anteriormente. Nesse modo de funcionamento, a Fonte de Alta Tensão manterá constantemente o valor mais próximo do valor configurado de Tensão no software. Nota: Os parâmetros são alterados livremente, desde que dentro da faixa de valores aceitável. 


\begin{tabular}{|c|c|}
\hline Modo & Descrição \\
\hline Auto & $\begin{array}{l}\text { A Fonte de Alta Tensão busca a Escala } \\
\text { automaticamente de acordo com o valor medido... }\end{array}$ \\
\hline $\operatorname{Man}(\mathrm{nA})$ & $\begin{array}{l}\text { A Fonte de Alta Tensão, passa a acionar o circuito de } \\
\text { leitura de corrente com o modo manual, na escala de } \\
\text { nA. Essa escala, indica leituras na faixa de } 0 \text { à } 500 \\
\text { nA. }\end{array}$ \\
\hline $\operatorname{Man}(\mu \mathrm{A}-1)$ & $\begin{array}{l}\text { A Fonte de Alta Tensão, passa a acionar o circuito de } \\
\text { leitura de corrente com o modo manual, na escala de } \\
\mu \mathrm{A} \text {. Essa escala, indica leituras na faixa de } 0 \text { à } 5 \mu \mathrm{A} \text {. } \\
\text { Porém a sua utilização é indicada nos casos de } \\
\text { leituras na faixa de } 500 \mathrm{nA} \text { à } 5 \mu \mathrm{A}\end{array}$ \\
\hline $\operatorname{Man}(\mu \mathrm{A}-2)$ & $\begin{array}{l}\text { A Fonte de Alta Tensão, passa a acionar o circuito de } \\
\text { leitura de corrente com o modo manual, na escala de } \\
\mu \mathrm{A} . \text { Essa escala, indica leituras na faixa de } 0 \text { à } 50 \\
\mu \mathrm{A} \text {. Porém a sua utilização é indicada nos casos de } \\
\text { leituras na faixa de } 5 \mu \mathrm{A} \text { à } 50 \mu \mathrm{A}\end{array}$ \\
\hline $\operatorname{Man}(\mu \mathrm{A}-3)$ & $\begin{array}{l}\text { A Fonte de Alta Tensão, passa a acionar o circuito de } \\
\text { leitura de corrente com o modo manual, na escala de } \\
\mu \mathrm{A} . \text { Essa escala, indica leituras na faixa de } 0 \text { à } 500 \\
\mu \mathrm{A} \text {. Porém a sua utilização é indicada nos casos de } \\
\text { leituras na faixa de } 50 \mu \mathrm{A} \text { à } 500 \mu \mathrm{A}\end{array}$ \\
\hline $\operatorname{Man}(\mathrm{mA})$ & $\begin{array}{l}\text { A Fonte de Alta Tensão, passa a acionar o circuito de } \\
\text { leitura de corrente com o modo manual, na escala de } \\
\mu \mathrm{A} \text {. Essa escala, indica leituras na faixa de } 0 \text { à } 5 \mathrm{~mA} \text {. } \\
\text { Porém a sua utilização é indicada nos casos de } \\
\text { leituras na faixa de } 500 \mu \mathrm{A} \text { à } 5 \mathrm{~mA}\end{array}$ \\
\hline
\end{tabular}

Tabela 4.2 Escalas de Medição de Corrente

\subsubsection{JANELA PRINCIPAL - PASTAS - DEBUG}

Nessa ficha, é possível analisar a comunicação com a fonte, além de monitorar os banco de dados amostrados armazenados para exportação e o banco de dados amostrados e armazenados para a geração dos gráficos as palavras de comunicação são atualizadas "on-line" durante o funcionamento da Fonte de Alta Tensão, dessa forma, podem ser 
monitorados durante os ensaios. Nota: Essa ficha só é ativada, caso o Menu Editar > Debugar estiver em "SIM". A seguir são descritas as informações encontradas nessa pasta:

Palavra de Saída: O texto contido nessa caixa indica a palavra que será transmitida para a Fonte de Alta Tensão quando for solicitada a transferência de Parâmetros.

Palavra de Entrada: O texto contido nessa caixa indica a palavra que foi recebida pela fonte via comunicação serial e aceita, pois essa caixa só discrimina as palavras que já passaram pela checagem do Checksum.

BD .txt: $O$ texto contido nessa caixa é o banco de dados que será exportado quando for solicitado a gravação dos dados do ensaio com um arquivo com uma extensão .txt (texto).

Indicador de Gravação: O texto indicado mostra se os parâmetros configurados já foram gravados ou se são um novo parâmetro, caso já tenham sido gravados, será indicado o caminho completo do local onde o arquivo de parâmetros foi gravado.

Recebido fonte: $O$ texto contido nessa caixa indica a palavra que foi recebida pela fonte via comunicação serial, essa caixa discrimina as palavras mesmo sem a checagem do Checksum.

BD Gráfico: A tabela contida nessa caixa indica as amostras que estão sendo indicadas no gráfico quando forem visualizados.

Indicador de Amostra: O texto indicado mostra o controle de amostras efetuadas nos ensaios.

Botão Valores: Ao ser pressionado esse botão, o software envia uma solicitação via serial, para que a Fonte de Alta Tensão transmita os valores de leitura.

\subsubsection{JANELA PRINCIPAL - PASTAS - AUTO-INCREMENTO}

Nessa ficha, é possível configurar o SCEDEC para que execute automaticamente um procedimento de caracterização através do incremento da tensão da fonte sem a interação do usuário. Nele, o usuário apenas determina quais serão os parâmetros do ensaio e a partir desse momento, o sistema assume a troca dos parâmetros e recolhimento dos valores medidos. A seguir são descritas as informações encontradas nessa pasta:

Check Auto-incremento: Ao selecionar esse campo, o usuário estará habilitando a função auto-incremento. 
Display Valor Atual: Nesse display, é indicado qual é o valor atual configurado do potenciômetro digital que o sistema está implementando naquele momento do ensaio.

Display Tensão da Fonte: Nesse display, é indicado "on-line" qual é o valor da tensão da fonte.

Display Corrente Consumida: Nesse display, é indicado "on-line” qual é o valor da corrente elétrica da fonte, que está sendo consumida.

“Text Box" Segundos: Nesse espaço, o usuário insere o valor, em segundos, que o incremento do valor da tensão da fonte deverá ser feito pelo sistema.

"Text Box" Valor inicial: Nesse espaço, o usuário insere o valor inicial, de acordo com a faixa de valores do potenciômetro digital $(0-255)$, que o ensaio deverá ser iniciado.

"Text Box" Valor final: Nesse espaço, o usuário insere o valor final, de acordo com a faixa de valores do potenciômetro digital $(0-255)$, que o incremento de tensão deverá parar.

\subsubsection{JANELA GRÁFICOS}

Ao se pressionar o botão Gráficos na pasta Leitura na Janela Principal, será aberta uma Janela que mostrará os gráficos durante os ensaios. O software possui cinco tipos gráficos que são atualizados "on-line" durante os ensaios, não eles:

- Gráfico I-t;

- Gráfico V-t;

- Gráfico V-I;

- Gráfico Duplo (I-t e V-t simultaneamente), e;

- Gráfico de Fowler-Nordheim.

Gráfico I-t - Indica o comportamento da corrente em função do tempo;

Gráfico V-t - Indica o comportamento da tensão em função do tempo;

Gráfico V-I - Indica o comportamento da tensão em função da corrente;

Gráfico F-N - Indica o gráfico de Fowler-Nordheim: $\ln \left(\frac{I}{V^{2}}\right)$ em função de $\frac{1}{V}$.

Gráfico Duplo - Indica o comportamento da tensão em função do tempo e o comportamento da corrente em função do tempo simultaneamente. 


\subsubsection{CARACTERÍSTICAS TÉCNICAS}

As especificações da Fonte de Alta Tensão desenvolvida são apresentadas na Tabela 4.3 a seguir:

\begin{tabular}{|c|c|}
\hline CARACTERISTICA & DESCRIÇÃO \\
\hline \multicolumn{2}{|l|}{ ESTABILIDADE } \\
\hline DISPLAY & 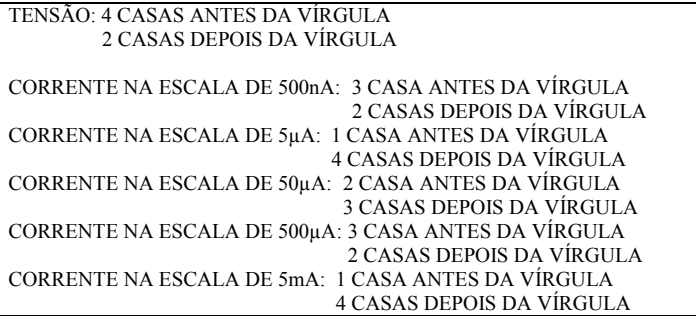 \\
\hline AJUSTE & FAIXA DE AJUSTE (FINO / GROSSO) \\
\hline CIRCUITO DE PROTEÇÃO DE SOBRECARGA & SIM \\
\hline MODOS DE OPERAÇÃO & TENSÃO CONTÍNUA, CONTROLE DE CORRENTE, CONTROLE MANUAL \\
\hline PROTEÇÃO DE INVERSÃO DE POLARIDADE & NÃO \\
\hline ALIMENTAÇÃO & $110 / 220 \mathrm{~V}$ AC $\pm 10 \%, 50 / 60 \mathrm{~Hz}$ SELECIONÁVEL \\
\hline CONSUMO MÁXIMO & $1 \mathrm{~A}$ \\
\hline USO & INTERNO \\
\hline DIMENSÕES & $12,5 \mathrm{~mm}(\mathrm{~A}) \times 28 \mathrm{~mm}(\mathrm{~L}) \times 24,5(\mathrm{P})$ \\
\hline PESO & $5 \mathrm{~kg}$ \\
\hline \multicolumn{2}{|r|}{ OPERAÇÃO TENSÃO CONSTANTE } \\
\hline TENSÃO DE SAÍDA & $1 \sim 4 \mathrm{kV}$ \\
\hline RIPPLE \& RUÍDO & $\sim 5 \%$ DA TENSÃO DE SAÍDA \\
\hline \multicolumn{2}{|r|}{ OPERAÇÃO CORRENTE CONSTANTE } \\
\hline CORRENTE DE SAÍDA & $0 \sim 1 \mathrm{~mA}$ \\
\hline TOLERÂNCIA CORRENTE & $\begin{array}{l}\text { CORRENTE NA ESCALA DE } 500 \mathrm{nA}: \pm 9,873 \% \\
\text { CORRENTE NA ESCALA DE } 5 \mu \mathrm{A}: \pm 2,69 \% \\
\text { CORRENTE NA ESCALA DE } 50 \mu \mathrm{A}: \pm 0,98 \% \\
\text { CORRENTE NA ESCALA DE } 500 \mu \mathrm{A}: \pm 0,88 \% \\
\text { CORRENTE NA ESCALA DE } 5 \mathrm{~mA}: \pm 1,56 \%\end{array}$ \\
\hline \multicolumn{2}{|r|}{ ACESSÓRIOS NECESSÁRIOS } \\
\hline CABO SERIAL & 1 \\
\hline CABO AC & 1 \\
\hline CABO DE CONEXÃO & 2 (PINO BANANA - GARRA JACARÉ) \\
\hline
\end{tabular}

Tabela 4.3 Características Técnicas

\subsubsection{PROTOCOLO DE COMUNICAÇÃO FONTE - PC}

Na comunicação entre a Fonte e o PC, fora seguidos os mesmo critérios da Comunicação PC-FONTE que foi detalhada no item 4.1.3 dessa dissertação, uma vez que como a Fonte se comunica com o PC, a Fonte deve interpretar os pacotes de dados enviados pelo PC. A diferença está no fato de que a Fonte responde para o PC sempre que ela recebe um pacote de dados. Essa resposta discrimina se a informação foi recebida com sucesso ou se houve erro na recepção dos dados, e no caso de receber uma solicitação dos valores que estão 
sendo medido nos ensaios, a Fonte responde com o pacote de dados contendo os valores medidos. Com isso, os pacotes transmitidos foram padronizados de duas formas:

\footnotetext{
$\checkmark \quad$ Pacote de instrução de resposta;

$\checkmark \quad$ Pacote de dados.
}

No pacote de instrução de resposta, a informação é composta de cinco caracteres que são transmitidos de acordo com o padrão ASCII. O pacote de instrução de resposta a ser transmitido é construído com o seguinte critério:

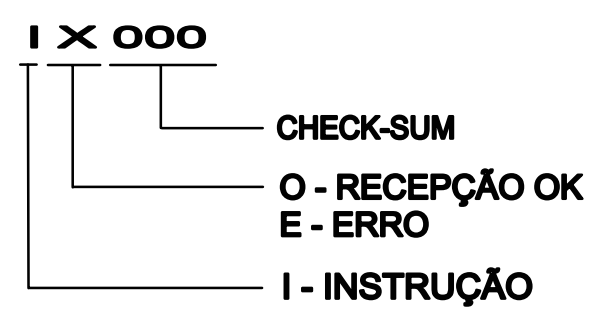

Os pacotes de instrução de resposta são apresentados a seguir:

I0152 - Instrução de recepção OK. Essa instrução é transmitida para o PC sempre que houver uma confirmação na checagem do Checksum após a recepção de um pacote de dados.

IE142 - Instrução de erro. Essa instrução é transmitida para o PC sempre que NÃO houver uma confirmação na checagem do Checksum após a recepção de um pacote de dados. 
No pacote de dados, a informação é composta de 51 caracteres que são transmitidos de acordo com o padrão ASCII. O pacote de dados a ser transmitido é construído seguindo o seguinte critério:

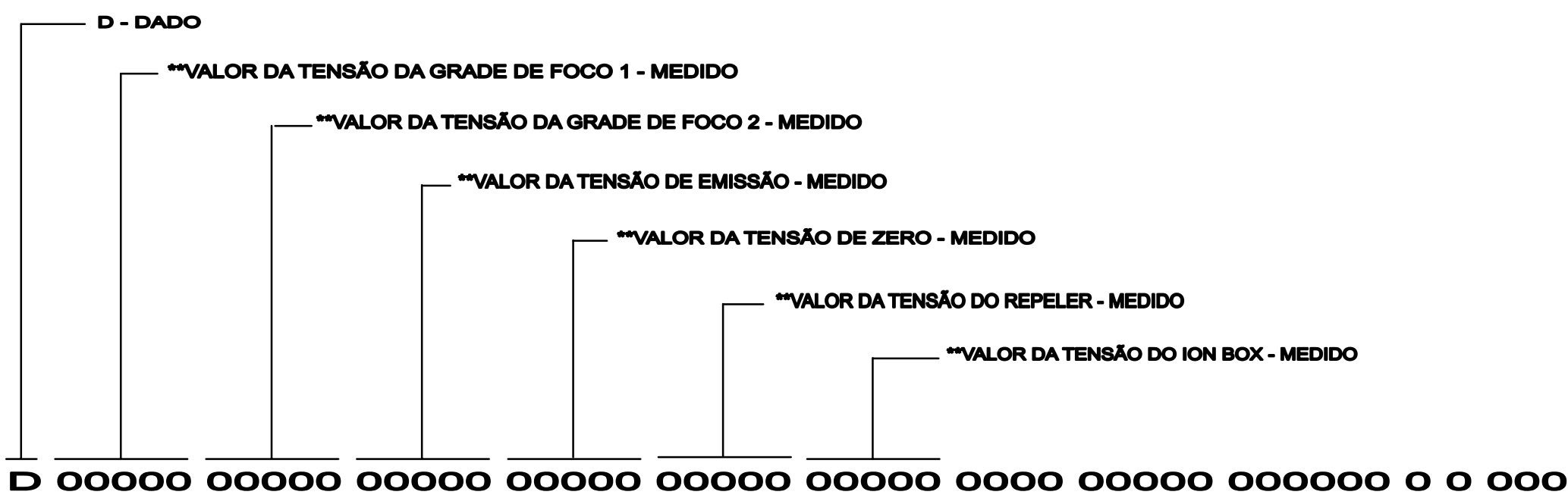

VALOR DO POTENCIOMETRO DIGITAL (PARA MODO CONTROLE MANUAL)

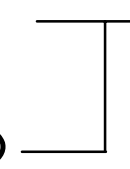

VALOR DA CORRENTE - MEDIDA

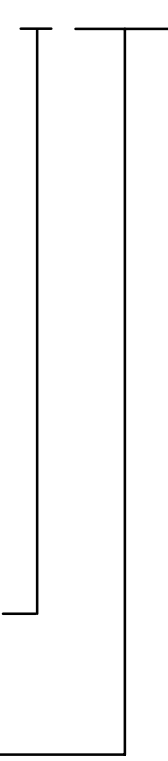


O protocolo de comunicação foi elaborado também visando a possibilidade de futuramente ser utilizado no controle de outros fatores envolvidos nos ensaios. No protocolo FONTE-PC, os valores devem ser inseridos nos locais reservados para cada parâmetro, dessa forma, tais valores serão indicados nos displays da pasta Leituras do programa HighVolt Controller.

\subsection{PUBLICAÇÕES}

Devido ao fato de se verificar uma satisfatória concordância com o aspecto esperado para o comportamento V x I e F-N mostrando que o sistema de caracterização desenvolvido apresenta boa funcionalidade para estudo de FED, foi possível a apresentação de diversos trabalhos do grupo GSIM, são eles:

1) "HI-PS Technology Applied on Si Tips Fabrication for Field Emission Devices" no evento Ibersensor-2006 (DANTAS,2006).

2) "Silicon Microtips Array Fabricated by HI-PS Technique for Application in Field Emission Devices" no evento SBMicro-2007 (DANTAS,2007).

3) "Field Emission Devices Electrical Characteristics Trial System" no evento SForum2007 (KOPELVSKI,2006).

4) "Silicon Field Emission Devices fabricated by HI-PS Technique" no evento SBMat2007 (DANTAS,2007B).

\subsection{CALIBRAÇÃO}

A calibração do SCEDEC foi elaborada seguindo o seguinte critério: A partir de resistências com valores conhecidos, foi possível saber a corrente esperada para uma determinado valor de resistência de carga, com relação a diferença de potencial aplicada sobre essa resistência. A partir desse momento, foi interligado ao sistema dois equipamentos de medição, são eles:

1) Fluke 800A Digital Multimeter. Esse instrumento foi ligado ao circuito de calibração para indicar os valores de tensão;

2) HP 34401 - Multimeter. Esse instrumento foi ligado ao circuito de calibração para indicar os valores de corrente.

O esquema de ligação do SCEDEC à resistência de calibração, bem como os instrumentos de medição, são indicados na Figura. 4.11: 


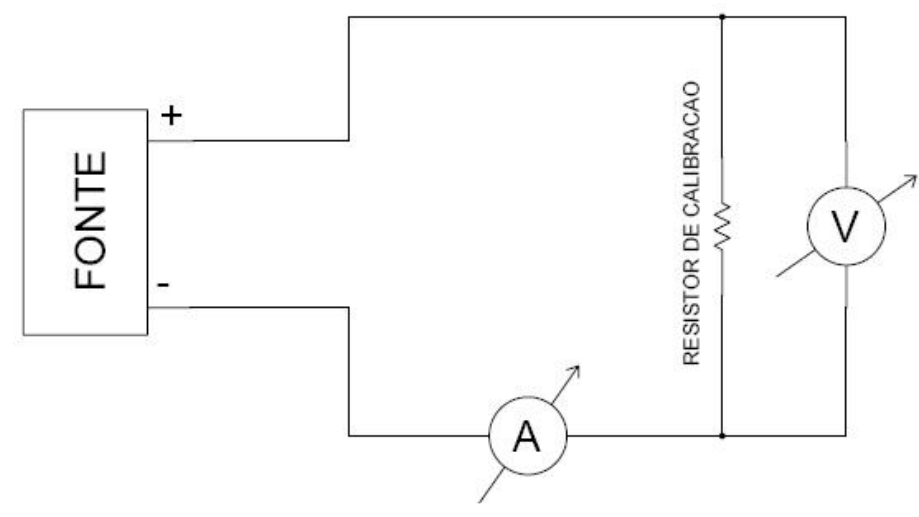

Fig. 4.10: Esquema de ligação do circuito de Calibração

A partir desse momento, o procedimento de calibração foi executado, uma escala de cada vez, sempre tendo como referência a indicação dos medidores

\subsubsection{RESISTORES DE CALIBRAÇÃO}

Para o processo de validação do SCEDEC, foram utilizadas cinco resistências de calibração. Para que essas resistências fossem classificadas como resistências de calibração, foi executado um processo de medição com um "Precision Semiconductor Parameter Analyzer" Modelo HP4156A, com o objetivo de obter com a máxima precisão possível, qual o valor exato que cada resistência apresenta.

\subsubsection{RESISTOR DE 1G $\Omega$}

Para a calibração da escala de 500nA, utilizou-se como resistência de calibração, uma ponta de medição de um multímetro para alta tensão, essa ponta tem como principal característica, um alto valor de resistência equivalente aproximadamente $1 \mathrm{G} \Omega$. O esquema apresentado na Figura 4.12, mostra como é a ligação interna da monta de medição. Os pontos que foram utilizados na calibração foram os pontos B e C.

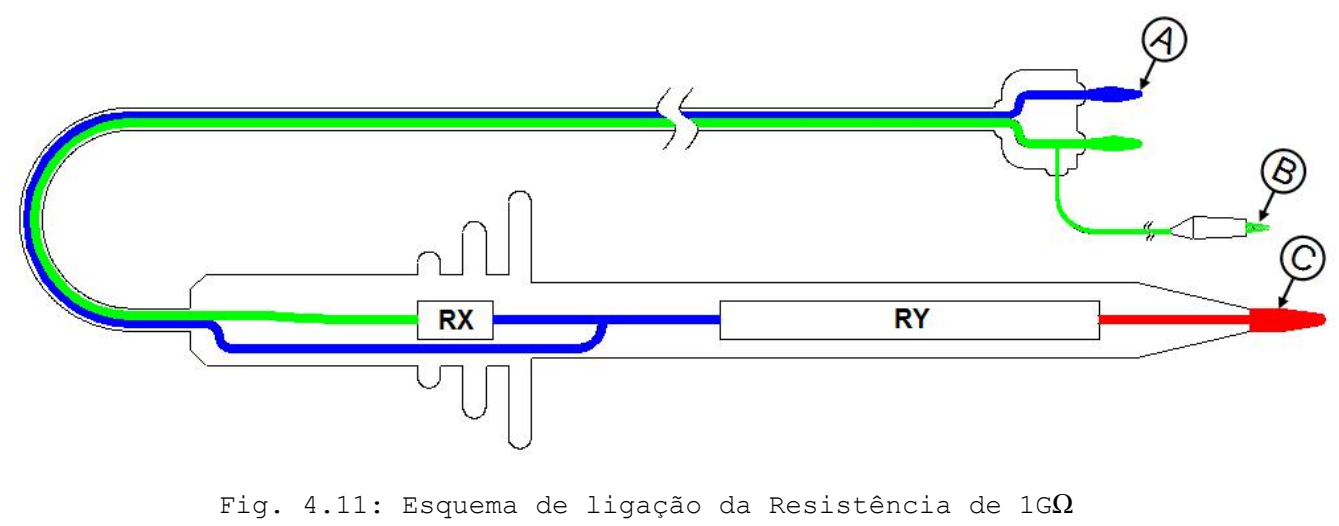


Durante o processo de medição do valor indicado pelo equipamento HP4156A, foi de:

\begin{tabular}{|c|c|}
\hline PONTOS & VALORES \\
\hline B e C & $990 \mathrm{M} \Omega$ \\
\hline
\end{tabular}

\subsubsection{RESISTOR DE 200M $\quad$}

Para a calibração da escala de $5 \mu \mathrm{A}$, utilizou-se como resistência de calibração, uma rede de resistências em série. A rede é formada por 10 resistências cujo valor indicado no corpo, através de código de cores é igual a $20 \mathrm{M} \Omega$, com isso desejava-se obter um valor de resistência equivalente de $200 \mathrm{M} \Omega$. O esquema apresentado na Figura 4.14, mostra como é a ligação para a formação da rede resistiva:

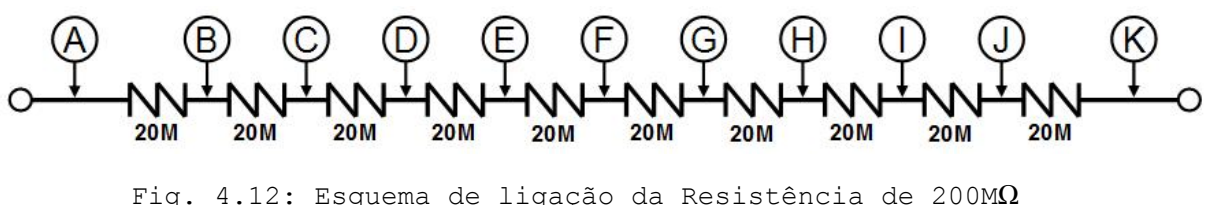

Durante o processo de medição do valor indicado pelo equipamento HP4156A, foi de:

\begin{tabular}{|c|c|}
\hline PONTOS & VALORES \\
\hline AB & $20,535 \mathrm{M} \Omega$ \\
\hline $\mathrm{AC}$ & $41,26 \mathrm{M} \Omega$ \\
\hline $\mathrm{AD}$ & $60,9384 \mathrm{M} \Omega$ \\
\hline $\mathrm{AE}$ & $83,225 \mathrm{M} \Omega$ \\
\hline $\mathrm{AF}$ & $105,196 \mathrm{M} \Omega$ \\
\hline $\mathrm{AG}$ & $127,5 \mathrm{M} \Omega$ \\
\hline $\mathrm{AH}$ & $147,24 \mathrm{M} \Omega$ \\
\hline $\mathrm{AI}$ & $169,5 \mathrm{M} \Omega$ \\
\hline $\mathrm{AJ}$ & $189,7 \mathrm{M} \Omega$ \\
\hline $\mathrm{AK}$ & $211 \mathrm{M} \Omega$ \\
\hline
\end{tabular}




\begin{tabular}{|c|c|}
\hline PONTOS & VALORES \\
\hline AB & $20,535 \mathrm{M} \Omega$ \\
\hline BC & $20,725 \mathrm{M} \Omega$ \\
\hline $\mathrm{CD}$ & $19,6784 \mathrm{M} \Omega$ \\
\hline $\mathrm{DE}$ & $22,2866 \mathrm{M} \Omega$ \\
\hline $\mathrm{EF}$ & $21,971 \mathrm{M} \Omega$ \\
\hline $\mathrm{FG}$ & $22,304 \mathrm{M} \Omega$ \\
\hline $\mathrm{GH}$ & $19,74 \mathrm{M} \Omega$ \\
\hline $\mathrm{HI}$ & $22,26 \mathrm{M} \Omega$ \\
\hline $\mathrm{IJ}$ & $20,2 \mathrm{M} \Omega$ \\
\hline JK & $21,3 \mathrm{M} \Omega$ \\
\hline
\end{tabular}

\subsubsection{RESISTOR DE 20M $\quad$ R}

Para a calibração da escala de $50 \mu \mathrm{A}$, utilizou-se como resistência de calibração, uma resistência de valor comercial cujo valor indicado no corpo, através de código de cores é igual a $20 \mathrm{M} \Omega$.

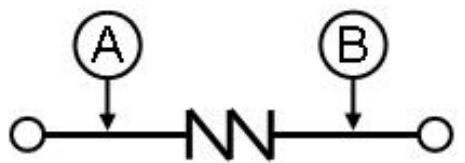

Fig. 4.13: Pontos de medição da Resistência de 20M $\Omega$

Durante o processo de medição do valor indicado pelo equipamento HP4156A, foi de:

\begin{tabular}{|c|c|}
\hline PONTOS & VALORES \\
\hline A e B & $20,529 \mathrm{M} \Omega$ \\
\hline
\end{tabular}

\subsubsection{RESISTOR DE $2 M \Omega$}

Para a calibração da escala de $500 \mu \mathrm{A}$, utilizou-se como resistência de calibração, uma resistência de valor comercial cujo valor indicado no corpo, através de código de cores é igual a $2 \mathrm{M} \Omega$. 


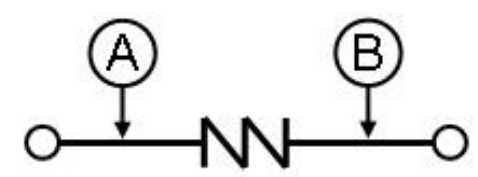

Fig. 4.14: Pontos de medição da Resistência de $2 \mathrm{M} \Omega$

Durante o processo de medição do valor indicado pelo equipamento HP4156A, foi de:

\begin{tabular}{|c|c|}
\hline PONTOS & VALORES \\
\hline A e B & $2,023 \mathrm{M} \Omega$ \\
\hline
\end{tabular}

\subsubsection{RESISTOR DE $200 \mathrm{k} \Omega$}

Para a calibração da escala de $5 \mathrm{~mA}$, utilizo-se como resistência de calibração, uma resistência de valor comercial cujo valor indicado no corpo, através de código de cores é igual a $200 \mathrm{k} \Omega$.

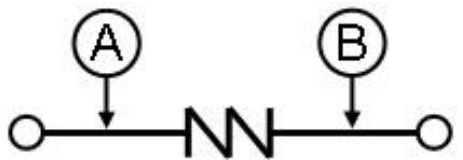

Fig. 4.15: Pontos de medição da Resistência de $200 \mathrm{k} \Omega$

Durante o processo de medição do valor indicado pelo equipamento HP4156A, foi de:

\begin{tabular}{|c|c|}
\hline PONTOS & VALORES \\
\hline A e B & $208,0451 \mathrm{k} \Omega$ \\
\hline
\end{tabular}

\subsubsection{GRÁFICOS DE CALIBRAÇÃO}

Foram realizados ensaios para avaliação do desempenho do SCEDEC desenvolvido, utilizando-se algumas amostras com micropontas fabricadas pela técnica HI-PS descrita anteriormente, num sistema de alto vácuo (em torno de $2 \times 10^{-6}$ Torr).

Com a fonte operando no modo "Controle Manual" foram tomadas medidas de Tensão x tempo (Figura 4.25a), Corrente $\mathrm{x}$ tempo (Figura 4.25b). Nesses testes procurou-se avaliar a estabilidade da medição. Também foram levantadas curvas VxI (Figura 4.23) e FowlerNordheim (Figura 4.24) para diferentes distâncias entre catodo (micropontas) e anodo. 
Através das figuras pode-se observar uma flutuação na Tensão de Saída da Fonte da ordem de $5 \%$ e uma flutuação de aproximadamente $0,1 \mu \mathrm{A}$.

\subsubsection{GRÁFICO V-I EM ESCALA DE CORRENTE DE 500nA.}

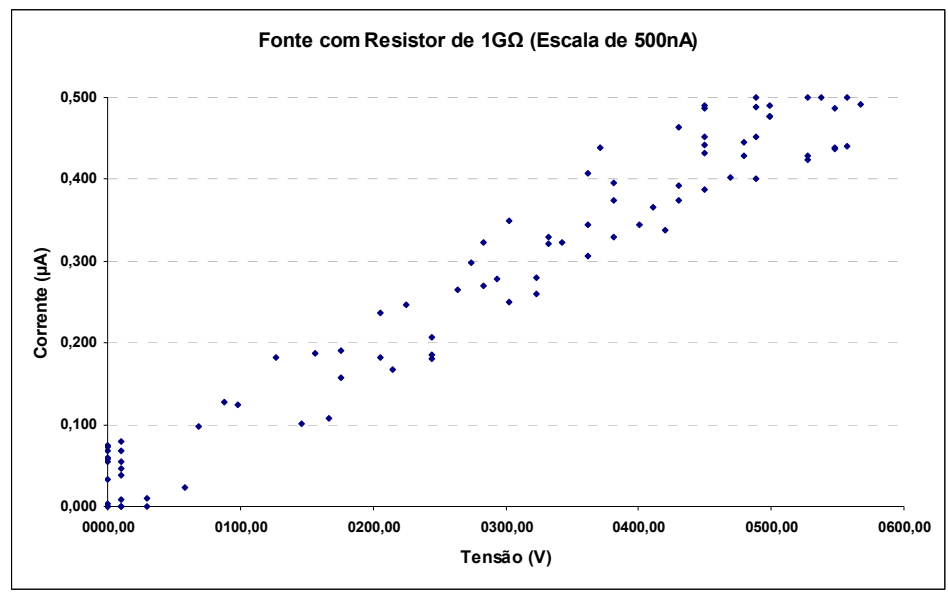

Fig. 4.16: Gráfico de Corrente x Tensão (500nA - resistor de 2G $\Omega$ )

\subsubsection{GRÁFICO V-I EM ESCALA DE CORRENTE DE 5 $\mu$ A.}

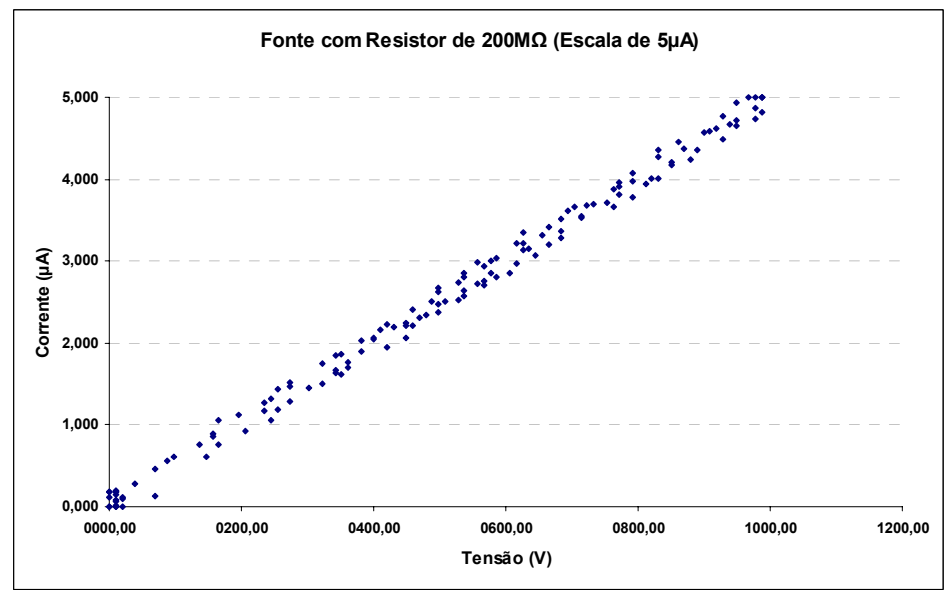

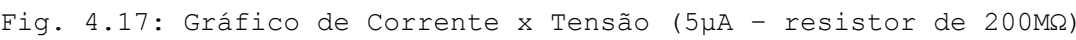


4.4.2.3. GRÁFICO V-I EM ESCALA DE CORRENTE DE 50 $\mu A$.

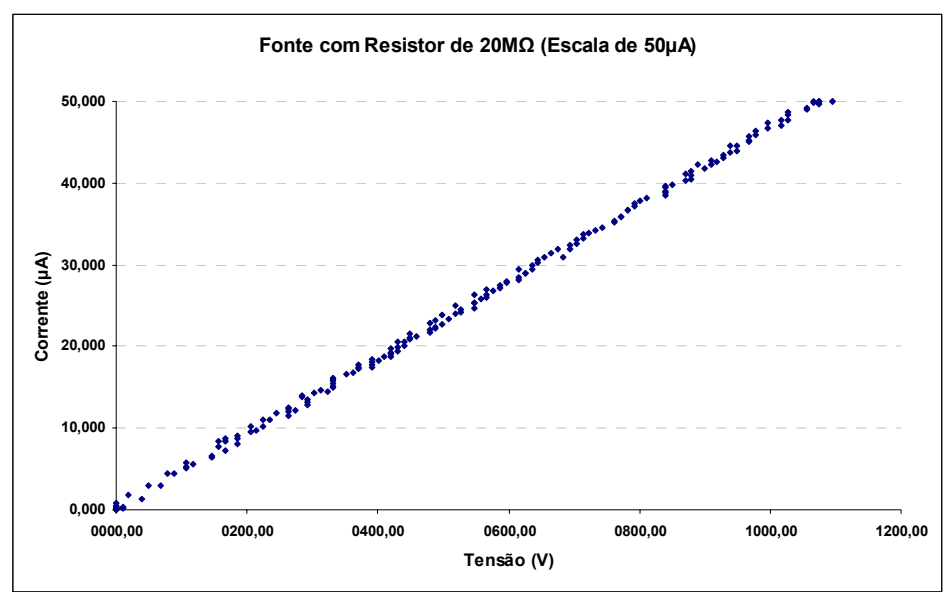

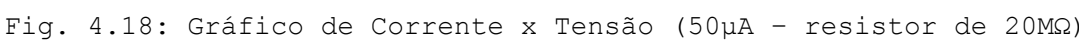

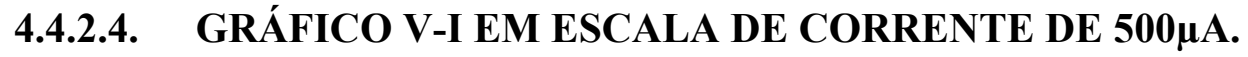

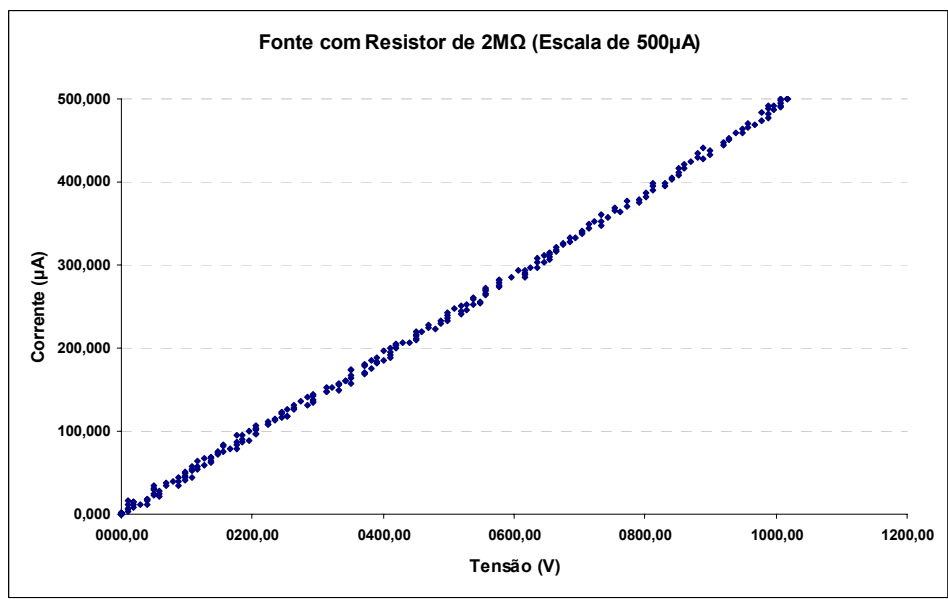

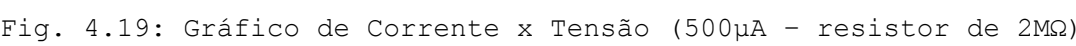

\subsubsection{GRÁFICO V-I EM ESCALA DE CORRENTE DE 5MA.}

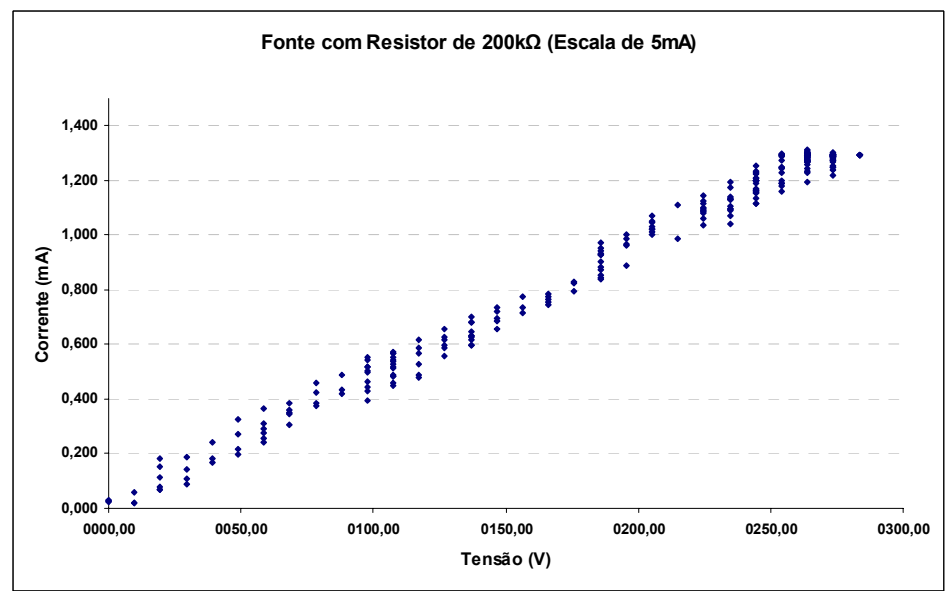

Fig. 4.20: Gráfico de Corrente x Tensão (5mA - resistor de $200 \mathrm{k} \Omega$ ) 
Como esperado, os gráficos V-I nos ensaios com resistores mostram uma rampa linear, Com escalas mais baixas, Figura 4.17, a dispersão dos pontos é visualmente mais evidente. Isso porque nas escala onde são indicados os valores de corrente elétrica de menor valor (500nA) a dispersão é da ordem de $\pm 9,87 \%$, enquanto na escala onde se indica os valores de maior corrente elétrica $(500 \mu \mathrm{A})$, por exemplo, os valores de dispersão das medições não passam de $\pm 0,93 \%$.

\subsubsection{GRÁFICOS OBTIDOS EM ENSAIOS COM DISPOSITIVOS FE}

Os gráficos apresentados na Figura 4.21 e 4.22, apresentam a curva V x I e o gráfico de Fowler-Nordheim para quatro distâncias distintas para a mesma amostra ensaiada, respectivamente. Os gráficos foram extraídos do sistema a partir de um ensaio real. Nesse ensaio, a distância entre as micropontas e o anodo foi modificada para avaliar o desempenho da emissão de campo. Nesses gráficos é possível observar a instabilidade da corrente de emissão dos dispositivos FE. Para resolver esse problema de instabilidade, os pesquisadores trabalham desenvolvendo novos materiais, novos métodos de fabricação ou tratamento na superfície das micropontas.

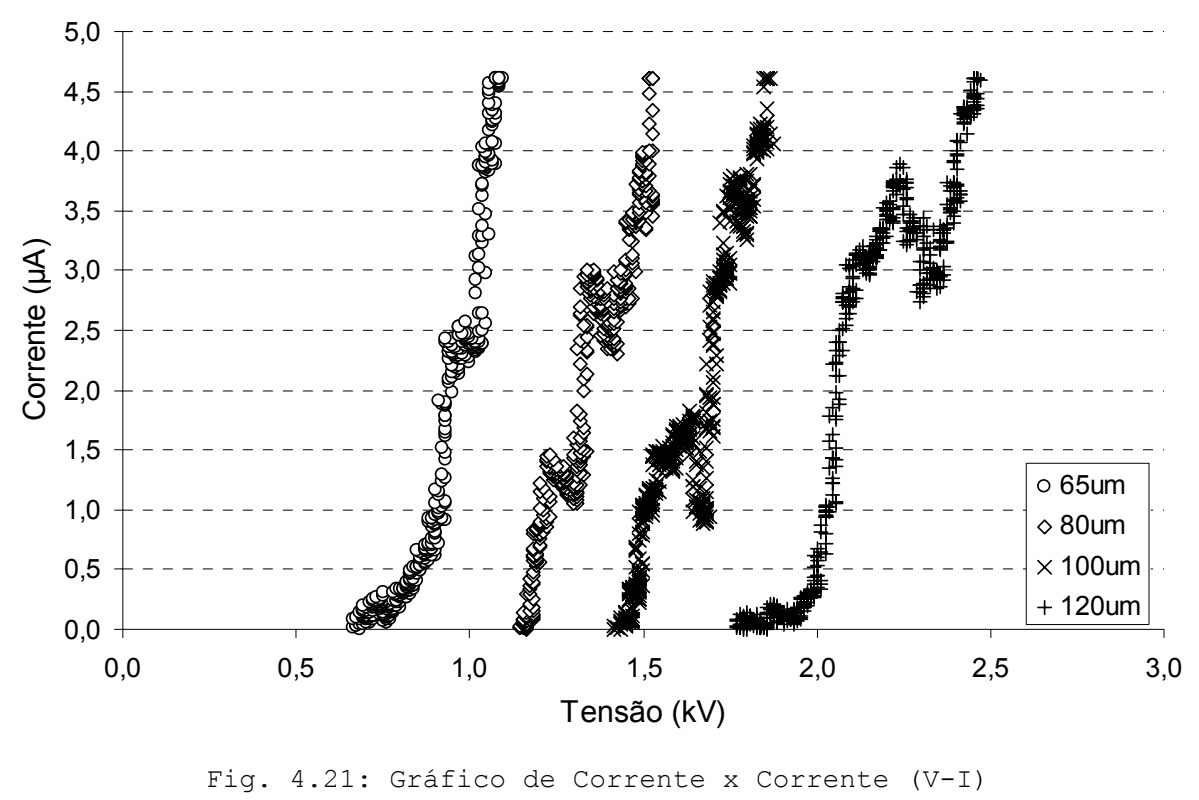




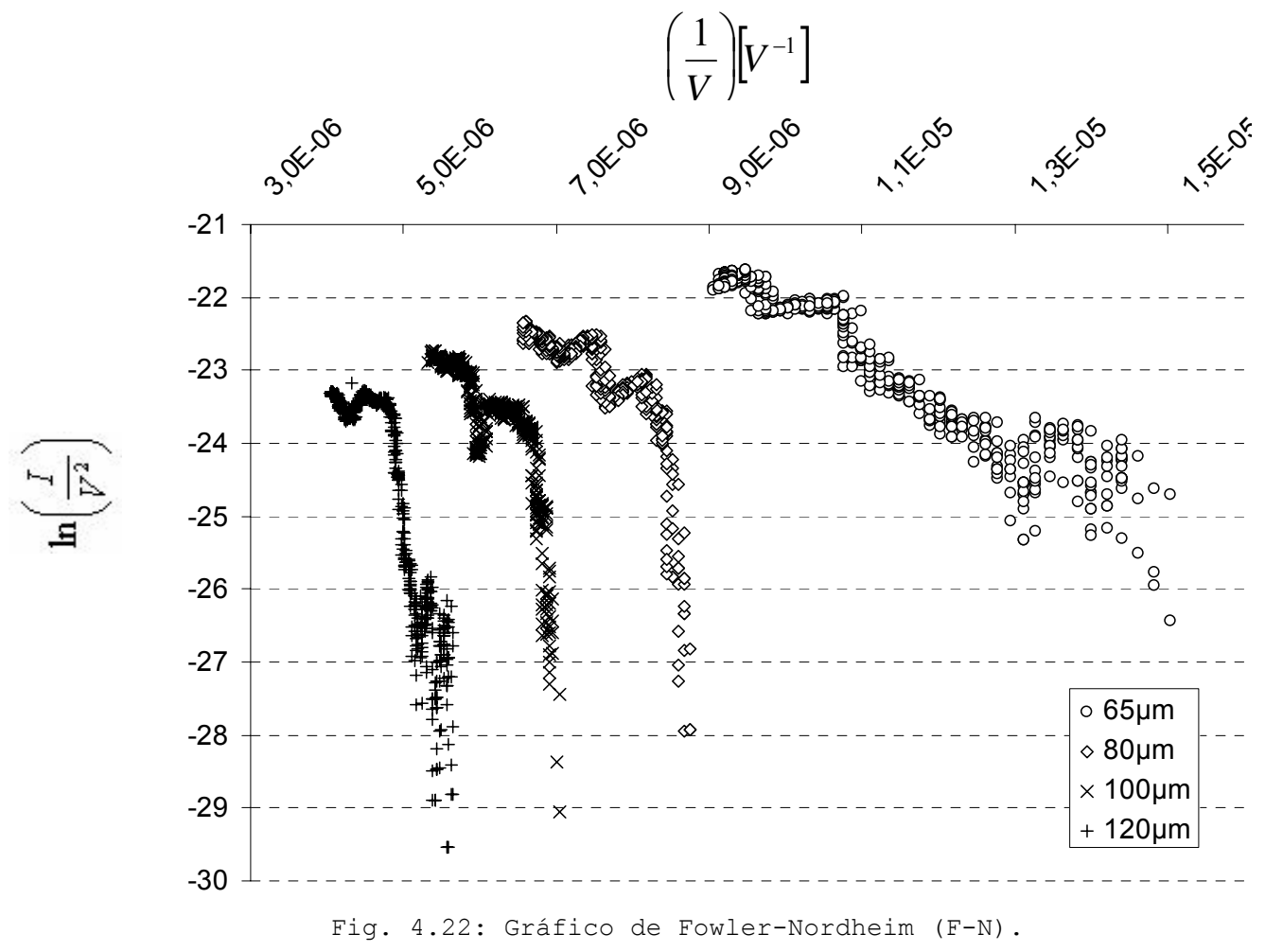

As Figuras 4.23 mostram dois exemplos de gráficos plotados "on-line" e com os dados posteriormente exportados. Eles foram extraídos de um dispositivo FE de silício não integrado, obtido com a técnica de HI-PS (DANTAS,2006). O primeiro, Figura 4.23a, mostra o curso da tensão de saída da fonte ao longo do tempo. O segundo, Figura 4.23b, mostra o curso da corrente ao longo do tempo.

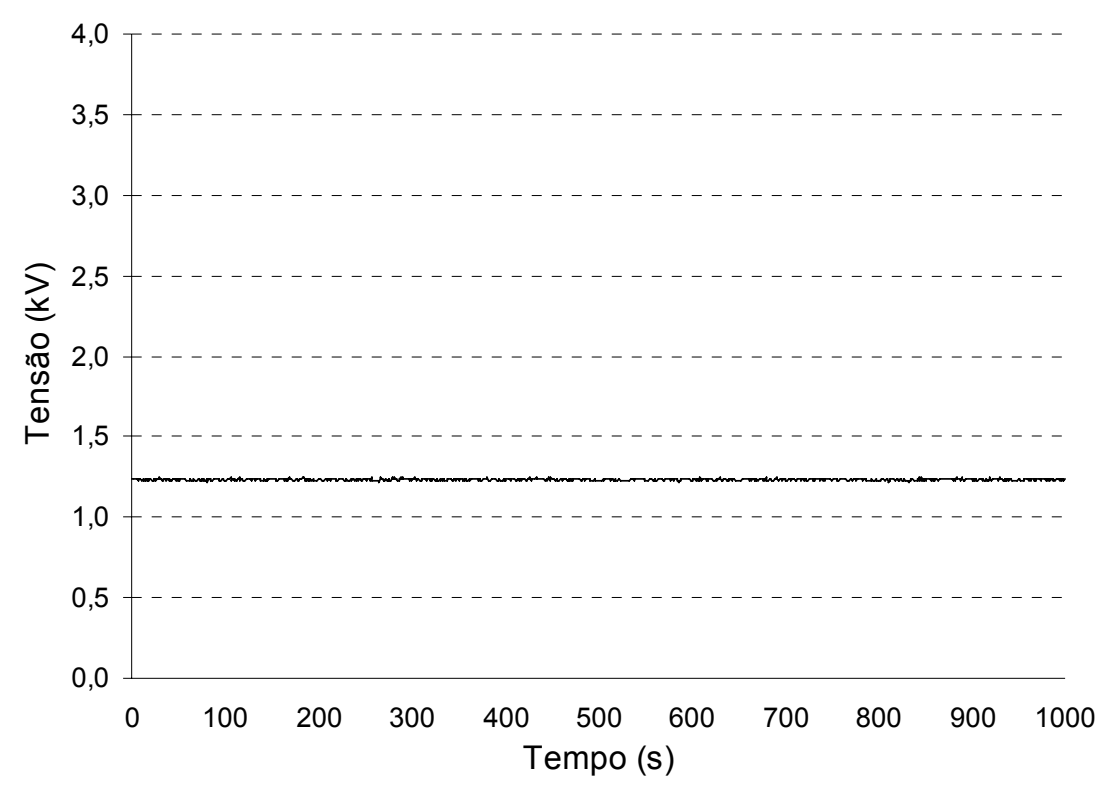

(a) 


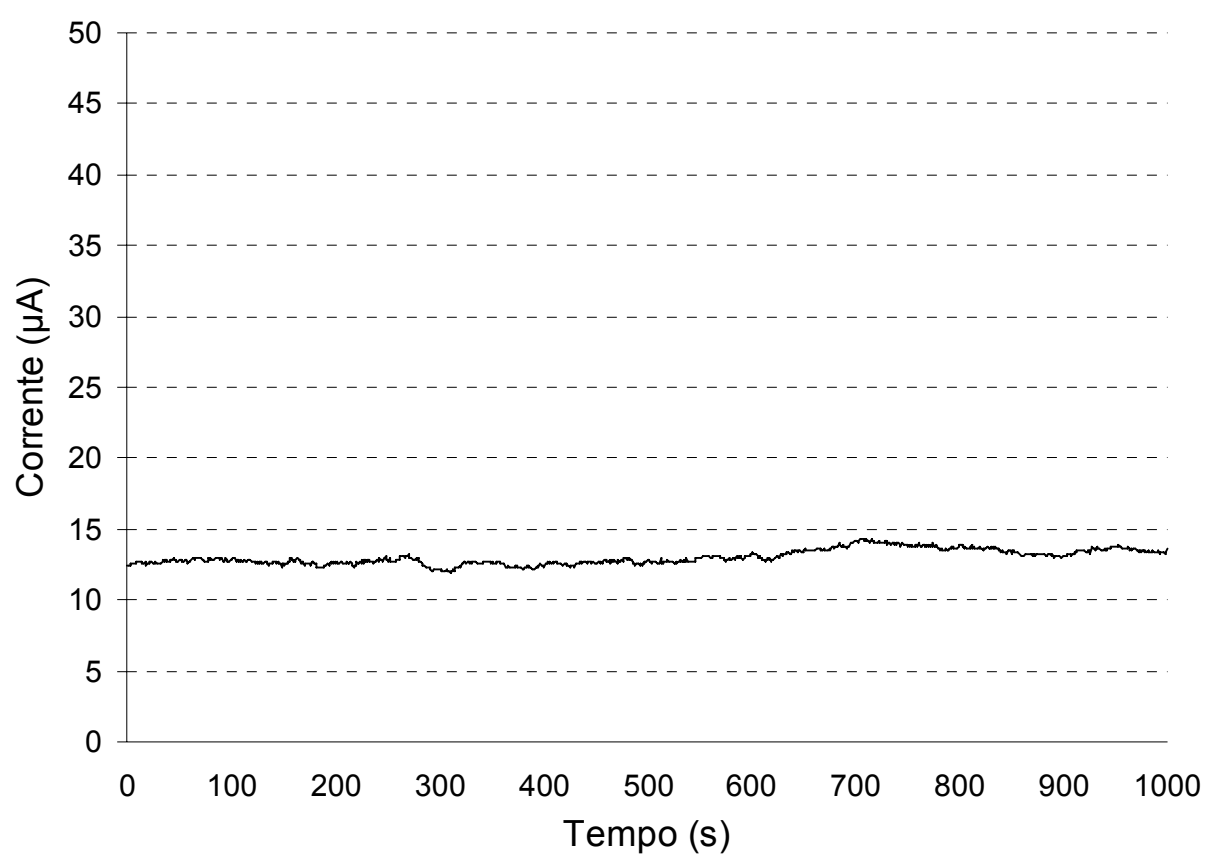

(b)

Fig. 4.23: Exemplo de um ensaio real

(a) Tensão e (b) Corrente versus tempo.

\subsection{COMPARAÇÃO DE INSTRUMENTOS VERSUS SCEDEC COM CARGA RESISTIVA}

A seguir são apresentados gráficos comparativos do desempenho das medições apresentadas no SCEDEC em comparação às medições indicadas nos instrumentos de calibração (HP34401A e Fluke8000A).

Nos gráficos é denominado de Fonte, a linha com as medições obtidas através do SCEDEC e é denominado de Instrumento, a linha com as medições obtidas com os instrumentos de calibração. 


\subsubsection{ESCALA DE 500nA}

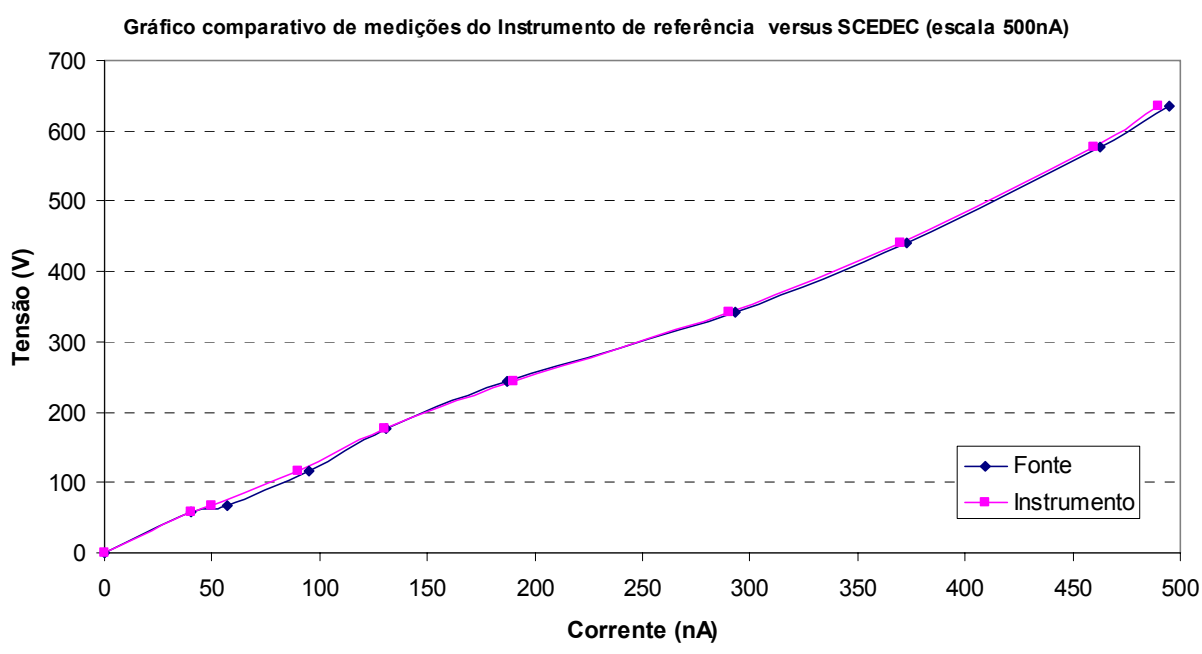

Fig. 4.24: Gráfico comparativo de Medições (escala de 500nA).

\subsubsection{ESCALA DE 5 $\mu \mathrm{A}$}

Gráfico comparativo de medições do Instrumento de referência versus SCEDEC (escala 5uA)

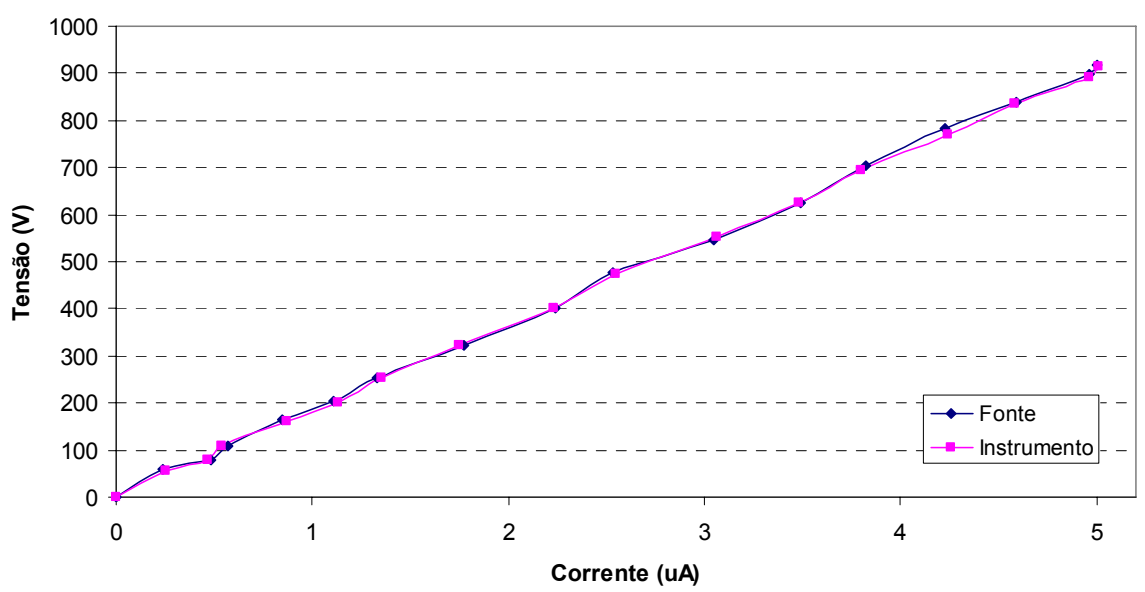

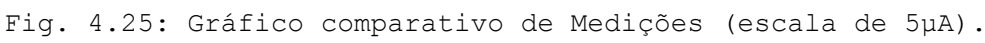




\subsubsection{ESCALA DE 50 $\mu$ A}

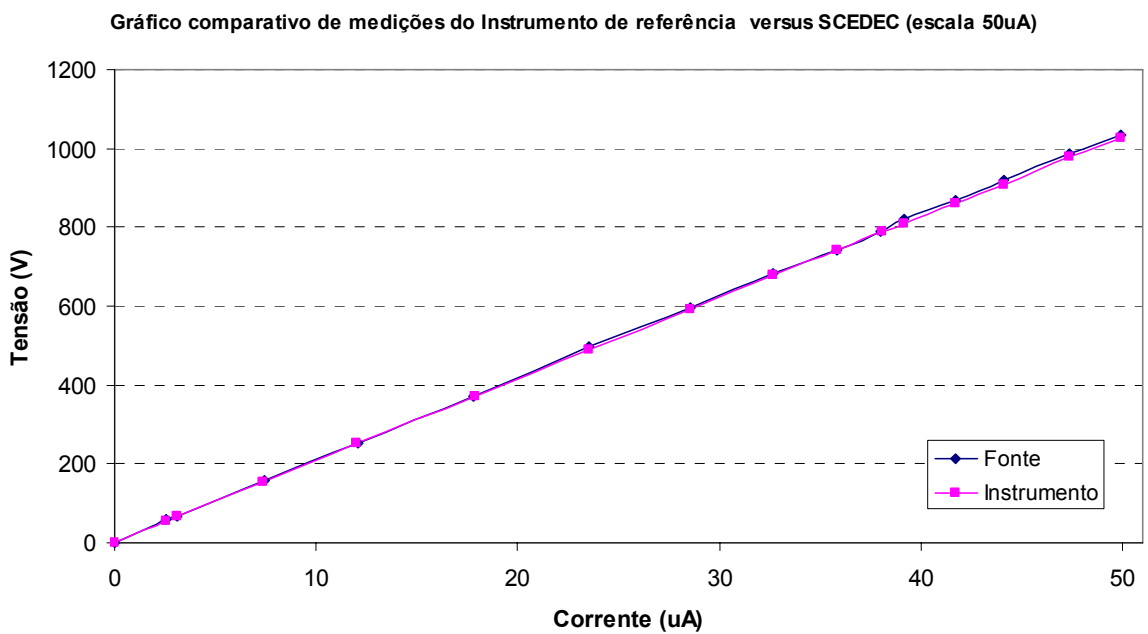

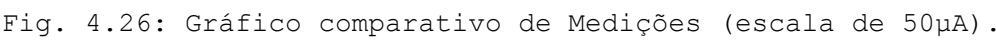

\subsubsection{ESCALA DE 500 $\mu \mathrm{A}$}

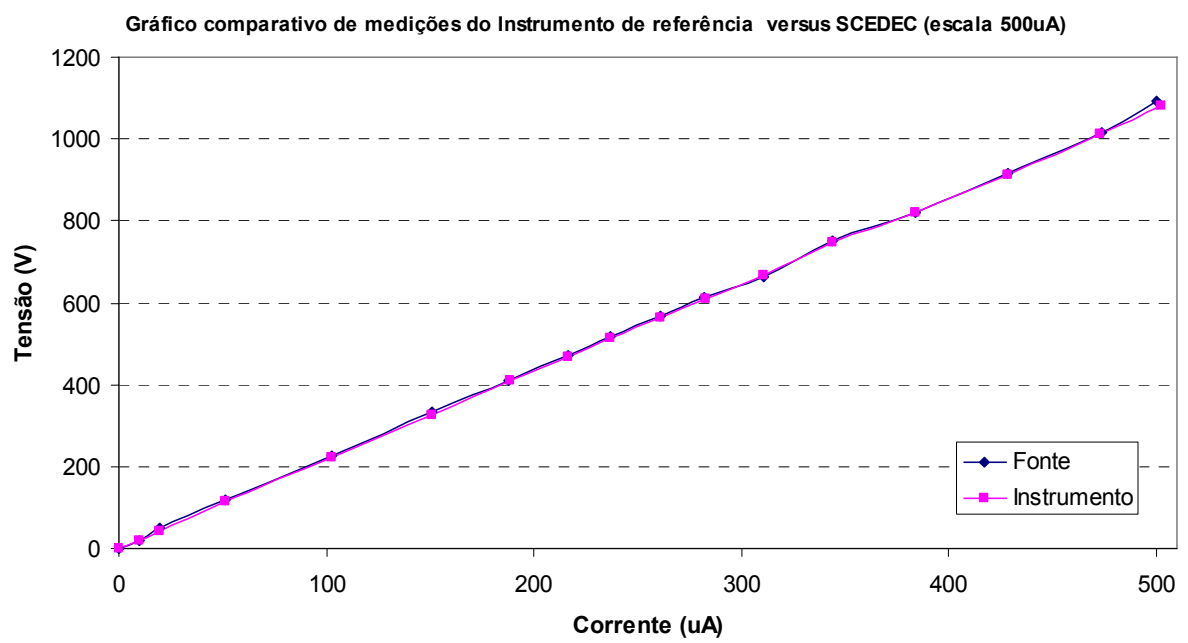

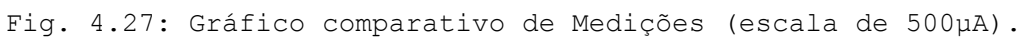




\subsubsection{ESCALA DE $5 \mathrm{~mA}$}

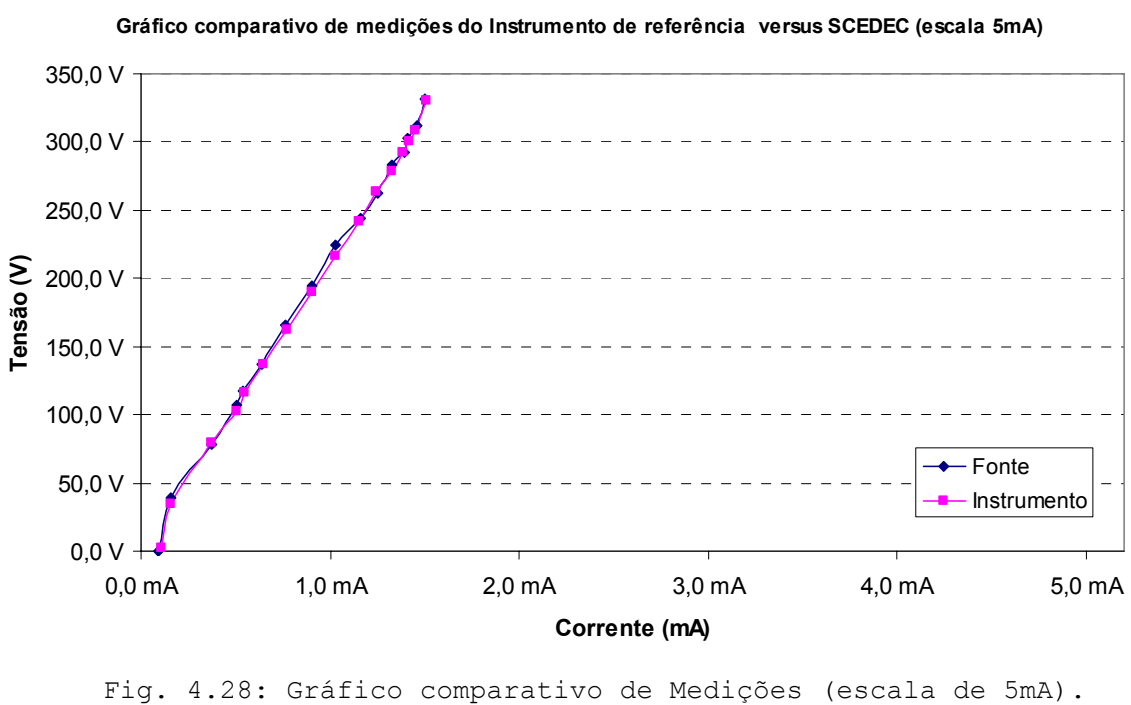

\subsection{COMPARAÇÃO DE INSTRUMENTOS VERSUS SCEDEC COM FED'S NA CÂMARA DE ALTO VÁCUO}

A seguir são apresentados gráficos comparativos do desempenho das medições apresentadas no SCEDEC em comparação às medições indicadas no instrumento fabricado pela empresa Keithley (Modelo 248 High Voltage Supply).

Esses ensaios foram feitos na câmara de ensaio de alto vácuo, utilizando-se uma amostra de FED de micropontas de silício identificada como amostra X58A (Fig 4.31) a uma distância de $50 \mu \mathrm{m}$ entre anodo e as micropontas, um degrau de incremento de tensão de $50 \mathrm{~V}$, numa faixa de 1000 à $3000 \mathrm{~V}$ e com pontos de amostragem a cada 10 segundos. Cada ensaio foi repetido três vezes com cada instrumento para que fosse possível verificar uma repetibilidade do comportamento do arranjo de ensaio.
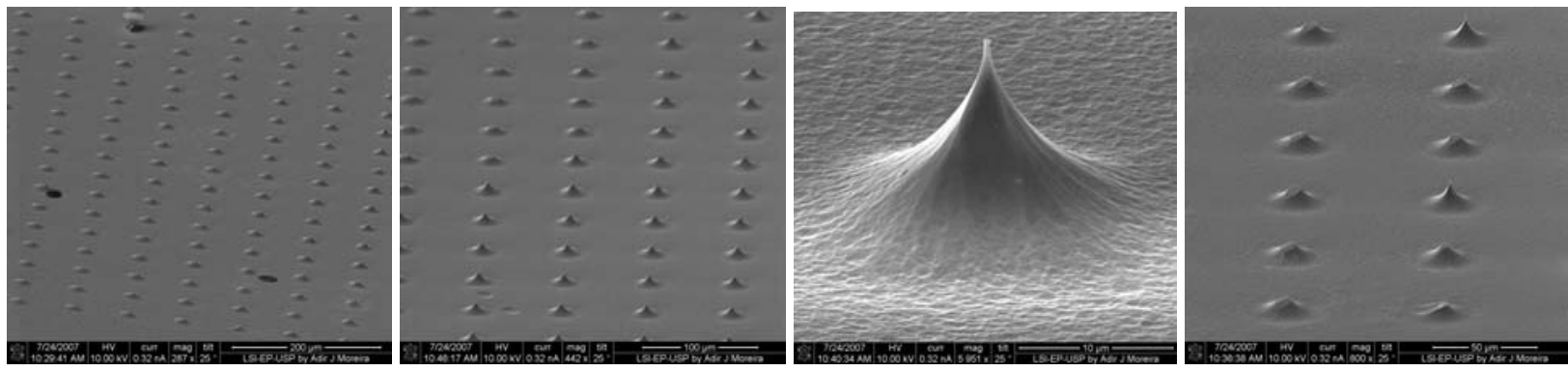

Fig. 4.29: Amostra X58A. 


\subsubsection{ENSAIO COM O VOLTIMETRO E AMPERÍMETRO KEITHLEY}

\section{Ensaios Keithley}

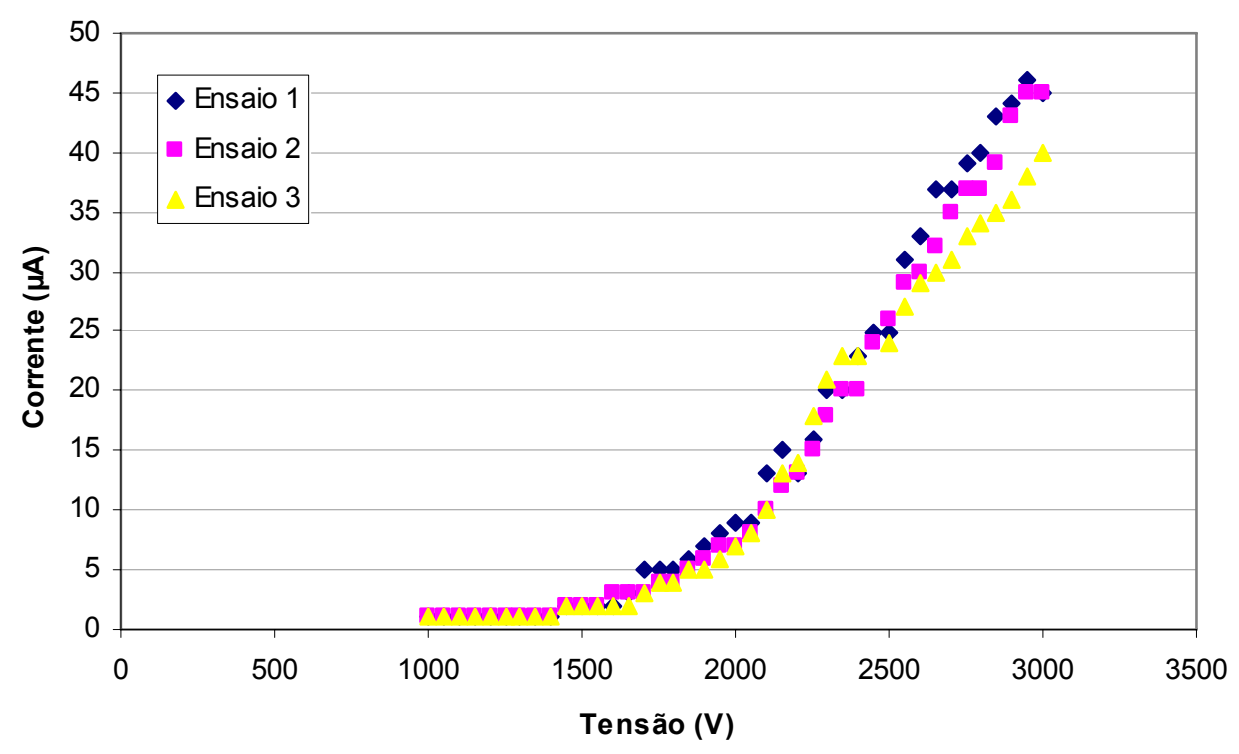

Fig. 4.30: Gráfico dos ensaios com o equipamento Keithley.

\subsubsection{ENSAIOS COM O SCEDEC}

Ensaios SCEDEC

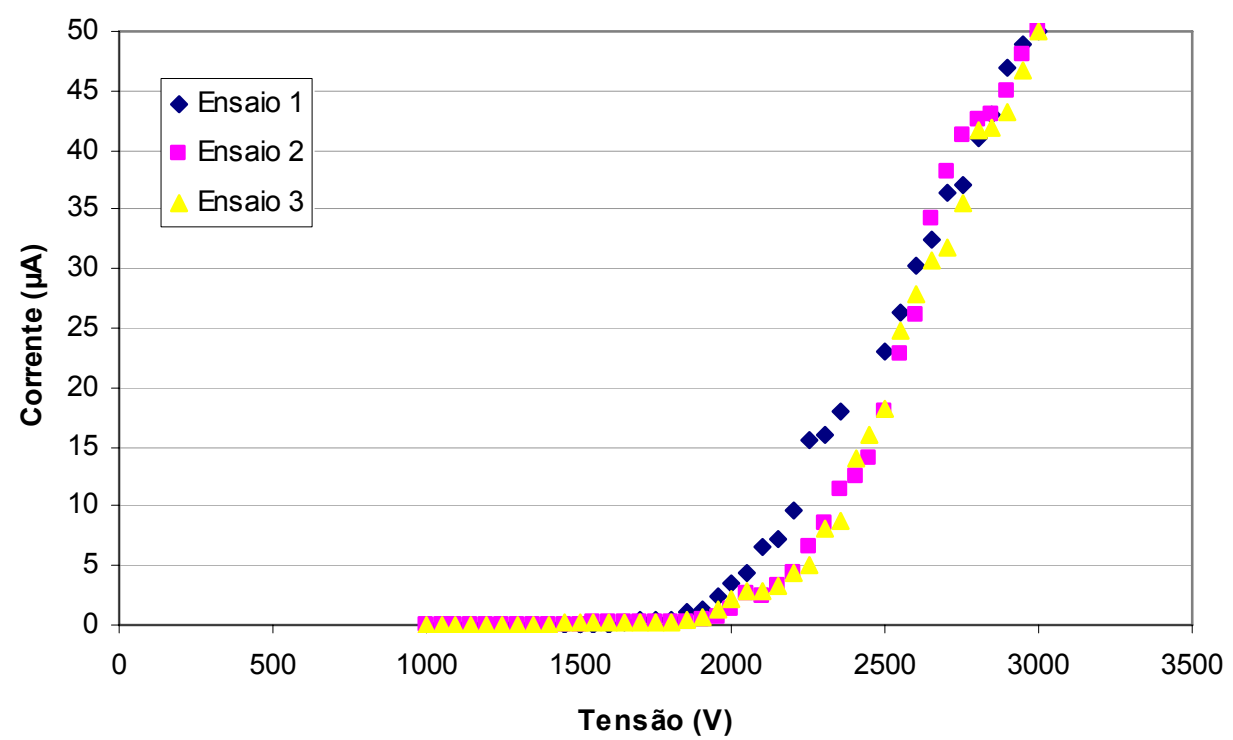

Fig. 4.31: Gráfico dos ensaios com o SCEDEC. 


\subsubsection{COMPARATIVO DAS MEDIÇÕES KEITHLEY VERSUS SCEDEC}

Comparação SCEDEC versus Keithley

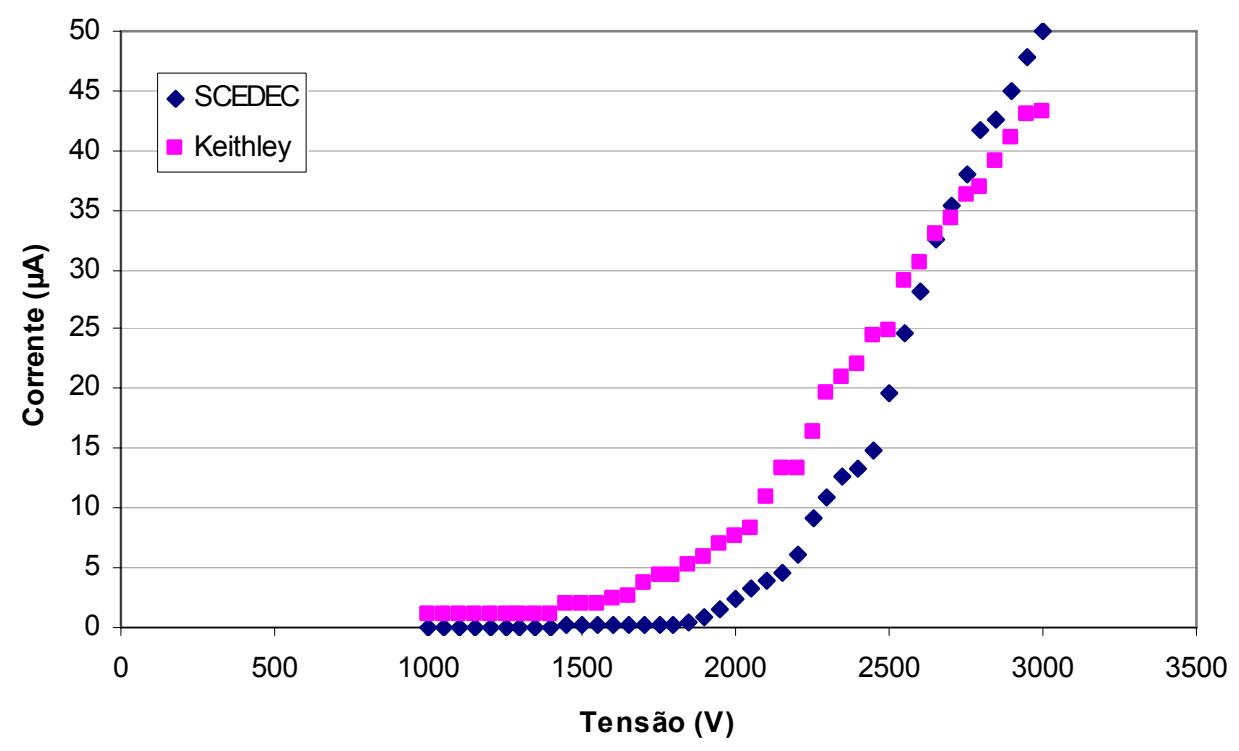

Fig. 4.32: Gráfico comparativo de Medições Keithley versus SCEDEC

Como é possível observar no gráfico acima, a curva obtida com os dois instrumentos de ensaio apresentam um comportamento exponencial e estão bem próximas, como esperado. Uma pequena diferença é observada no comportamento da curva, isso se deve ao fato de que o funcionamento dos dispositivos FED’s não é muito estável (XU,2000).

\subsection{CURVA DE CARGA DO SCEDEC}

A seguir é apresentada a curva de carga do SCEDEC.

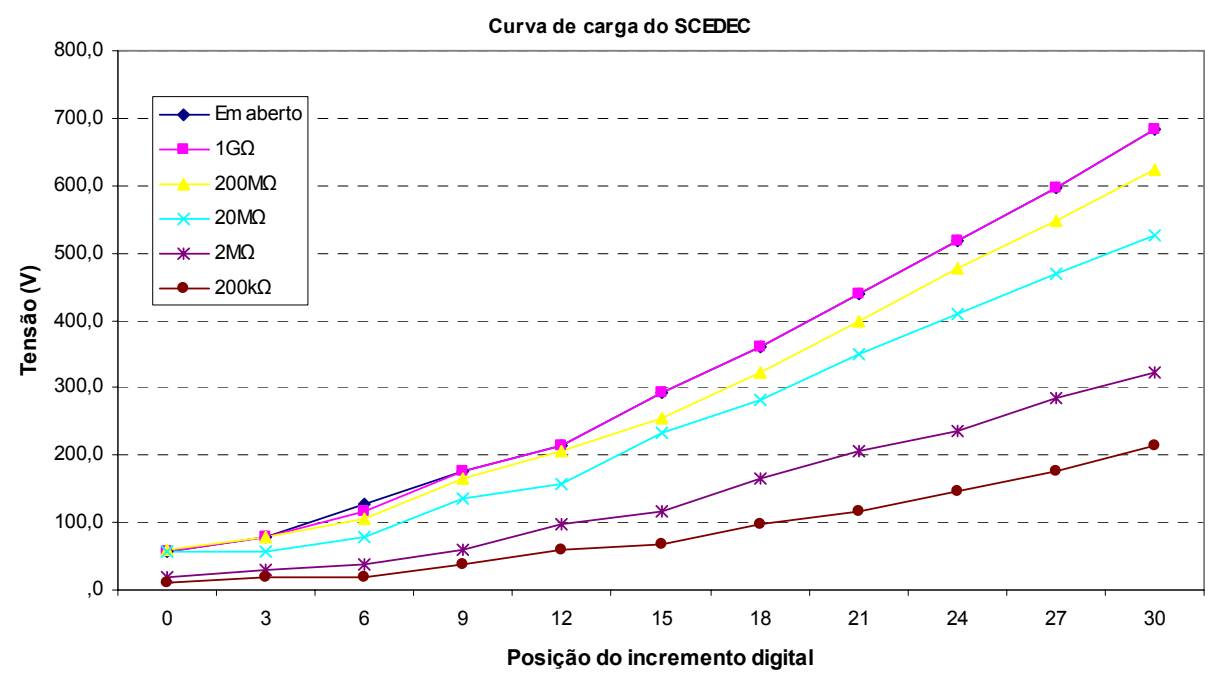

Fig. 4.33: Curva de carga do SCEDEC. 


\subsection{CURVA DE REGULAÇÃO DO SCEDEC}

A seguir é apresentada a curva de regulação do SCEDEC operando em modo de Controle Manual.

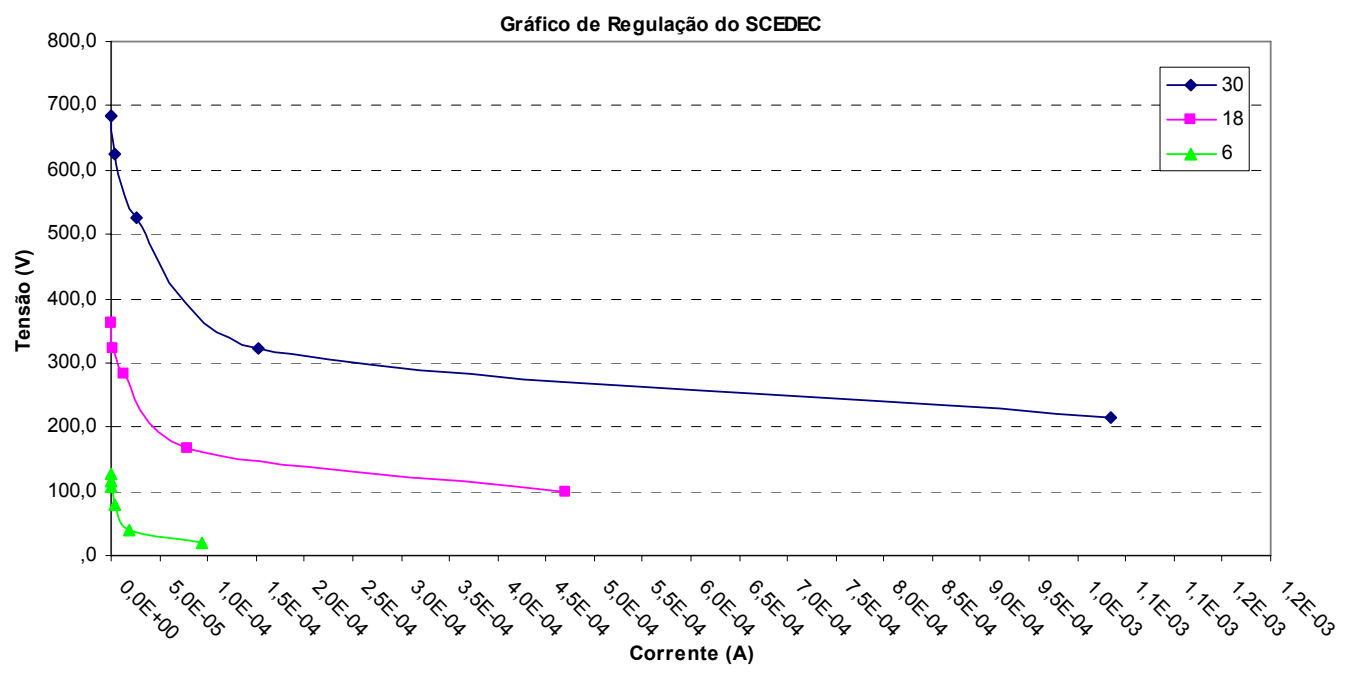

Fig. 4.34: Curva de regulação do SCEDEC. 


\section{CONCLUSÕES}

Concluímos que o SCEDEC se mostrou um Sistema que atende as expectativas concebidas durante a concepção do trabalho. Isso devido ao fato de se tratar de um Sistema desenvolvido especificamente e dedicado para essa aplicação e tendo os objetivos alcançados, principalmente por:

1) Se tratar de um sistema personalizado, com controle de Tensão Constante, Corrente Constante e Controle Manual;

2) Por disponibilizar a possibilidade de acompanhamento "on-line" dos ensaios através de cinco gráficos, Corrente x Tempo, Tensão x Tempo, Tensão x Corrente, Gráfico de FowlerNordheim e o Gráfico Duplo, onde simultaneamente são acompanhados os comportamentos da Tensão e da Corrente em função do Tempo;

3) Mostrar-se compatível com outros programas de análise, por possibilitar a exportação dos dados recolhidos durante os ensaios, com arquivo em extensão ".txt" e marcado com tabulações e caracteres seguindo o padrão ASCII;

4) Possibilitar a interação do usuário com os ensaios "on-line", isso porque os parâmetros dos ensaios podem ser alterados, mesmo no decorrer do ensaio;

5) Mostrar eficaz no que diz respeito à aplicação do conceito de "Instrumentação Virtual", pois além de todas as características já mencionadas anteriormente, o Sistema faz com que, através do programa HighVolt Controller, o usuário tenha todo o controle de acionamento da Fonte de Alta Tensão sem entrar em contato com a fonte. Inclusive, se quando se trata do acionamento e desacionamento da saída de Alta Tensão nos Bornes da Fonte;

6) O SCEDEC se mostrou também completamente autônomo em sua operação possibilitando que o usuário execute ensaios mesmo sem estar conectado com o computador, isso porque a Fonte de Alta Tensão possibilita a interação com os parâmetros do ensaio e acionamento da Alta Tensão nos bornes da Fonte remotamente, através das teclas localizadas no painel frontal.

7) Apresentar um bom comportamento no que diz respeito aos valores indicados pelo equipamento, pois como pôde ser observado nesta dissertação, em comparação com equipamentos comerciais de elevada precisão, o sistema apresentou valores precisos e completamente compatível com as necessidades dos ensaios, porém com a vantagem de ter sido desenvolvido de forma 
dedicada para aplicações acadêmicas e de forma personalizada, prevendo projeções futuras em sua utilização e com manuseio extremamente simples e intuitivo;

Como conclusão adicional, esse trabalho apresentou grande inovação e criatividade, pois com componentes relativamente simples e comerciais, além de aplicarmos conceitos básicos, podemos desenvolver uma solução que vem atendendo todas as expectativas e principalmente com um baixo custo. 


\section{PROJEÇÕES FUTURAS}

Como projeção futura, foi disponibilizada a possibilidade de implementação de atuação e controle de seis variáveis de uso geral, isso devido ao fato de que no pacote de transmissão, essas variáveis que foram configurados no programa HighVolt Controller, já estão incluídas. Outro fato que possibilita essa implementação é que no pacote de recebimento estão reservados as posições referentes aos valores medidos das variáveis de uso geral, com isso será possível o acompanhamento "on-line" do valor real medido.

Outra projeção está na possibilidade da implementação futura de um display gráfico na Fonte de Alta Tensão. Isso fará com que a autonomia aumente ainda mais, nos casos onde os ensaios estiverem sendo feitos sem a comunicação com o PC. Nele poderão ser implementados os gráficos on-line idênticos aos apresentados na tela do computador.

A implementação do armazenamento dos dados do ensaio na própria Fonte de Alta Tensão para futuramente ser descarregado num PC, aumentaria a sua portabilidade, além da implementação de uma comunicação USB no lugar da RS-232, poderia aumentar a velocidade com que os dados podem ser recolhidos e aumentar a velocidade com que os dados podem ser transmitidos, isso porque a capacidade de transmissão desse tipo de porta de comunicação em comparação com a RS232 é muito maior. Essa implementação relaciona-se também à reestruturação da isolação ótica dos dados transmitidos, pois os fotoacopladores devem acompanhar essa velocidade maior de comutação. 


\section{BIBLIOGRAFIA}

AGUIRRE IÑIGO J. OLEAGORDIA, RAFAEL URRETABIZKAYA GARBUS, RICARDO CHAPARRO SÁNCHEZ; "Signal Generation based on Low Cost Virtual Instrumentation", Proceedings of the Electronics, Robotics and Automotive Mechanics Conference (CERMA'06) IEEE, (2006);

BADI, N.; HE, K.; NAIR, A.; BENSAOULA, A.; "Design and fabrication of field emissionbased pressure microsensors". 17th International Vacuum Nanoelectronics Conference (IVNC), p. $24-25$ (2004);

BALE, M.; PALMER, R.E.; "Microfabrication of silicon tip structures for multiple-probe scanning tunneling microscopy”. Journal of Vacuum Science Technology B, v. 20, n.1, p. 364369 (2002);

BECK, THOMAS; "Current trends in the design of automotive electronic systems Design", Automation and Test Conference, (2001);

D.S.BENITEZ, A.ZAIDI, A.FITCHET, P.A.GAYDECKI AND A.P.FITZPATRICK; "Virtual instrumentation for clinical assessment of cardiovascular and autonomic function". IEE Proc.Sci. Meus. Technol., Vol. 147, No. 6, November - MEDICAL SIGNAL PROCESSING, p. $397-$ 402 (2000);

BORGES ADRIANA PAULA, "Instrumentação Virtual Aplicada a Um Laboratório com Acesso pela Internet", Trabalho de Mestrado, (2002);

BURGESS, R.E.; KROEMER, H.; HOUSTON, J.M.; "Corrected values of Fowler - Nordheim field emission functions $v(y)$ and $s(y) "$. Physical Review, v. 90, n. 4, p. 515 (1953);

CHUNG, C.H.; SONG, Y.H.; HWANG, C.S.; AHN, S.D.; KIM, B.C.; CHO, Y.R.; LEE, J.H.; CHO, K.I.; "Effects of surface treatment of field emitter arrays on electrical characteristics of active matrix cathode". Thin Solid Films, n. 460, p. 201-205 (2004);

CRISTALDI, LOREDANA; FERRERO, ALESSANDRO; PIURI, VINCENZO; "Programable Instruments, Virtual Instruments, and Distributed Measurement Systems: What is realy useful, innovative and Technically Sound?" IEEE Instrumentation \& Measurement Magazine, September, (1999);

* De acordo com

ASSOCIAÇÃO BRASILEIRA DE NORMAS TÉCNICAS. NBR 6023: informação de documentação: referência: elaboração. Rio de Janeiro, 2002. 
DANTAS, M.O.S.; GALEAZZO, E.; PERES, H.E.M.; FERNANDEZ ，F.J.R.; "HI-PS Technology Applied on Si Tips Fabrication for Field Emission Devices”, Ibersensor-2006, (2006);

DANTAS, M.O.S.; "Desenvolvimento de Matrizes de Emissores de Elétrons por Efeito de Campo pela Técnica HI-PS“. Tese de Doutorado - em andamento (2006);

DEBSKI, T.; BARTH, W.; RANGELOW, I.W.; BIEHL, S.; GRABIEC, P.; BEKH, I.I.; LUSHKIN, A.E.; IL'CHENKO, L.G.; IL'CHENKO, V.V.; KOSTIC, I.; HUDEK, P.; MITURA, S.; "Gated field emitter arrays". Microelectronic Engineering, n. 57-58, p. 813-818 (2001);

DOLAN, W.W.; "Current density tables for field emission theory". Physical Review, v. 91, n. 3, p. 510-511 (1953);

HALIT EREN AND TITUS RASAN; “A Virtual Instrumentation for Monitoring Induction Machines", 0-7803-4879-6/98 IEEE, p. 141-146, (1998);

FUNG, Y.M.; CHEUNG, W.Y.; WILSON, I.H.; CHEN, D.; XU, J.B.; WONG, S.P.; KWOK, R.W.M.; "Electron field emission characteristics of textured silicon surface". Journal of Vacuum Science Technology B, v. 19, n.3, p. 884-887 (2001);

FURSEY, G.N.; "Field emission in vacuum micro-electronics". Applied Surface Science, n. 215, p. 113-134 (2003);

FURSEY, G.N.; "Field emission in vacuum microelectronics". Ed. Kluwer Academic / Plenum Publisher, New York, USA (2005);

GALEAZZO, E.; SALCEDO, W.J.; PERES, H.E.M.; FERNANDEZ, F.J.R.; "Porous silicon patterned by hydrogen ion implantation". Sensors and Actuators B, p. 343-346 (2001);

GEORGE BOHY, V.JAYASHANKAR, V. JAGADEESH KUMAR; "Virtual Instrument Based Instrumentation for Harmonic Current Emission Measurement", Proceedings of INCEMIC, p. 399-402 (2003);

GETREU, IAN; “Modeling the Bipolar Transistor" Tektronix, Inc. (1978);

GHODSIAN, B.; PARAMESWARAN, M.; SYRZYCKI, M.; "Gas detector with low-cost micromachined field ionization tips". IEEE Electron Device Letters, v. 19, n. 7, p. 241-243 (1998);

GOLDBERG, HAROLD; "What is Virtual Instrumentation" IEEE Instrumentation \& Measurement Magazine, December, (2000); 
GÖLZHÄUSER, A.; "Nanolithography and electron holography with ultrasharp field emitters". Applied Surface Science, n. 141, p. 264-273 (1999);

GRISS, P.; ENOKSSON, P.; LAAKSO, H.K.T.; MERILÄINEN, P.; OLLMAR, S.; STEMME, G.; "Micromachined electrodes for biopotential measurements". Journal of Microelectromechanical Systems, v. 10, n. 1, p. 10-16 (2001);

HEUSCHKEL, M. O.; FEJTL, M.; RAGGENBASS, M.; BERTRAND, D.; RENAUD, P.; "A three-dimensional multi-electrode array for multi-site stimulation and recording in acute brain slices". Journal of Neuroscience Methods, p. 135-148 (2002);

HIRAYAMA, S.; WATANABE, F.; TAKAHASHI, T.; MOTOOKA, T.; "Field emission current from Si tip: ultra-fast time resolved measurements". Surface Science, n.515, p. 369-376 (2002);

HUQ, S.E.; PREWETT, P.D.; SHE, J.C.; DENG, S.Z.; XU, N.S.; "Field emission from amorphous diamond coated silicon tips”. Materials Science and Engineering B, n. 74, p. 184187 (2000);

JÄNCHEN, G.; HOFFMANN, P.; KRIELE, A.; LORENZ, H.; KULIK, A.J.; DIETLER, G.; "Mechanical properties of high-aspect-ratio atomic-force microscope tips". Applied Physics Letters, v. 80, n. 24, p. 4623-4625 (2002);

JENKINS, D.W.; "Emission area of a field emitter array". IEEE Transactions On Electron Devices, v. 40, n. 3, p. 666-672 (1993);

JENSEN, K.L.; LAU, Y.Y.; MCGREGOR, D.; "Photon assisted field emission from a silicon emitter". Solid-State Electronics, n. 45, p. 831-840 (2001);

JENSEN, K. L.; “Theory and simulation of field emission from microstructures". Naval Research Laboratory: Cathode Workshop, Washington DC, CD-ROM (2001);

KLEPS, I.; ANGELESCU, A.; SAMFIRESCU, N.; GIL, A.; CORREIA, A.; "Study of porous silicon, silicon carbide and DLC coated field emitters for pressure sensor application". SolidState Electronics, n. 45, p. 997-1001 (2001);

LEE, Y.H.; LEE, S.J.; JU, B.K.; OH, M.H.; "Light-emitting devices using micromachined Si-tip mirror arrays". Sensors and Actuators, n. 80 p. 138-142 (2000);

LI, X.; YANG, C.; BAI, G.; ZHANG, F.; LIAO, F.; FENG, J.; DING, M.; DU, Y.; "Investigation of FEAs applied in vacuum electron gun”. Applied Surface Science, n. 215, p. 249-252 (2003); 
LI WEIBO, CHENGXIONG MAO, AND JIMING LU; "Study of the Virtual Instrumentation Applied to Measure Pulsed Heavy Currents", IEEE Transactions on Instrumentation and Measurement, Vol. 54, No. 1, February, p. 284-288, (2005);

MADOU, M.; "Fundamentals of microfabrication". Editora CRC, p. 145-208 (1997);

MAMMANA, V.P.; "Novos processos e configurações para mostradores planos de informação". Tese de Doutorado apresentada ao Instituto de Física da Universidade de São Paulo (2000);

MARINO, P.; NOGUEIRA, J.; HERNANDEZ, H.; "Laboratory of virtual instrumentation for industrial electronics"; Proceedings of IEEE International Conference on Industrial Technology, Volume: 2, (2000);

MITA, M.; KAWARA, H.; TOSHIYOSHI, H.; ENDO, J.; FUJITA, H.; "Bulk Micromachined Tunneling Tips Integrated With Positioning Actuators". Journal of Microelectromechanical Systems, v. 14, n. 1, p. 23-28 (2005);

RESNIK, D.; VRTACNIK, D.; ALJANCIC, U.; MOZEK, M.; AMON, S.; "Different aspect ratio pyramidal tips obtained by wet etching of (100) and (111) silicon". Microelectronics Journal, v. 34, p. 591-593 (2003);

SALANC, E.; HAMMADI, Z.; MORIN, R.; "A new approach to gas field ion sources". Ultramicroscopy, n. 95, p. 183-188 (2003);

SILVA, S.R.P.; CAREY, J.D.; CHEN, G.Y.; COX, D.C.; FORREST, R.D.; POA, C.H.P.; SMITH, R.C.; TANG, Y.F.; SHANNON, J.M.; "Nanoengineering of materials for field emission display technologies". IEE Proc.-Circuits Devices Syst., v. 151, n. 5, p. 489-496 (2004);

PAVOL SPANIK, LIBOR HARGAS, MIROSLAV HRIANKA, IVAN KOZEHUBA; “Application of Virtual Instrumentation LabVIEW for Power Electronic System Analysis”, 14244-0121-6/06 IEEE, p. 1699-1702 , (2006);

SPOELDER, HANS J. W; "Virtual Instrumentation and Virtual Environments"; IEEE Instrumentation \& Measurement Magazine, September, (1999);

SUN, C.; "Microengineered sensor devices with field emission electron sources". Dissertação apresentada ao New Jersey Institute of Technology, p. 1-124 (1998);

TALIN, A.A.; DEAN, K.A.; JASKIE, J.E.; "Field emission displays: a critical review". SolidState Electronics, n. 45, p. 963-976 (2001);

TANER, A. H.; BRIGNELL, J. E.; "Virtual Instrumentation and Intelligent Sensors", Sensors and Actuators A, Vol. 61, p. 427-430, (1997); 
THIEBAUD, P.; BEURET, C.; ROOIJ, N.F.; KOUDELKA-HEP, M.; "Microfabrication of Pttip microelectrodes". Sensors and Actuators B, p. 51-56 (2000);

CHANGTING WANG, AND ROBERT X. GAO; "A Virtual Instrumentation System for Integrated Bearing Condition Monitoring", IEEE Transactions on Instrumentation and measurement, Vol. 49, No. 2, APRIL, p. 325-332 (2000);

WISITSORAT, A.; “Micropatterned diamond vacuum field emission devices”. Dissertação apresentada à Vanderbilt University, Nashville Tennessee (2002);

XU, N.S.; HUQ, S.E.; "Novel cold cathode materials and applications”. Materials Science and Engineering, n. 48, p. 47-189, (2005);

ZHU, W.; "Vacuum micro-electronics". Ed. Wiley Inter-Science, New York, USA, (2001);

ZUUK, A.; HEERKENS, C.T.H.; VEEN, A.H.V.; TEEPEN, T.F.; WIELAND, M.J.; GROENING, O.; KRUIT, P.; "Fabrication and characterization of silicon carbide field-emitter array". Microelectronic Engineering, n. 73-74, p. 106-110 (2004); 


\section{ANEXOS}

\subsection{PIC18F452}

\section{Nin \\ MICROCHIP}

\section{8/40-pin High Performance, Enhanced FLASH Microcontrollers with 10-Bit A/D}

High Performance RISC CPU:

- C compiler optimized architecture/instruction set - Source code compatible with the PIC16 and PIC17 instruction sets

- Linear program memory addressing to 32 Kbytes - Linear data memory addressing to 1.5 Kbytes

\begin{tabular}{|c|c|c|c|c|}
\hline \multirow{2}{*}{ Device } & \multicolumn{2}{|c|}{$\begin{array}{c}\text { On-Chip Program } \\
\text { Memory }\end{array}$} & \multirow{2}{*}{$\begin{array}{c}\text { On-Chip } \\
\text { RAM } \\
\text { (bytes) }\end{array}$} & \multirow{2}{*}{$\begin{array}{c}\text { Data } \\
\text { EEPROM } \\
\text { (bytes) }\end{array}$} \\
\hline & $\begin{array}{l}\text { FLASH } \\
\text { (bytes) }\end{array}$ & $\begin{array}{l}\text { \# Single Word } \\
\text { Instructions }\end{array}$ & & \\
\hline PIC18F242 & $16 \mathrm{~K}$ & 8192 & 768 & 256 \\
\hline PIC18F252 & $32 \mathrm{~K}$ & 16384 & 1536 & 256 \\
\hline PIC18F442 & $16 \mathrm{~K}$ & 8192 & 768 & 256 \\
\hline IC18F452 & $32 \mathrm{~K}$ & 16384 & 1536 & 256 \\
\hline
\end{tabular}

- Up to 10 MIPs operation:

- DC - $40 \mathrm{MHz}$ osc./clock input

- $4 \mathrm{MHz}-10 \mathrm{MHz}$ osc./clock input with PLL active

- 16-bit wide instructions, 8-bit wide data path

- Priority levels for interrupts

- 8 × 8 Single Cycle Hardware Multiplier

\section{Peripheral Features:}

- High current sink/source $25 \mathrm{~mA} / 25 \mathrm{~mA}$

- Three external interrupt pins

- Timer0 module: 8-bit/16-bit timer/counter with 8-bit programmable prescaler

- Timer1 module: 16 -bit timer/counter

- Timer2 module: 8-bit timer/counter with 8-bit period register (time-base for PWM)

- Timer3 module: 16 -bit timer/counter

- Secondary oscillator clock option - Timer1/Timer3

- Two Capture/Compare/PWM (CCP) modules. CCP pins that can be configured as:

- Capture input: capture is 16-bit, max. resolution $6.25 \mathrm{~ns}$ (Tcr/16)

- Compare is 16-bit, max. resolution $100 \mathrm{~ns}$ (TCY)

- PWM output: PWM resolution is 1- to 10-bit, max.PWM freq. @: 8-bit resolution =156 kHz 10-bit resolution $=39 \mathrm{kHz}$

- Master Synchronous Serial Port (MSSP) module, Two modes of operation:

- 3-wire SPITM (supports all 4 SPI modes)

- $\mathrm{I}^{2} \mathrm{C}^{\mathrm{TM} M}$ Master and Slave mode
Peripheral Features (Continued):

- Addressable USART module:

- Supports RS-485 and RS-232

- Parallel Slave Port (PSP) module

\section{Analog Features:}

- Compatible 10-bit Analog-to-Digital Converter module (A/D) with

- Fast sampling rate

- Conversion available during SLEEP

- Linearity $\leq 1$ LSb

- Programmable Low Voltage Detection (PLVD)

- Supports interrupt on-Low Voltage Detection

- Programmable Brown-out Reset (BOR)

\section{Special Microcontroller Features:}

- 100,000 erase/write cycle Enhanced FLASH program memory typical

- $1,000,000$ erase/write cycle Data EEPROM memory

- FLASH/Data EEPROM Retention: > 40 years

- Self-reprogrammable under software control

- Power-on Reset (POR), Power-up Timer (PWRT) and Oscillator Start-up Timer (OST)

- Watchdog Timer (WDT) with its own On-Chip RC Oscillator for reliable operation

- Programmable code protection

- Power saving SLEEP mode

- Selectable oscillator options including

- 4X Phase Lock Loop (of primary oscillator)

- Secondary Oscillator $(32 \mathrm{kHz})$ clock input

- Single supply $5 \mathrm{~V}$ In-Circuit Serial Programming TM (ICSP ${ }^{\mathrm{M} M}$ ) via two pins

- In-Circuit Debug (ICD) via two pins

\section{CMOS Technology:}

- Low power, high speed FLASH/EEPROM technology

- Fully static design

- Wide operating voltage range ( $2.0 \mathrm{~V}$ to $5.5 \mathrm{~V})$

- Industrial and Extended temperature ranges

- Low power consumption:

- < 1.6 mA typical @ 5V, 4 MHz

- $25 \mu \mathrm{A}$ typical @ 3V, 32 kHz

- $<0.2 \mu \mathrm{A}$ typical standby current 


\subsection{LF353}

\section{FAIRCHILD}

SEMICONDUCTOR

\section{LF353}

\section{Dual Operational Amplifier (JFET)}

\section{Features}

- Internally trimmed offset voltage: $10 \mathrm{mV}$

- Low input bias current: 50pA

- Wide gain bandwidth: $4 \mathrm{MHz}$

- High slew rate: $13 \mathrm{~V} / \mu \mathrm{s}$

- High Input impedance: $10^{12} \Omega$

\section{Description}

The LF353 is a JFET input operational amplifier with an internally compensated input offset voltage. The JFET input device provides wide bandwidth, low input bias currents and offset currents.

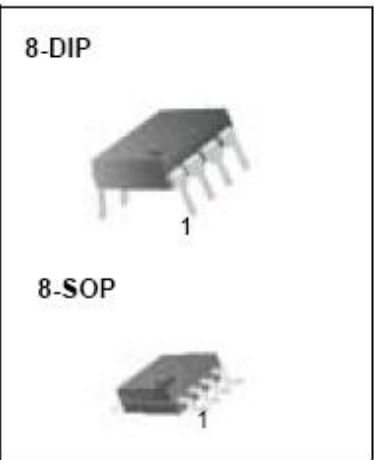

Internal Block Diagram

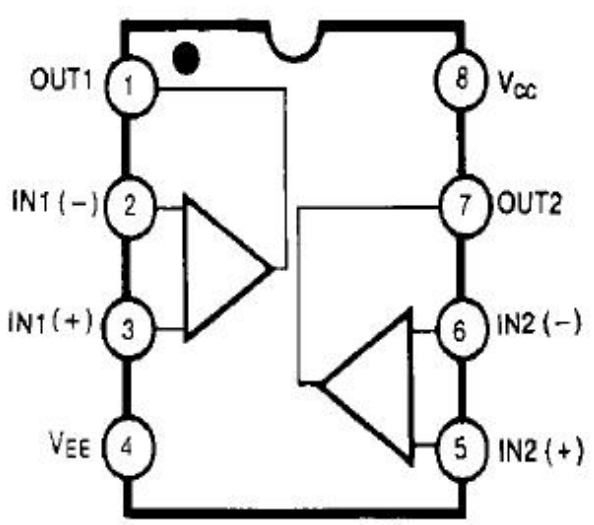




\section{Single/Dual Digital Potentiometer with SPI ${ }^{\mathrm{TM}}$ Interface}

\section{Features}

- 256 taps for each potentiometer

- Potentiometer values for $10 \mathrm{k} \Omega, 50 \mathrm{k} \Omega$ and $100 \mathrm{k} \Omega$

- Single and dual versions

- SPITM serial interface (mode 0,0 and 1,1 )

- \pm 1 LSB max INL \& DNL

- Low power CMOS technology

- 1 A A maximum supply current in static operation

- Multiple devices can be daisy-chained together (MCP42XXX only)

- Shutdown feature open circuits of all resistors for maximum power savings

- Hardware shutdown pin available on MCP42XXX only

- Single supply operation $(2.7 \mathrm{~V}-5.5 \mathrm{~V})$

- Industrial temperature range: $-40^{\circ} \mathrm{C}$ to $+85^{\circ} \mathrm{C}$

- Extended temperature range: $-40^{\circ} \mathrm{C}$ to $+125^{\circ} \mathrm{C}$

\section{Block Diagram}

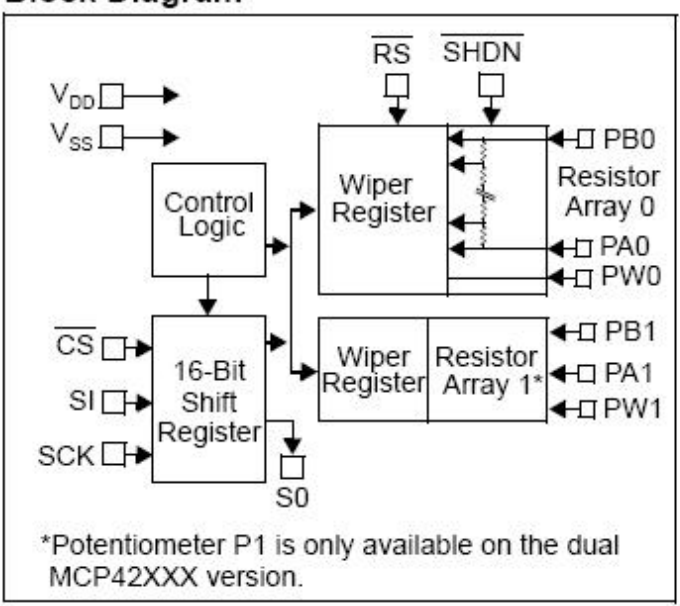

\section{Description}

The MCP41XXX and MCP42XXX devices are 256position, digital potentiometers available in $10 \mathrm{k} \Omega$, $50 \mathrm{k} \Omega$ and $100 \mathrm{k} \Omega$ resistance versions. The MCP41XXX is a single-channel device and is offered in an 8-pin PDIP or SOIC package. The MCP42XXX contains two independent channels in a 14-pin PDIP, SOIC or TSSOP package. The wiper position of the MCP41XXX/42XXX varies linearly and is controlled via an industry-standard SPI interface. The devices consume $<1 \mu \mathrm{A}$ during static operation. A software shutdown feature is provided that disconnects the " $A$ " terminal from the resistor stack and simultaneously connects the wiper to the "B" terminal. In addition, the dual MCP42XXX has a SHDN pin that performs the same function in hardware. During shutdown mode, the contents of the wiper register can be changed and the potentiometer returns from shutdown to the new value. The wiper is reset to the mid-scale position ( $80 \mathrm{~h}$ ) upon power-up. The $\overline{\mathrm{RS}}$ (reset) pin implements a hardware reset and also returns the wiper to mid-scale. The MCP42XXX SPI interface includes both the SI and SO pins, allowing daisy-chaining of multiple devices. Channel-to-channel resistance matching on the MCP42XXX varies by less than $1 \%$. These devices operate from a single $2.7-5.5 \mathrm{~V}$ supply and are specified over the extended and industrial temperature ranges.

\section{Package Types}

PDIPISOIC

\begin{tabular}{|c|c|c|c|c|}
\hline SL & 1 & & 8. & \\
\hline $\mathrm{K}$ & 2 & 웅 & 7 & \\
\hline & 3 & $\stackrel{\overrightarrow{\vec{x}}}{x}$ & 6 & \\
\hline$V_{S S}$ & 4 & $\underset{x}{x}$ & 5 & \\
\hline
\end{tabular}

PDIP/SOIC/TSSOP

\begin{tabular}{|c|c|c|c|c|}
\hline$\overline{\mathrm{CS}} \square$ & 1 & & 14 & $V_{D D}$ \\
\hline CK & 2 & & 13 & SO \\
\hline SI & 3 & $\bar{\Omega}$ & 12 & $\overline{\mathrm{SHDN}}$ \\
\hline$v_{S S}$ & 4 & $\frac{N}{N}$ & 11 & $\overline{R S}$ \\
\hline B1 & 5 & xx & 10 & $\square$ PBO \\
\hline $\mathrm{N} 1[$ & 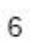 & & 9 & בPW0 \\
\hline PA1 & 7 & & 8 & $\beth \mathrm{PAO}$ \\
\hline
\end{tabular}




\subsection{MAX232}

19-4323; $\operatorname{Rev} 11 ; 2 / 03$

\section{MAXIMI \\ +5V-Powered, Multichannel RS-232 Drivers/Receivers}

\section{General Description}

The MAX220-MAX249 family of line drivers/receivers is intended for all EIATIA-232E and V.28/N.24 communications interfaces, particularly applications where $\pm 12 \mathrm{~V}$ is not available.

These parts are especially useful in battery-powered systems, since their low-power shutdown mode reduces power dissipation to less than $5 \mu \mathrm{W}$. The MAX225, MAX233, MAX235, and MAX245/MAX246/MAX247 use no external components and are recommended for applications where printed circuit board space is critical.

Applications

Portable Computers

Low-Power Modems

Interface Translation

Battery-Powered RS-232 Systems

Multidrop RS-232 Networks
Features

Superior to Bipolar

- Operate from Single +5V Power Supply ( $+5 \mathrm{~V}$ and $+12 \mathrm{~V}-$ MAX231/MAX239)

- Low-Power Receive Mode in Shutdown (MAX223/MAX242)

- Meet All EIA/TIA-232E and V.28 Specifications

- Multiple Drivers and Receivers

3-State Driver and Receiver Outputs

- Open-Line Detection (MAX243) Ordering Information

\begin{tabular}{|lll|}
\hline \multicolumn{1}{|c|}{ PART } & TEMP RANGE & PIN-PACKAGE \\
\hline MAX220CPE & $0^{\circ} \mathrm{C}$ to $+70^{\circ} \mathrm{C}$ & 16 Plastic DIP \\
\hline MAX220CSE & $0^{\circ} \mathrm{C}$ to $+70^{\circ} \mathrm{C}$ & 16 Narrow SO \\
\hline MAX220CWE & $0^{\circ} \mathrm{C}$ to $+70^{\circ} \mathrm{C}$ & 16 Wide SO \\
\hline MAX220C/D & $0^{\circ} \mathrm{C}$ to $+70^{\circ} \mathrm{C}$ & Dice \\
\hline MAX220EPE & $-40^{\circ} \mathrm{C}$ to $+85^{\circ} \mathrm{C}$ & 16 Plastic DIP \\
\hline MAX220ESE & $-40^{\circ} \mathrm{C}$ to $+85^{\circ} \mathrm{C}$ & 16 Narrow SO \\
\hline MAX220EWE & $-40^{\circ} \mathrm{C}$ to $+85^{\circ} \mathrm{C}$ & 16 Wide SO \\
\hline MAX220EJE & $-40^{\circ} \mathrm{C}$ to $+85^{\circ} \mathrm{C}$ & 16 CERDIP \\
\hline MAX220MJE & $-55^{\circ} \mathrm{C}$ to $+125^{\circ} \mathrm{C}$ & 16 CERDIP \\
\hline
\end{tabular}

Ordering Information continued at end of data sheet. ${ }^{*}$ Contact factory for dice specifications.

Selection Table

\begin{tabular}{|c|c|c|c|c|c|c|c|c|}
\hline $\begin{array}{l}\text { Part } \\
\text { Number }\end{array}$ & $\begin{array}{l}\text { Power } \\
\text { Supply } \\
\text { (V) }\end{array}$ & $\begin{array}{l}\text { No. of } \\
\text { RS-232 } \\
\text { Drivers/Rx }\end{array}$ & $\begin{array}{l}\text { No. of } \\
\text { Ext. Caps }\end{array}$ & $\begin{array}{l}\text { Nominal } \\
\text { Cap. Value } \\
(\mu \mathrm{F})\end{array}$ & $\begin{array}{l}\text { SHDN } \\
\text { \& Three- } \\
\text { State }\end{array}$ & $\begin{array}{l}\text { Rx } \\
\text { Active in } \\
\text { SHDN }\end{array}$ & $\begin{array}{l}\text { Data Rate } \\
\text { (kbps) }\end{array}$ & Features \\
\hline MAX220 & +5 & $2 / 2$ & 4 & 0.1 & No & - & 120 & Ultra-low-power, industry-standard pinout \\
\hline MAX222 & +5 & $2 / 2$ & 4 & 0.1 & Yes & - & 200 & Low-power shutdown \\
\hline MAX223 (MAX213) & +5 & $4 / 5$ & 4 & $1.0(0.1)$ & Yes & $\boldsymbol{v}$ & 120 & MAX241 and receivers active in shutdown \\
\hline MAX225 & +5 & $5 / 5$ & 0 & - & Yes & $\boldsymbol{v}$ & 120 & Available in SO \\
\hline $\operatorname{MAX} 230(\operatorname{MAX} 200)$ & +5 & $5 / 0$ & 4 & $1.0(0.1)$ & Yes & - & 120 & 5 drivers with shutdown \\
\hline MAX231 (MAX201) & $\begin{array}{l}+5 \text { and } \\
+7.5 \text { to }+13.2\end{array}$ & $2 / 2$ & 2 & $1.0(0.1)$ & No & - & 120 & $\begin{array}{l}\text { Standard }+5 /+12 \mathrm{~V} \text { or battery supplies; } \\
\text { same functions as MAX } 232\end{array}$ \\
\hline MAX232 (MAX202) & +5 & $2 / 2$ & 4 & $1.0(0.1)$ & No & - & $120(64)$ & Industry standard \\
\hline MAX232A & +5 & $2 / 2$ & 4 & 0.1 & No & - & 200 & Higher slew rate, small caps \\
\hline MAX233 (MAX203) & +5 & $2 / 2$ & 0 & - & No & - & 120 & No external caps \\
\hline MAX $233 \mathrm{~A}$ & +5 & $2 / 2$ & 0 & - & No & - & 200 & No external caps, high slew rate \\
\hline MAX234 (MAX204) & +5 & $4 / 0$ & 4 & $1.0(0.1)$ & No & - & 120 & Replaces 1488 \\
\hline MAX235 (MAX205) & +5 & $5 / 5$ & 0 & - & Yes & - & 120 & No external caps \\
\hline MAX236 (MAX206) & +5 & $4 / 3$ & 4 & $1.0(0.1)$ & Yes & - & 120 & Shutdown, three state \\
\hline MAX237 (MAX207) & +5 & $5 / 3$ & 4 & $1.0(0.1)$ & No & - & 120 & Complements IBM PC serial port \\
\hline MAX238 (MAX208) & +5 & $4 / 4$ & 4 & $1.0(0.1)$ & No & - & 120 & Replaces 1488 and 1489 \\
\hline MAX239 (MAX209) & $\begin{array}{l}+5 \text { and } \\
+7.5 \text { to }+13.2\end{array}$ & $3 / 5$ & 2 & $1.0(0.1)$ & No & - & 120 & $\begin{array}{l}\text { Standard }+5 /+12 \mathrm{~V} \text { or battery supplies; } \\
\text { single-package solution for IBMPC serial port }\end{array}$ \\
\hline $\operatorname{MAX} 240$ & +5 & $5 / 5$ & 4 & 1.0 & Yes & - & 120 & DIP or flatpack package \\
\hline MAX241 (MAX211) & +5 & $4 / 5$ & 4 & $1.0(0.1)$ & Yes & - & 120 & Complete IBM PC serial port \\
\hline MAX242 & +5 & $2 / 2$ & 4 & 0.1 & Yes & $\boldsymbol{v}$ & 200 & Separate shutdown and enable \\
\hline $\operatorname{MAX} 243$ & +5 & $2 / 2$ & 4 & 0.1 & No & - & 200 & Open-line detection simplifies cabling \\
\hline MAX244 & +5 & $8 / 10$ & 4 & 1.0 & No & - & 120 & High slew rate \\
\hline MAX245 & +5 & $8 / 10$ & 0 & - & Yes & $\boldsymbol{v}$ & 120 & High slew rate, int. caps, two shutdown modes \\
\hline MAX246 & +5 & $8 / 10$ & 0 & - & Yes & $\boldsymbol{v}$ & 120 & High slew rate, int. caps, three shutdown modes \\
\hline MAX247 & +5 & $8 / 9$ & 0 & - & Yes & $v$ & 120 & High slew rate, int. caps, nine operating modes \\
\hline MAX248 & +5 & $8 / 8$ & 4 & 1.0 & Yes & $\boldsymbol{v}$ & 120 & High slew rate, selective half-chip enables \\
\hline MAX249 & +5 & $6 / 10$ & 4 & 1.0 & Yes & $\boldsymbol{v}$ & 120 & Available in quad flatpack package \\
\hline
\end{tabular}

MPXV

Maxim Integrated Products

For pricing, delivery, and ordering information, please contact Maxim/Dallas Direct! at 1-888-629-4642, or visit Maxim's website at www.maxim-ic.com. 


\subsection{DISPLAY}

\section{Outline Dimension}
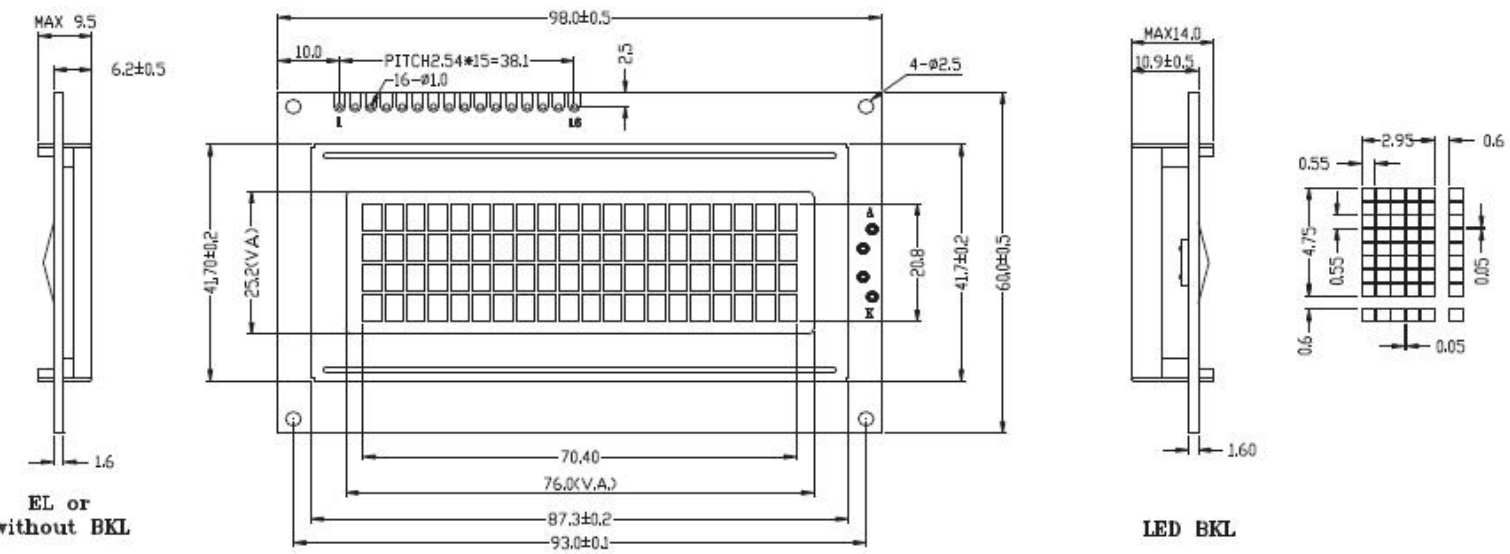

LED BKL

\section{Feature}

1. $5 X 8$ dots with cursor

2. Built-in controller (KS0066U or Equivalent)

3. $+5 \mathrm{~V}$ power supply (Also available for $+3.0 \mathrm{~V}$ )

4. 1/16 duty cycle

5. BKL to be driven by pin1, pin2, or pin15, pin 16, or $A, K$ 6. N.V.optional

\section{Interface pin connections}

\begin{tabular}{|c|l|l|}
\hline PIN NO & Symbol & \multicolumn{1}{|c|}{ Function } \\
\hline 1 & VSS & GND \\
\hline 2 & VDD & $+5 \mathrm{~V}$ \\
\hline 3 & V0 & Contrast adjustment \\
\hline 4 & RS & H/L Register select signal \\
\hline 5 & R/W & H/L Read/Write signal \\
\hline 6 & E & H/L Enable signal \\
\hline 7 & DB0 & H/L Data bus line \\
\hline 8 & DB1 & H/L Data bus line \\
\hline 9 & DB2 & H/L Data bus line \\
\hline 10 & DB3 & H/L Data bus line \\
\hline 11 & DB4 & H/L Data bus line \\
\hline 12 & DB5 & H/L Data bus line \\
\hline 13 & DB6 & H/L Data bus line \\
\hline 14 & DB7 & H/L Data bus line \\
\hline 15 & A & +4.2 V for BKL \\
\hline 16 & K & Power supply for BKL(0V) \\
\hline
\end{tabular}

Mechanical Data
\begin{tabular}{|l|c|c|}
\hline \multicolumn{1}{|c|}{ Item } & Standard & Unit \\
\hline Module dimension & $98.0 \times 60.0$ & $\mathrm{~mm}$ \\
\hline Viewing area & $78.0 \times 25.2$ & $\mathrm{~mm}$ \\
\hline Dot size & $0.55 \times 0.55$ & $\mathrm{~mm}$ \\
\hline Character size & $2.95 \times 4.75$ & $\mathrm{~mm}$ \\
\hline
\end{tabular}

\section{Absolute Maximum Rating}

\begin{tabular}{|c|c|c|c|c|c|}
\hline \multirow{2}{*}{ Item } & \multirow{2}{*}{ Symbol } & \multicolumn{3}{|c|}{ Standard } & \multirow{2}{*}{ Unit } \\
\cline { 3 - 5 } & & Min & Typ & Max & \\
\hline Power supply & $V D D-V S S$ & -0.3 & - & 5.5 & \multirow{2}{*}{$\mathrm{V}$} \\
\hline Input voltage & $V I$ & -0.3 & - & VDD & \\
\hline
\end{tabular}

\section{Electronical characteristics}

\begin{tabular}{|c|c|c|c|c|c|c|}
\hline \multirow{2}{*}{ Item } & \multirow{2}{*}{ Symbol } & \multirow{2}{*}{ Condition } & \multicolumn{3}{|c|}{ Standard } & \multirow{2}{*}{ Unit } \\
\hline & & & Min & Typ & $\operatorname{Max}$ & \\
\hline \multirow{2}{*}{ Input voltage } & \multirow{2}{*}{$V D D$} & $+5 \mathrm{~V}$ & 4.7 & 5.0 & 5.5 & $\mathrm{~V}$ \\
\hline & & $+3.3 \mathrm{~V}$ & 2.7 & 3.0 & 5.3 & V \\
\hline Supply current & 100 & $V D D=5 \mathrm{~V}$ & $\ldots$ & 3 & 5 & $\mathrm{~mA}$ \\
\hline \multirow{5}{*}{$\begin{array}{l}\text { Recommended } \\
\text { LCD driving voltage } \\
\text { for normal temp } \\
\text { version module }\end{array}$} & \multirow{5}{*}{ VDD-V0 } & $-20^{\circ} \mathrm{C}$ & $\ldots$ & $\ldots$ & $\ldots$ & \multirow{5}{*}{ V } \\
\hline & & $0^{\circ} \mathrm{C}$ & 4.7 & 5.0 & 5.5 & \\
\hline & & $25^{\circ} \mathrm{C}$ & 4.3 & 4.5 & 4.7 & \\
\hline & & $50^{\circ} \mathrm{C}$ & 4.1 & 4.3 & 4.5 & \\
\hline & & $70^{\circ} \mathrm{C}$ & $\ldots$ & $\ldots$ & $\ldots$ & \\
\hline LED forward voltage & VF & $25^{\circ} \mathrm{C}$ & $\ldots$ & 4.2 & 4.6 & $\mathrm{~V}$ \\
\hline LED forward current & IF & $25^{\circ} \mathrm{C}$ & $\ldots$ & 240 & - & $\mathrm{mA}$ \\
\hline $\begin{array}{l}\text { EL power supply } \\
\text { current }\end{array}$ & IEL & $\begin{array}{l}\mathrm{V}_{\mathrm{E}}=110 \mathrm{~V} \\
\mathrm{AC} 400 \mathrm{~Hz}\end{array}$ & - & - & - & $\mathrm{mA}$ \\
\hline
\end{tabular}

\section{Display character address code:}

$$
\text { Display position }
$$

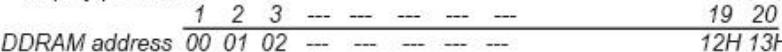

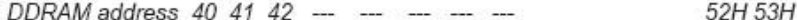

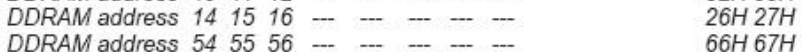




\section{LM2576/LM2576HV Series SIMPLE SWITCHER ${ }^{\circledR}$ 3A Step-Down Voltage Regulator}

\section{General Description}

The LM2576 series of regulators are monolithic integrated circuits that provide all the active functions for a step-down (buck) switching regulator, capable of driving $3 \mathrm{~A}$ load with excellent line and load regulation. These devices are available in fixed output voltages of $3.3 \mathrm{~V}, 5 \mathrm{~V}, 12 \mathrm{~V}, 15 \mathrm{~V}$, and an adjustable output version.

Requiring a minimum number of external components, these regulators are simple to use and include internal frequency compensation and a fixed-frequency oscillator.

The LM2576 series offers a high-efficiency replacement for popular three-terminal linear regulators. It substantially reduces the size of the heat sink, and in some cases no heat sink is required.

A standard series of inductors optimized for use with the LM2576 are available from several different manufacturers. This feature greatly simplifies the design of switch-mode power supplies.

Other features include a guaranteed $\pm 4 \%$ tolerance on output voltage within specified input voltages and output load conditions, and $\pm 10 \%$ on the oscillator frequency. External shutdown is included, featuring $50 \mu \mathrm{A}$ (typical) standby current. The output switch includes cycle-by-cycle current limiting, as well as thermal shutdown for full protection under fault conditions.

\section{Features}

- $3.3 \mathrm{~V}, 5 \mathrm{~V}, 12 \mathrm{~V}, 15 \mathrm{~V}$, and adjustable output versions

- Adjustable version output voltage range, $1.23 \mathrm{~V}$ to $37 \mathrm{~V}$ ( $57 \mathrm{~V}$ for $\mathrm{HV}$ version) $\pm 4 \%$ max over line and load conditions

- Guaranteed 3A output current

- Wide input voltage range, $40 \mathrm{~V}$ up to $60 \mathrm{~V}$ for HV version

- Requires only 4 external components

- $52 \mathrm{kHz}$ fixed frequency internal oscillator

- TTL shutdown capability, low power standby mode

- High efficiency

- Uses readily available standard inductors

- Thermal shutdown and current limit protection

- P+ Product Enhancement tested

\section{Applications}

- Simple high-efficiency step-down (buck) regulator

- Efficient pre-regulator for linear regulators

- On-card switching regulators

- Positive to negative converter (Buck-Boost)

Typical Application (Fixed Output Voltage Versions)

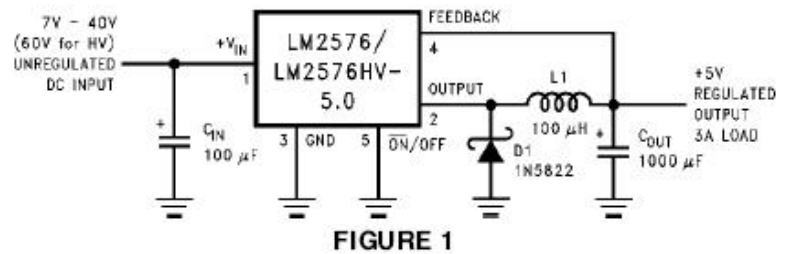

TLH/11476-1

\section{Block Diagram}

$3.3 \mathrm{VR} 2=1.7 \mathrm{k}$

$5 \mathrm{~V}, \mathrm{R} 2=3.1 \mathrm{k}$

$12 \mathrm{~V}, \mathrm{R} 2=8.84 \mathrm{k}$

$12 \mathrm{~V}, \mathrm{R} 2=8.84 \mathrm{k}$
$15 \mathrm{~V}, \mathrm{R} 2=11.3 \mathrm{k}$

For ADJ. Version

$\mathrm{R} 1=$ Open, $\mathrm{R} 2=0 \Omega$

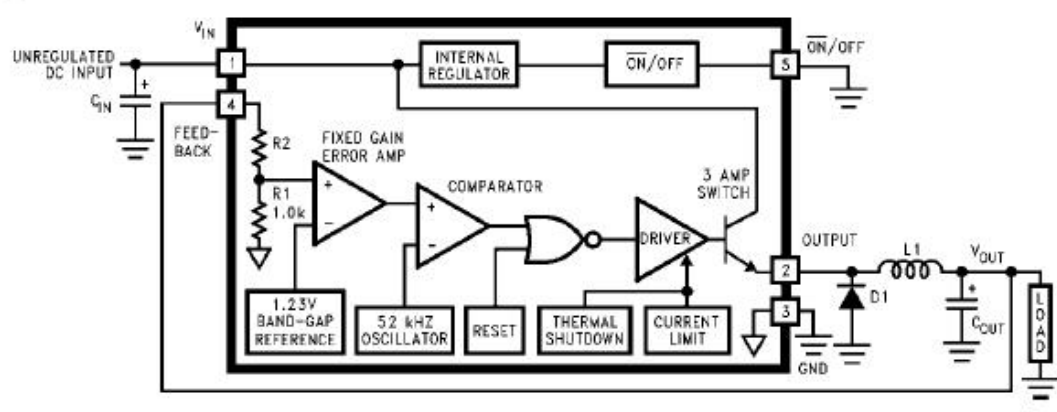

$\mathrm{TL} / \mathrm{H} / 11476-2$

Patent Pending

SIMPLE SWITCHER is a registered trademark of Natonal Semiconductor Corporaton 


\subsection{RAIZ DO PROGRAMA DO MICROCONTROLADOR}

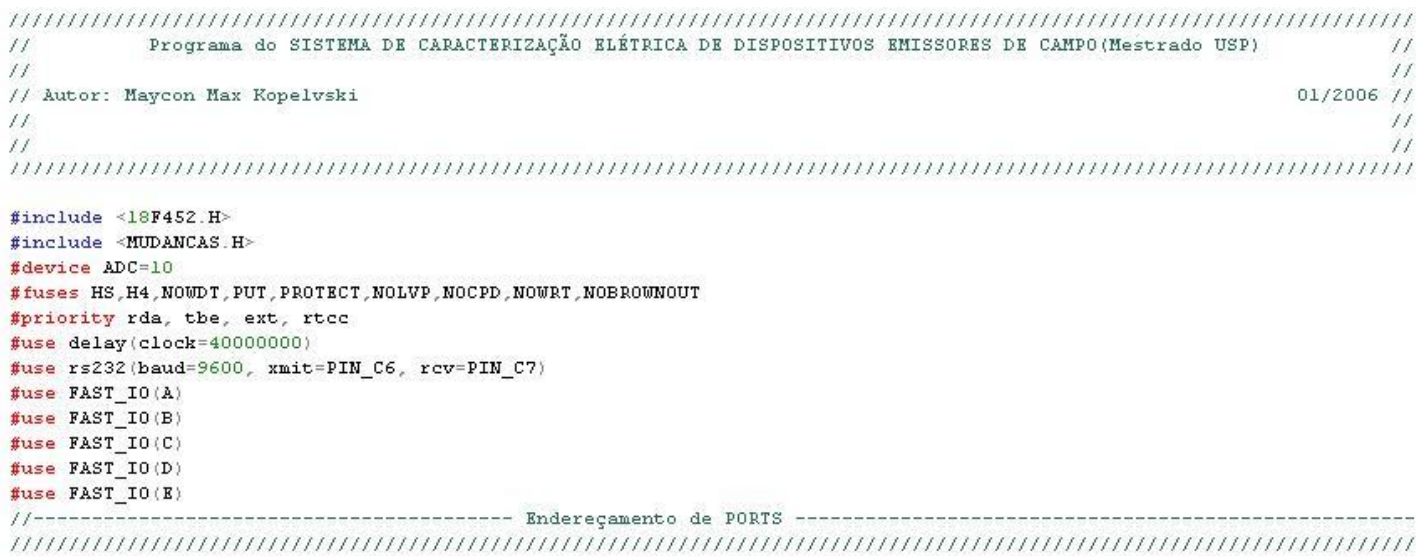

\#byte PortA $=0 \times \mathrm{F} 80$

\#byte PortB $=0 \times \mathrm{Fr}$

\#byte PortC $=0 \times \mathrm{F} 82$

\#byte PortD

thyte Port

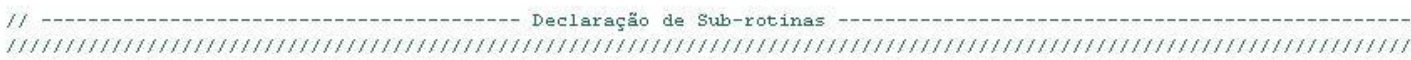

roid Rotinas_Iniciais (roid);

roid Limpa (roid)

roid Linha4 (char coluna)

roid Informacaol (roid)

void Confere (roid)

long int $\mathrm{Calc}_{-} \mathrm{Obj}$ (roid : :

roid Le Teclas (roid

void $\mathrm{Tecla} \mathrm{Dw}$ (roid)

roid Tecla Dupla (roid)

roid Beep_Curto (roid

void Calc_CRC (roid) :

char Numero (char Letra);

roid Beep_Longo (roid :
roid Send_oK (roid) :

roid Varre_Display (roid):

roid ConfereI (char valor) void Linha2 (char coluna) ?

roid Informacao 2 (roid):

roid Serial (roid)

roid Send_Valores (roid) :

roid Tecla Sd (roid)

roid Saida (roid)

roid Comuta_Esc (roid)

roid Confered (char valor

roid Informacao 3 (void)

oid Mensagem (roid) ?

void Linha3 (char coluna)

roid Separa (char Variavel

roid Inforenar Numero)

roid Calc_CRC50 (roid

char Recompoe (char

void Send_Brro (roid) macao 4 (roid)

char StrToChar (char caracter)

void Tecla Up (roid)

void Calc Vals (roid)

void Separal (long int Variavel)

void Clk_Dig (roid)

void $D i g$ Pot (char valor)

flot Decomo

float Becoupoes ichar

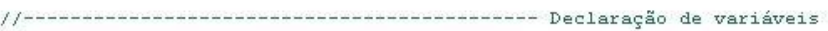

///////////////////////////////////////////////////////////////////////////////////////////////////////////////////

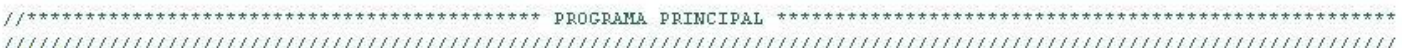
roid main (?

/ Inicialização

//Inicializa ports, periféricos, timers, etc.

//pinos

// Inicialização de pinos

//Vars

// Inicialização de Variáveis

//Flags

Inicialização de Flags

Inicializa(),

Confere (i)

Rotinas_Iniciais (?)

Thile $\{1\}\{" \ldots$ "... 


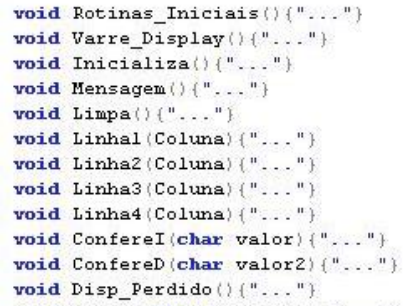

roid Separa (char Variavel) ("

roid Separa (Char Variave 1$)\{" \ldots$ ". $\}$

void Separaf (float Variavel) ("..."

char Recompoel (char v_l) ("...")

float Recompoes (char $\bar{v}_{-} 1, v_{-} 2, V_{-} 3, V_{-}$

chax RecompoesB (char $v_{-} 1, v_{-} 2, v_{-} 3$ )

char Numero (chax Letra) ("..."

char Letra (char Numero) " . "

void Le_Teclas () (" ". "

void Le Teclas (t) "..."

roid Tecla_sd ( ....')

roid Tecla_sela (..." ".."

void Tecla_SelB ()$\{" \ldots$

roid Tecla_Up () ("..."

roid Tecla_Dw ()$\{" \ldots$ "...

roid Tecla_Dupla() ("..."

roid Beep_Curto () ("...")

yoid Beep_Longo ( $)(" \ldots$ ". "

roid Pisca LED (char Tem LED , char T

void Saida ( ) "..."

roid Comuta_Bsc (\}$\{" \ldots$ "...

long int Calc obj ( $\{$ "..."

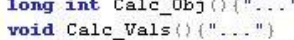

void Calc_Vals $\{\{" \ldots$ ".."

roid Informacaol ( ) \{"..." )

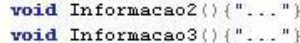

yoid Informacao 4 () ("..."

void Confere ( ) ("..."

void Send oK ( ) "..."

roid Send_Valores (\}, "..." \}

roid Send_Erro $\{($ "..."

roid Calc_CRC50 ( ) $\{" . . . "\}$

void Calc_CRC ()$\{" \ldots "\}$

short CRC_OK $\{("$...."

roid Serial ( $\{" \ldots$ "...

void Digpot (char valor) $\{"$ "..."

/Sub-rotina que possui as instruçōes de inicialização do Sistena

/Sub-rotina que possui as instruções de varredura do display 4 linas $\times 20$ caracteres

//Sub-rotina que possui as instruçōes de inicialização do display

/ Sub-rotina que possui as instruções de escrita no display

//Sub-rotina que possui as instruçöes para apagar as informaçöes mostradas no display

$/ /$ sub-rotina que possui as instruçóes de escrita na linha 1 do display

/ / Sub-rotina que possui as instruçóes de escrita na linha 2 do display

/Sub-rotina cque possui as instruçoses de escrita na linha 3 do display

//Sub-rotina que possui as instruções de escrita na linha 4 do display

/Sub-rotina que possui as instruções de transmissão de Instrução para o diaplsy

//Sub-rotina que possui as instruções de transmissão de Dados para o display

/Sub-rotina que possui as instruções de identificação de display perdido

Sub-rotina gue possui as instruçes para separar una variável tipo char

//Sub-rotina que possui as instruçőes para separar uma variável tipo long int

//Sub-rotina que possui as instruçöes para separar uma variável tipo float

//Sub-rotina que possui as instruçōes para recompor uma variável com 1 valor

5) \{"...") //Sub-rotina que possui as instruçöes para recompor uma variável com 5 valores (") //Sub-rotina que possui as instruçós para recompor uma variável com 3 valores $\left.4, v_{-} 5, v_{-} 6\right)(" . . . ") / /$ Sub-rotina que possui as instruçöes para recompor uma variável com 6 valores //Sub-rotina que possui as instruções de conversão de variável char para variável ASCII //Sub-rotina crue possui as instrucōes de conversão de variável ḋSCII para variável char //Sub-rotina que possui as instruções de leitura de teclas

//Sub-rotina que possui as açöes tomadas quando pressionada a tecla de Saída

//Sub-rotina que possui as açöes i tomadas cuando pressionada a tecla de Selecão

//Sub-rotina que possui as açōes B tomadas quando pressionada a tecla de seleção

//Sub-rotina que possui as ações B tomadas quando pressionada a tecla de Seleção
//Sub-rotina que possui as açóes tomadas quando pressionada a tecla direcional "Up"

//Sub-rotina que possui as ações tomadas quando pressionada a tecla direcional "Up"
//Sub-rotina que possui as ações tomadas quando pressionada a tecla direcional "Down"

//Sub-rotina que possui as ações tomadas quando pressionada a tecla direcional "DotNN"
//Sub-rotina que possui as as ações tomadas quando pressionada duas teclas ao mesmo tempo

//Sub-rotina que possui as instruçöes para um Beep Curto

//Sub-rotina que possui as instruçס̃es para um Beep Longo

em_LED2) \{"..." \} //Sub-rotina que possui as instruções para piscar o LED do Botão de Saída //Sub-rotina que possui as açöes relacionadas à saída da Fonte

//Sub-rotina cue possui as instruções de comutação de escala da Realimentação de Corrente / / Sub-rotina pue possui as instruções relacionadas às Realimentações

//Sub-rotina que possui as instruções relacionadas às Realimentações

//Sub-rotina que possui as instruçöes relacionadas ao gerenciamento da $1^{2}$ linha do display //Sub-rotina que possui as instruçōes relacionadas ao gerenciamento da $2^{2}$ linha do display //Sub-rotina que possui as instruçōes relacionadas ao gexenciamento da $3^{2}$ linha do display //Sub-rotina que possui as instruçöes relacionadas ao gerenciamento da $4^{2}$ linha do display //Sub-rotina que possui as instruçöes reacionadas à geração de caracteres //Sub-rotina que possui as instruções relacionadas à comunicação com o pC / Sub-rotina que possui as instruçoes relacionadas à comunicação com o DC / Sub-rotina gue possui as instrucines relacto

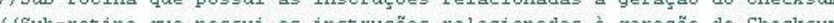
Tub-rotina que possui as intruçoes relacionadas a geraçå do checksuri //Sub-rotina que possui as instruçoes de checagem da palavra recebida do $/ /$ Sub-rotina que possui as instruçōes relacionadas à comunicação com o PC

//Sub-rotina que possui as instruções de controle do potenciômetro Digital

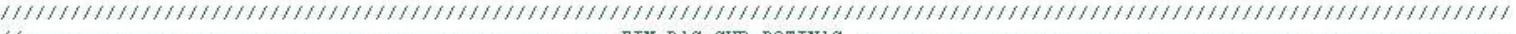

** INTBRRUPCÕOES *

\#int_rtec

void Tmrovf $(4)$ ("..."

\#int_timer2

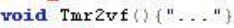

\#int_ext

void Int_exto ()$\{" \ldots$ "...

Hint $r d a$

roid int_rdal ()$\{" \ldots$ ".."

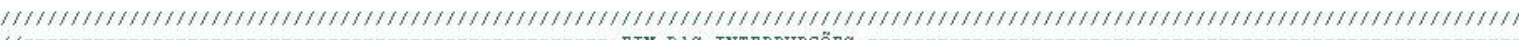

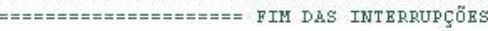


8.8. ESTRUTURA DO PROGRAMA HIGHVOLT CONTROLLER

PROGRAMA HighVolt Controller

$\rightarrow$ JANELA PRINCIPAL

A

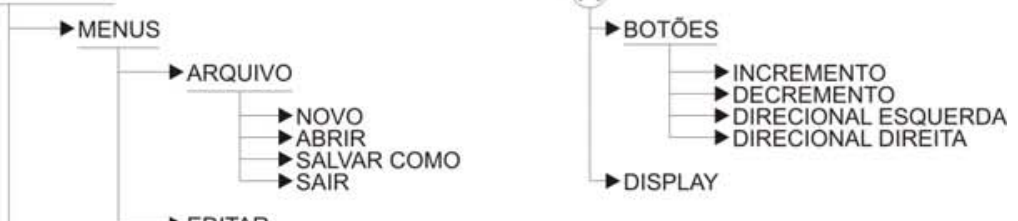

$\longrightarrow$ COMUNICAÇÃO

PESLIGADA

DESLIGADA

$\rightarrow$ SAIDA

$\longrightarrow$ LIGADA

DESLIGADA

$\rightarrow$ CONFIG COM

COM1

COM3

$\rightarrow D E B U G A R$

$\rightarrow$ SIM

$\rightarrow$ AJUDA

$\longrightarrow$ FAIXA DE PARÃMETROS

$\triangle$ BOTÓES

$\rightarrow$ NOVOS PARÂMETROS

- ABRIR PARÂMETROS

SALVAR PARAMETROS

- FAIXADE PARAMETROS

TALAR DADOS

SAIR

$\rightarrow$ PASTAS

$\rightarrow$ PARÂMETROS DE GRADE

$\longrightarrow$ BOTÖES

$\rightarrow$ FOCUS1

FOCUSS2

$\checkmark$ ZERO

$\rightarrow$ REPELER

-PARÂMETROS CONFIGURÁVEIS

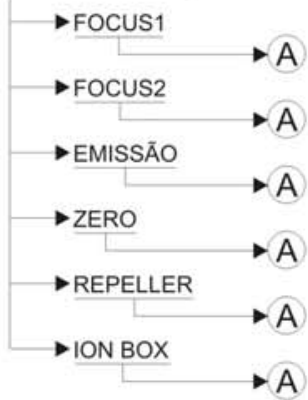

$\rightarrow$ PARÂMETROS DA FONTE

$\rightarrow$ BOTÖES

$\rightarrow$ CONTROLE MANUAL

$\rightarrow$ CONTROLE DE CORRENTE

$\rightarrow$ ESCALA DE CORRENTE

-PARÂMETROS CONFIGURÁVEIS

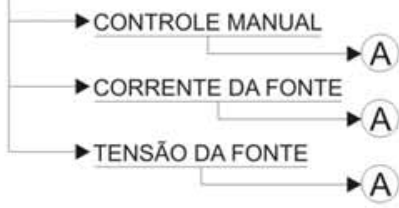




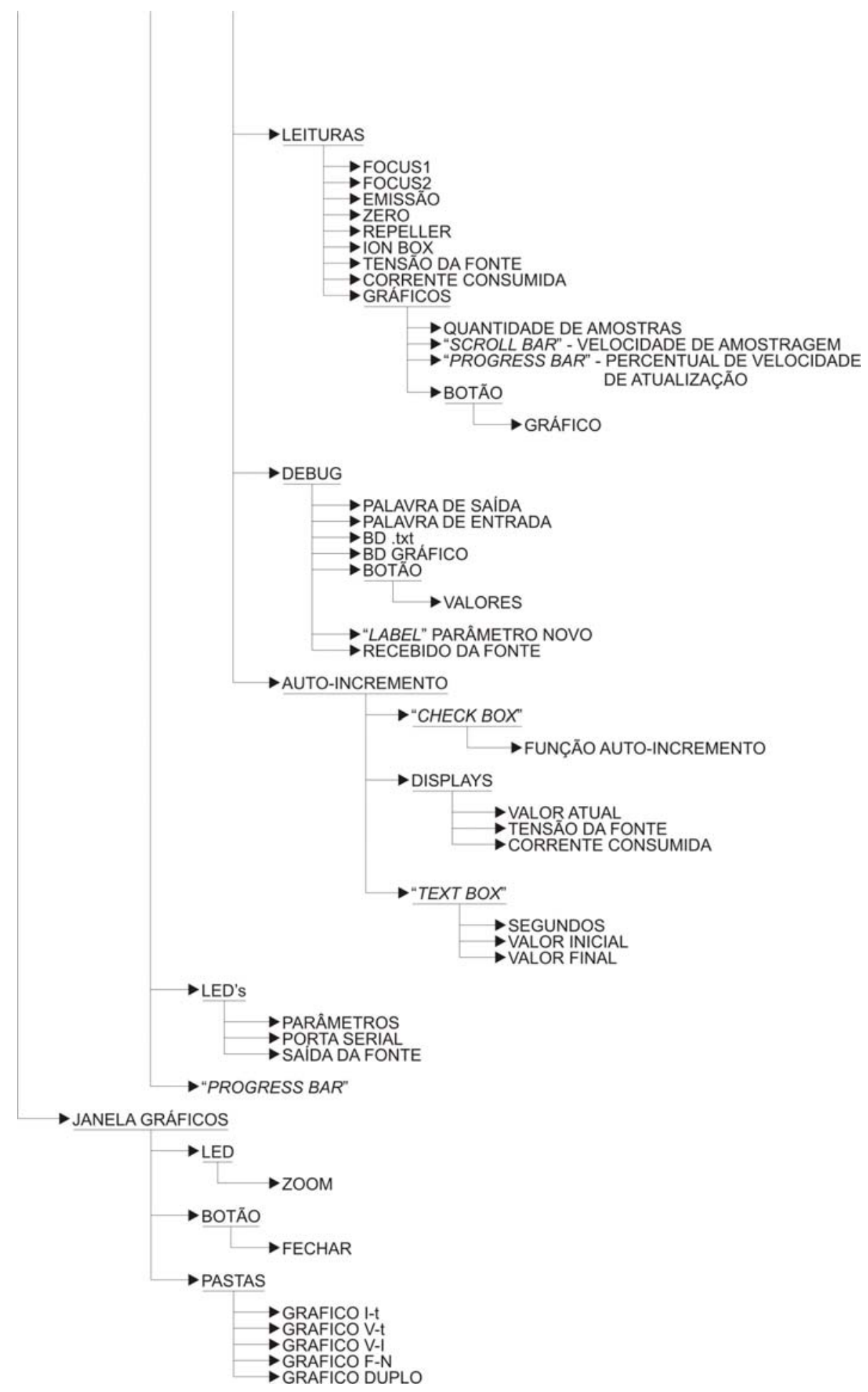


8.9. CALCULO DO CHECKSUM DO PACOTE DE DADOS

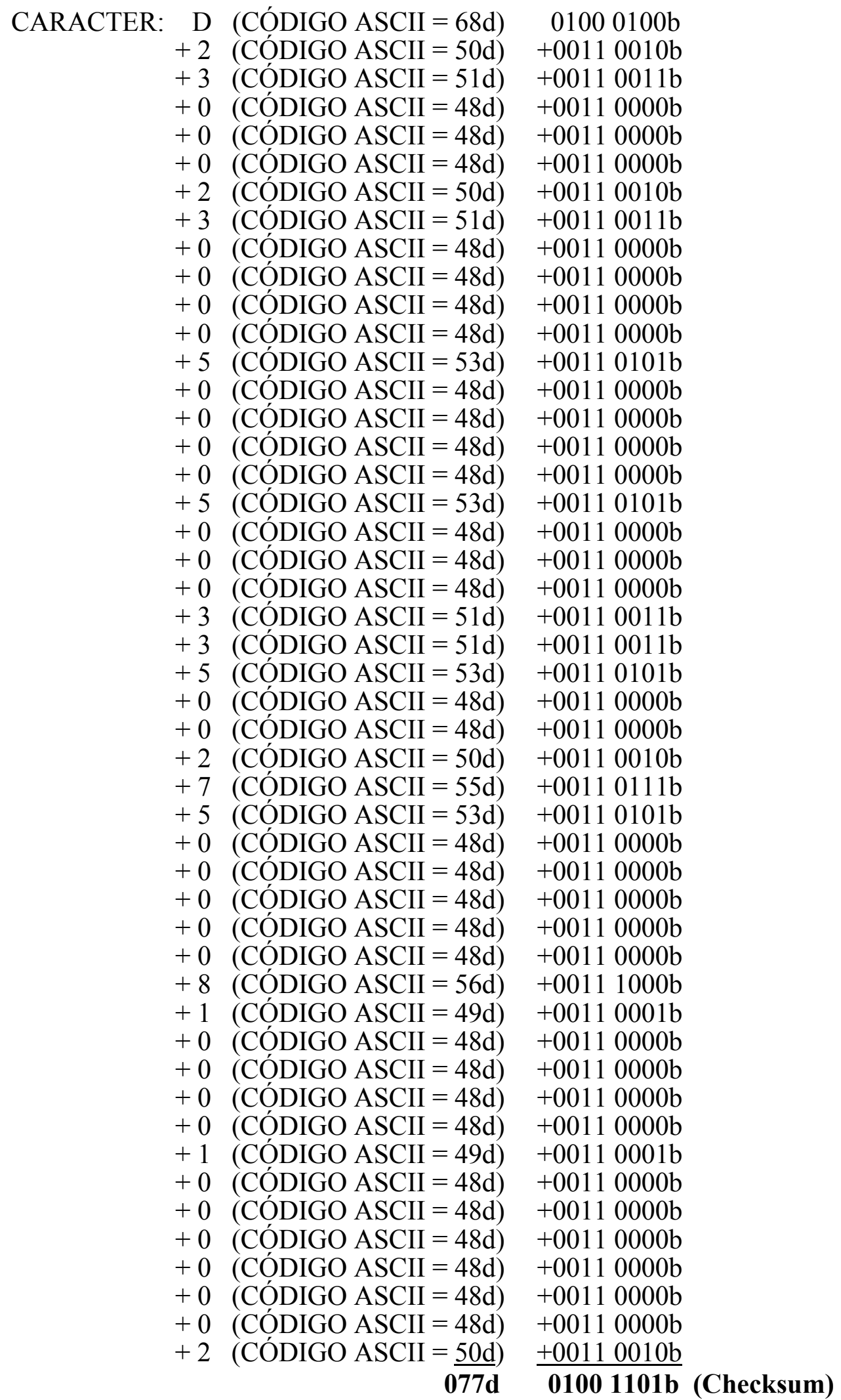

PALAVRA: D23000230000500005000335002750000081000010000002077 


\subsection{RAIZ DO PROGRAMA HIGHVOLT CONTROLLER}

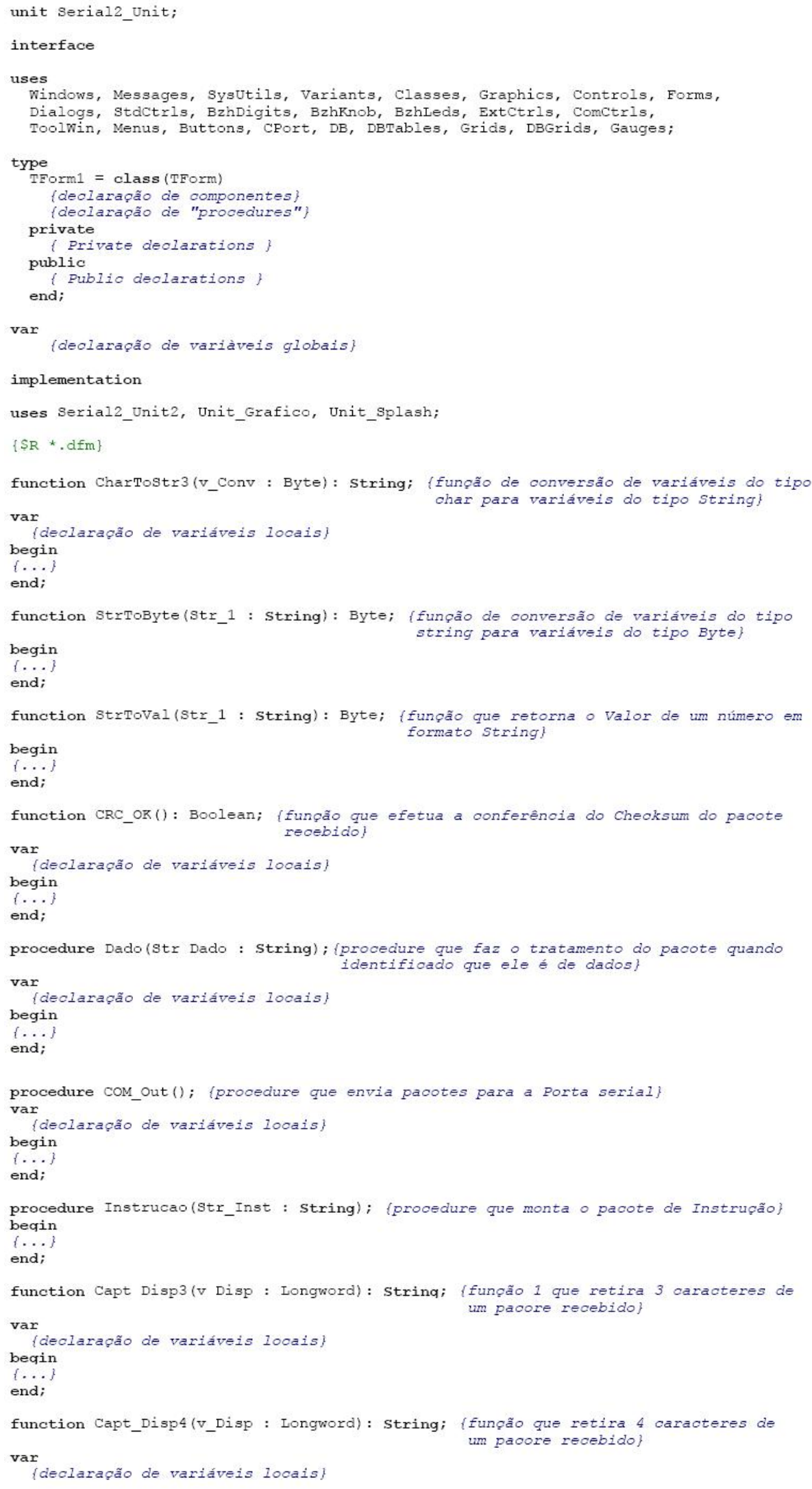


begin

(....

end

function Capt Disp3B(v Disp : Longword): String; ffunça 2 que retira 3 caracteres de um pacore recebido

var

(declaragão de variáveis looais\}

begin

end;

function LongToStr4(v Conv : Longword): String; ffunção de conversão de variáveis do tipo Long para variáveis do tipo string com 4 digitos\}

var

(declaragão de variáveis looais\}

begin

(....

end

function LongToStr5(v_Conv : Longword): String; ffunção de conversão de variáveis do tipo Long para variáveis do tipo string com 5

var digitos)

(declarạão de variáveis locais)

beqin

f...

end;

function LongToStr6(v_Conv : Longword): String; (fungão de conversão de variáveis do tipo Long para variáveis do tipo String com 6 digitos

(declarạão de variáveis locais\}

begin

$\{\ldots\}$

function DadoToLong(v Pos, $\mathrm{v}$ Tam : Integer): Longword; (função que retira uma variável Long de um pacore de dados recebido\}

var

\{declarậa de variáveis locais\}

begin

end;

procedure Atualiza_Palavra(); (procedure que faz a manupulą̧a do paoore de Saida\}

var

(declarạão de variáveis locais\}

begin

$\{.$. .

end;

function Capt Disp2(v Disp : Longword): String; (função que retira 2 caracteres de um pacore recebido\}

var declaragão de variáveis looais\}

begin

end;

procedure Atualiza Display(); (procedure que faz a manupulação dos Displays do

begin programa

begin

end

procedure Atualiza Botoes(); (procedure que faz a manupulação dos Botões do

begin

end;

procedure Fim_Prog(); (procedure que exeouta as rotinas quando o programa é fechado\}

begin

end;

procedure Reset_Prog(); (procedure que executa as rotinas de inicializạão quando o

programa é executado

begin

(....

function Dado PalavraOut(v1 Fos, v2 Tamanho : Integer):Integer; (função que retira uma

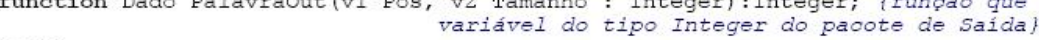

begin 
$\{\ldots\}$

function Dado_PalavraIn(v1_Pos, v2_Tamanho : Integer):Integer; (função que retira uma

begin

(...

variável do tipo Integer do paoote de Entrada

procedure TForm1.pg_2Show(Sender: TObject); (procedure que possui as rotinas que deven ser executadas sempre que à Fich

begin

"Parâmetros da Fonte" for visualizada)

end;

procedure TForml.Sairlclick(Sender: Tobject); (Procedure que é executada quando o $1^{\circ}$ botão "Sair" é aoionado

beqin
end;

procedure TForm1.FaixadosParmetros1Click(Sender: Tobject); (procedure executada quando o sub.menu "Ajuda => Faixa dos Parâmetros" é acionado)

begin
$\{. .$.

end;

procedure TForm1.FormClose(Sender: TObject; var Action: TCloseAction); (procedure executada sempre que o Programa é fechado)

begin

end;

procedure TForm1.Abrir1click(Sender: Tobject); (Procedure que é executada quando o botão

begin "Abrir" é acionado

end;

procedure TForml.Salvar1Click(Sender: TObject); (Procedure que é executada quando o botão "Salvar" é acionado)

begin

$\{\ldots\}$

end;

procedure TForm1.BitBtn4Clik (Sender: Tobject); (Procedure que é executada quando $\circ 2^{\circ}$ botão

becin "Sair" é acionado\}

end;

procedure TForm1.BitBtn2Click(Sender: TObject); (Procedure que é executada quando o $1^{\circ}$ botão "Abrir Parâmetros" é acionado\}

begin

end;

procedure TForml.BitBtn1Click(Sender: Tobject); (Procedure que é executada quando o botão

begin

(Procedure que é executada quando
"Salvar Parâmetros" é acionado\}

end;

procedure TForm1.BitBtn3Click(Sender: TObject); (Procedure que é executada quando o botão

begin "Faixa de Parâmetros" é acionado\}

end;

procedure TForm1.BitBtn5Click(Sender: TObject); Procedure que é executada quando o $2^{\circ}$ botão "Abrir Parâmetros" é acionado\}

begin

i...

end;

procedure TForm1.ComPort1RxChar(Sender: Tobject; Count: Integer); (procedure que é executada sempre que a Porta Serial recebe um pacote de Dados ou Instrupão

var

(declaragão de variáveis looais)

begin

end;

procedure TForm1.Novolclick(Sender: Tobject); (Procedure que é executada quando o botão "Novo" é acionado 


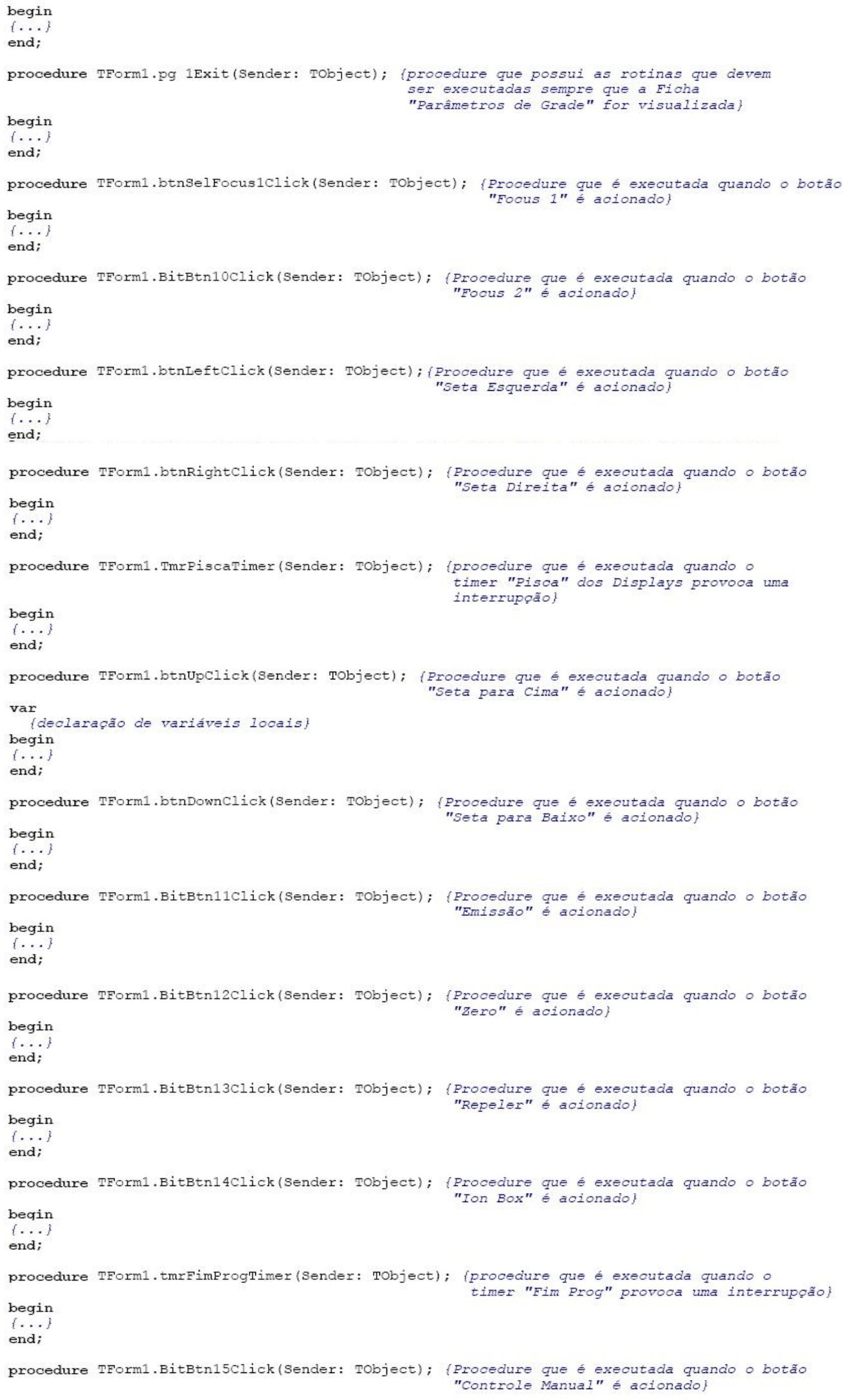




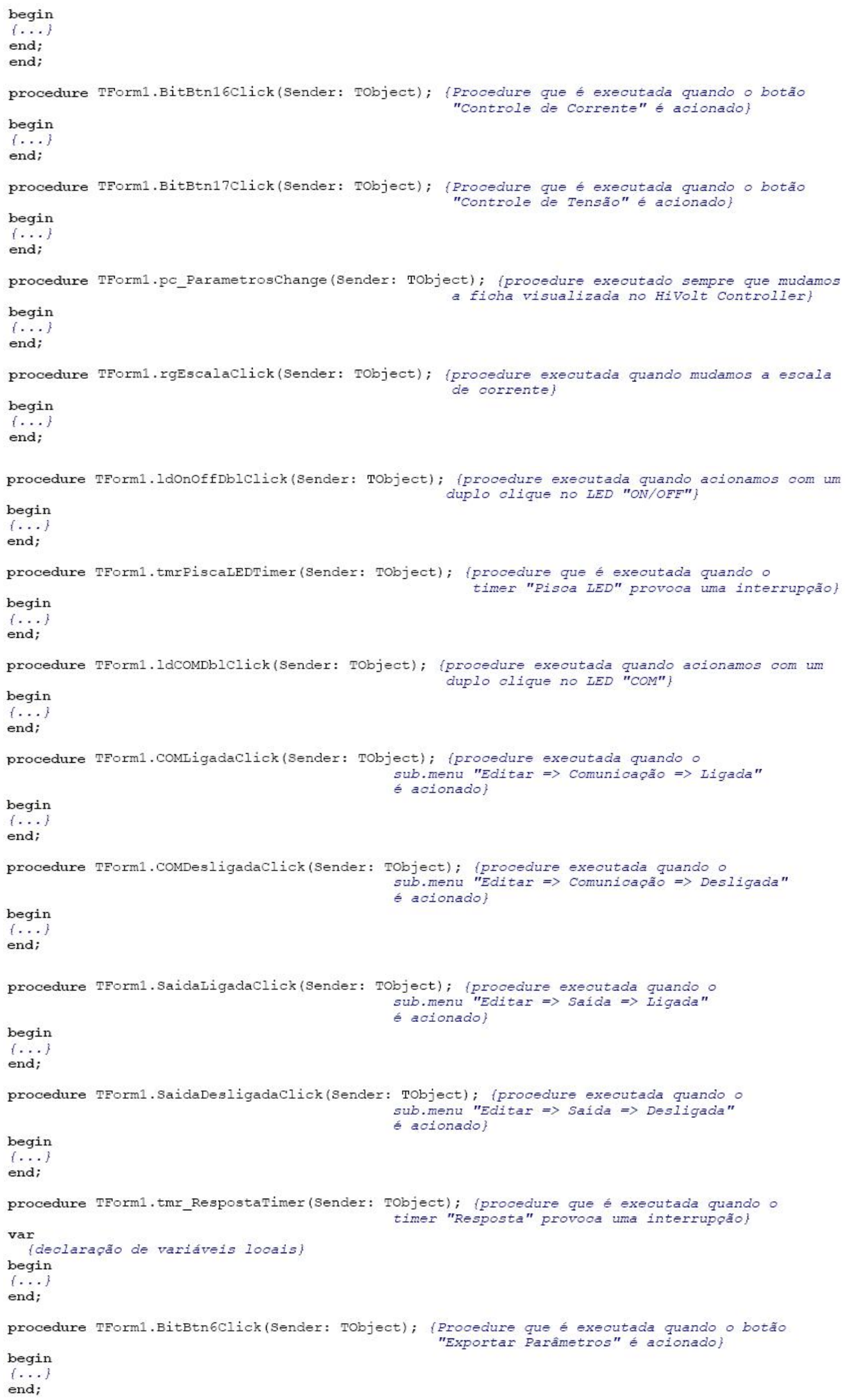




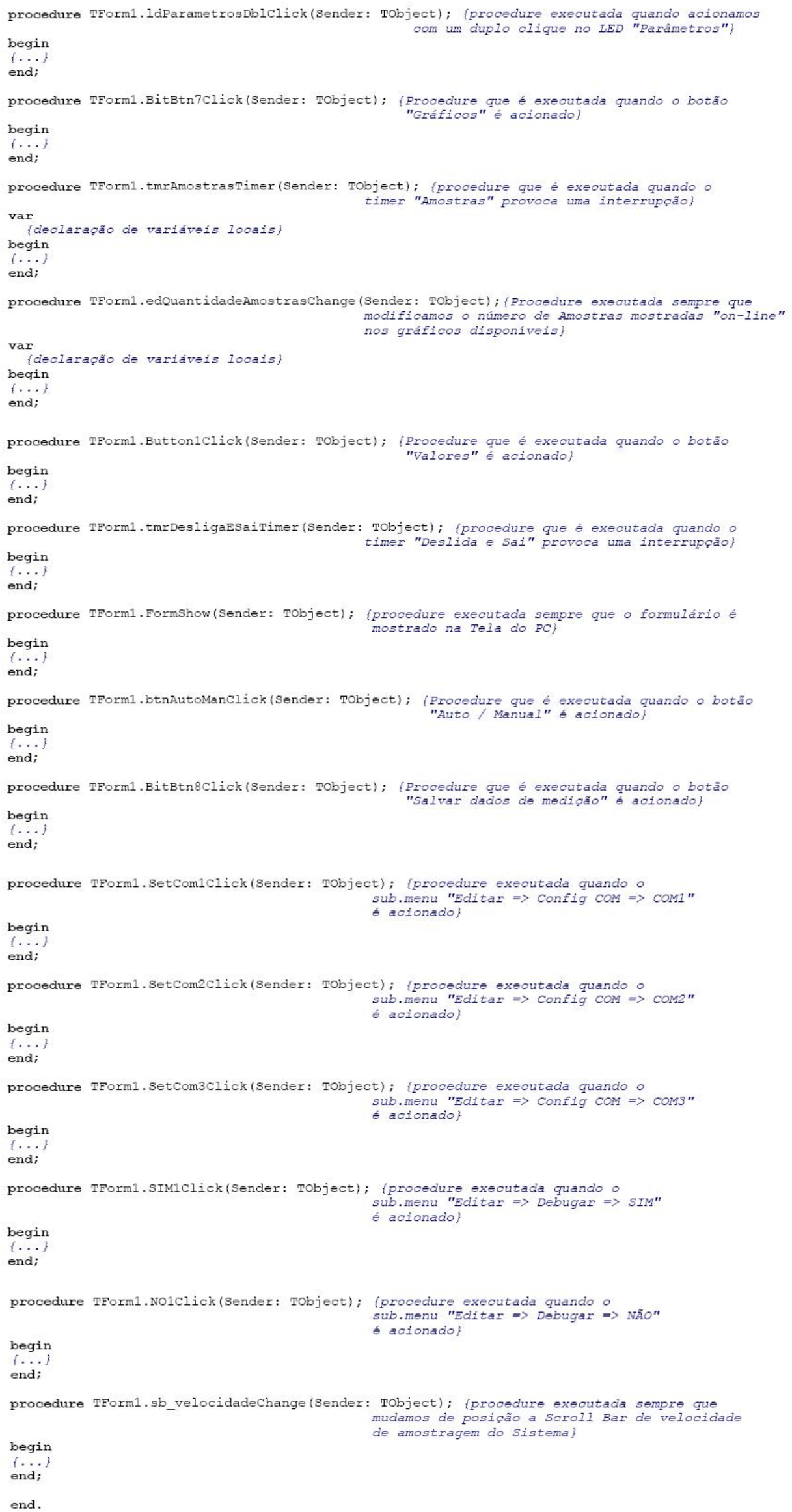

